\section{Stimuli Responsive Polymer/ Quantum Dot Hybrid Platforms Modified at the Nanoscale}

\title{
Invitation
}

It is my pleasure to invite you to

the public defense of my PhD thesis on

March 19th, 2010 at

$15: 00$ in room 4 of the

"Waaier" building of the University of Twente,

Enschede,

The Netherlands

At 14.45 , I will give

a brief introduction

to the content

of my thesis

\section{Oya Tagit}

o.tagit@tnw.utwente.nl

Paranimfen

Edit Kutnyanszky

Oya Tagit e.kutnyanszky@tnw.utwente.nl

Alexander van $\mathrm{Rhjin}$ a.c.w.vanrhijnr@tnw.utwente.n! 
STIMULI RESPONSIVE POLYMER/QUANTUM

DOT HYBRID PLATFORMS MODIFIEI AT

\author{
THE NANOSCALE
}


This research was financially supported by the MESA ${ }^{+}$Institute for Nanotechnology of the University of Twente (Strategic Research Orientation program Molecular Photonics) and the nanothechnology program NanoNed of the Dutch Ministry of Economic Affairs.

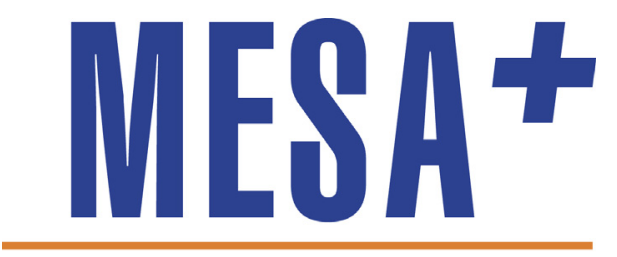

Institute for Nanotechnology

Stimuli Responsive Polymer/Quantum Dot Hybrid Platforms Modified at the Nanoscale

O. Tagit

$\mathrm{Ph}$. D Thesis

(C) Oya Tagit, Enschede, 2010

ISBN: 978-90-365-2982-2

Publisher: Ipskamp Drukkers B. V., Josink Maatweg 43, 7545 PS, Enschede, The Netherlends, http://www.ipskampdrukkers.nl

No part of this work may be reproduced by print, photocopy or any other means without the permission in writing of the author. 


\title{
STIMULI RESPONSIVE POLYMER/QUANTUM
}

\section{DOT HYBRII PLATFORMS MOIIFIED AT}

\section{THE NANOSCALE}

\author{
PROEFSCHRIFT
}

ter verkrijging van

de graad van doctor aan de Universiteit Twente,

op gezag van de rector magnificus,

prof. dr. H. Brinksma,

volgens besluit van het College voor Promoties

in het openbaar te verdedigen

op vrijdag 19 maart 2010 om 15.00 uur

door

Oya Tagit

geboren op 21 mei 1981

te Bursa, Turkije 
Dit proefschrift is goedgekeurd door:

Promotoren: Prof. dr. G.J. Vancso

Prof. dr. J.L. Herek

Assistent-promotor: dr. N. Tomczak 
This thesis is dedicated to my family 



\section{Table of Contents}

Chapter 1 General introduction

Chapter 2 Strategies towards fabrication of quantum dot/polymer assemblies 8

2.1. General introduction to quantum dots 9

$\begin{array}{ll}\text { 2.2. Optical properties of quantum dots } & 10\end{array}$

2.2.1. Surface modification strategies for quantum dots 13

2.2.2. $\operatorname{Poly}(N$-isopropylacryl amide) as encaging agent for 14 quantum dots

2.3. Routes for designing polymer/quantum dot hybrid assemblies $\quad 18$

2.3.1. Layer-by-layer electrostatic assembly approach $\quad 18$

2.3.2. Loading quantum dots within polymeric matrices during the 21 polymer synthesis

2.3.3. Grafting approaches $\quad 25$

2.3.4. In situ synthesis of quantum dots within polymeric matrices $\quad 27$

2.4. Conclusions 31

2.5. References 31

Chapter 3 Characterization methods of quantum dots and quantum dot / 37 polymer assemblies

3.1. Introduction 38

3.2. Microscopy techniques $\quad 38$

3.2.1. Atomic force microscopy 38

3.2.2. Confocal microscopy 42

3.2.3. Combination of different microscopy techniques 43

3.3. Spectroscopy techniques 44

3.3.1. Time correlated single photon counting (TCSPC) 45

3.3.2. Fluorescence correlation spectroscopy (FCS) 45

3.4. References 47

Chapter 4 Probing the morphology and nano-scale mechanics of single poly(N- 50 isopropylacrylamide) microparticles across the lower critical solution temperature by atomic force microscopy 
4.1. Introduction $\quad 51$

4.2. Experimental section $\quad 52$

4.3. Results and discussion $\quad 54$

4.4. Conclusions 64

4.5. References 65

Chapter 5 Temperature modulated quenching of quantum dots covalently $\quad 68$ coupled to chain ends of poly( $\mathrm{N}$-isopropyl acrylamide) brushes on gold

5.1. Introduction $\quad 69$

$\begin{array}{ll}\text { 5.2. Experimental section } & 70\end{array}$

5.3. Results and discussion $\quad 71$

5.4. Conclusions 78

$\begin{array}{ll}\text { 5.5. References } & 78\end{array}$

$\begin{array}{lll}\text { Chapter } 6 & \text { Thermoresponsive quantum dot/PNIPAM assemblies } & 81\end{array}$

6.1. Introduction $\quad 82$

6.2. Experimental section $\quad 83$

6.3. Results and discussion $\quad 85$

6.4. Conclusions 93

6.5. References 94

Chapter 7 Optical characterization of thermo-responsive polymer-quantum 97 dot nanoparticles

$\begin{array}{ll}\text { 7.1. Introduction } & 98\end{array}$

7.2. Experimental section 100

7.3. Results and discussion 101

$\begin{array}{ll}\text { 7.4. Conclusions } & 108\end{array}$

$\begin{array}{ll}\text { 7.5. References } & 109\end{array}$

Chapter 8 Applications of quantum dots in bio-medicine: opportunities and 111 risks

8.1. Introduction 112

8.2. Applications of quantum dots in biology and medicine 113

8.2.1. Applications of quantum dots as in vitro fluorescent labels $\quad 114$ 
8.2.3. Applications of quantum dots in photodynamic therapy of cancer

8.3. QD toxicity

8.4. Conclusions

Summary

Samenvatting

Pêşgotîn bi Kurtayî

Acknowledgements

135

About the author

138 



\section{List of Abbreviations}

QD quantum dot

TOPO trioctylphosphine oxide

PNIPAM poly( $N$-isopropylacryl amide)

LCST lower critical solution temperature

VPTT volume phase transition temperature

PL photoluminescence

LbL layer-by-layer

AFM atomic force microscopy

TCSPC time correlated single photon counting

FCS fluorescence correlation spectroscopy

BIS $N, N^{\prime}$-methylenebisacrylamide

KPS potassium persulfate

MAA mercaptoacetic acid

DTCA dithiodiundecane-11,1-diylbis $\{4[([($ diethylamino $)$ carbonothioyl $]$ thioethyl)phenyl]carbamate

DDS 1,2-dioctadecyldisulfane

ODT octadecane-1-thiol

TEMPO 2,2,6,6-tetramethylpiperidine-1-oxyl

EDC 1-ethyl-3-[3-dimethylaminopropyl]carbodiimide

NHS N-hydroxysuccinimide

DIPEA $N, N$-diisopropylethylamine

FWHM full width at half maximum 



\section{Chapter 1}

General introduction 

Nanotechnology aims at designing and creating functional materials, structures, devices and systems through the direct control of matter on the nanometer scale and at exploitation of novel phenomena and properties on this length scale, which is defined as being smaller than $100 \mathrm{~nm}$ [1]. Obtaining a fundamental understanding of the optical, electrical, magnetic and mechanical properties of nanostructures as well as controlled manipulation of these materials into complex, functional architectures requires multidisciplinary effort and cross-fertilization among different disciplines.

Nanoscale engineering of materials enables controlled alteration, dynamical manipulation, and molecular functionalization of materials' properties, and potentially creates entirely new properties, which are inaccessible otherwise.

Complementary to traditional 'Top-Down' material processing approaches, nanotechnology have enabled 'Bottom-Up' processes, inspired by nature, involving building up materials from the molecular levels to nano/macrometer sized structures [2]. For the 'Bottom-Up' approach, colloidal systems with diameters smaller than $50 \mathrm{~nm}$ are generally of interest [2]. These nano-sized particles, owing to their dimensions, have become a 'hot' topic in colloid and materials science [3] due to their unique electronic, optical, photoresponsive and catalytic properties [4], as well as to their applications in nanotechnology from biological labels to lasers and LEDs [5-14].

Such particles display different properties than in the bulk, and are usually dependent on the shape and size of the individual particles, as well as on the distance between those particles. Among the nano-particles semiconductor nanocrystals, Quantum Dots, (QDs) have become of considerable scientific and technological interest due to the opportunity they offer in the quantum confined regime [15-20].

QDs have recently entered the realm of biology owing to their advantages as biological probes including their nanoscale size (similar to biomolecules (Figure 1.1)), high quantum yield and molar extinction coefficients, versatility in surface modification, broad excitation spectra (for multicolor imaging) and narrow band emission (Figure 1.2), and tunable optical properties [21-24]. 


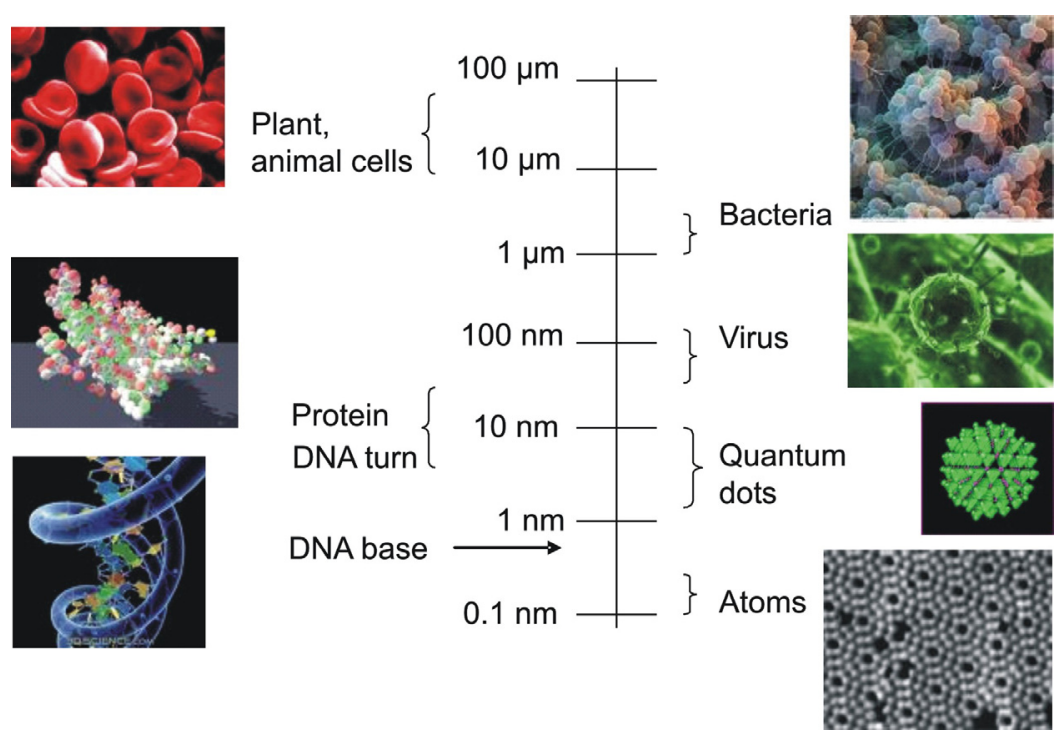

Figure 1.1. Length scales showing the comparable sizes of biomolecules and QDs.

While QDs easily mix in various solvents, utilization of their functionality in practical integrated photonic systems or in physiological environments requires QDs being distributed in a robust and highly functional matrix. In this respect organic polymers are of great potential as hosts for QDs $[25,26]$. Polymers are usually transparent in a wide spectral range and can be easily processed, providing a flexible platform for devices based on optical properties of QDs [27].

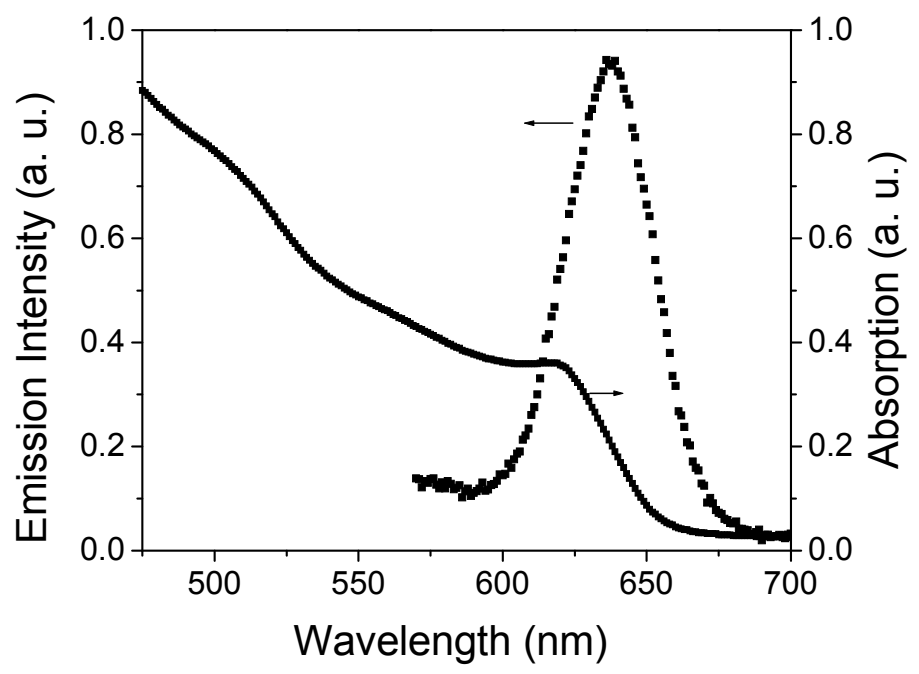

Figure 1.2. Normalized absorption and emission spectra of TOPO-coated CdSe/ZnS QDs in chloroform solution. 
Encaging QDs within polymer matrices not only enables the control over optical and spectroscopic properties of QDs but also introduces a strong resistance to chemical and photodegradation [28] as well as an enhanced compatibility to biological environment. In this respect, these colloidal fluorescent hybrid materials hold great promise for use as fluorescent probes in targeting, labeling and imaging applications [29-33].

Stimuli-responsive polymers, which undergo large physical changes upon small variations of external stimuli, such as temperature [34], $\mathrm{pH}$ [35], electric field [36], ionic strength, solvent composition [37], have attracted a great deal of attention as QD-encaging matrices. Using these 'smart' polymers for encaging QDs, one could prepare biosensor devices that would be switched on and off in response to the external stimuli [38]. Poly $(N-$ isopropylacrylamide) (PNIPAM) is one of the best studied temperature-responsive polymers, which exhibits a lower critical solution temperature (LCST) at $\sim 32^{\circ} \mathrm{C}$ [39]. The hybrid devices possessing optical properties of QDs with temperature-responsive properties of PNIPAM will be capable of sensing and detecting the state of biological systems and living organisms optically, electrically and magnetically at the single molecule level.

The work presented in this thesis covers synthesis and characterization of $\mathrm{CdSe} / \mathrm{ZnS}$ core/shell QDs, and synthesis and characterization of temperature-responsive polymer matrices made of PNIPAM, as carriers of QDs. Fabrication of QD/temperature responsive polymer assemblies are presented with potential applications as sensing devices to be used in bio-nanotechnology.

Chapter 2 provides a basic background in the optical properties of QDs and a literature review regarding the recent developments in combining stimuli responsive polymers with QDs via different approaches, including layer-by-layer deposition, macromolecular grafting and in situ QD synthesis within polymeric matrices. Particular attention is paid to the assemblies of QDs with the temperature-responsive polymer PNIPAM.

Chapter 3 describes the basics of some of the microscopy and spectroscopy techniques used for the characterization of QDs down to the single molecule level. In addition to atomic force microscopy and confocal optical microscopy, combined microscopy techniques for studying QDs and QD-bearing systems are explained. Spectroscopy techniques that are mentioned in this chapter include time correlated single photon counting and fluorescence correlation spectroscopy.

Chapter 4 presents the study of the morphology and nano-mechanical properties of individual, isolated, PNIPAM microgel particles at the silicon/air, and silicon/water 
interfaces, below and above the PNIPAM volume phase transition temperature (VPTT) using atomic force microscopy. The force-indentation measurements performed in air and in water below and above the VPTT of PNIPAM were used for the determination of the modulus of the PNIPAM spheres.

In Chapter 5 a thermo-responsive polymer/quantum dot platform based on PNIPAM brushes 'grafted from' a gold substrate and QDs covalently attached to the PNIPAM layer is presented. The influence of PNIPAM chain collapse above its lower critical solution temperature (LCST) on the QD luminescence is discussed.

Chapter 6 describes the synthesis of maleic anhydride-based copolymers with grafted PNIPAM chains. The resulting amphiphilic polymers are used as coatings for hydrophobic QDs. Colloidal and optical characterization of QDs coated with the novel coatings in aqueous solutions as a function of temperature is presented.

Chapter 7 includes a detailed analysis of the colloidal and optical properties of QD/PNIPAM assemblies presented in Chapter 6. Fluorescence correlation spectroscopy and time correlated single photon counting measurements carried out at temperatures below and above the LCST of PNIPAM are reported.

In Chapter 8 applications of QDs in in vitro labeling, in vivo detection and photo dynamic therapy are reviewed. Potential risks of toxicity of QDs in biological systems are presented with suggestions for making QDs non-toxic to biological systems in an environment-friendly fashion.

\section{References}

1- Vancso, G. J., Eur Polym J 2004, 40: p. 883

2- $\quad$ Sugunan, A.; Dutta, J., J Phys Sci Ide 2004, 4: p. 50

3- $\quad$ Schneider, G.; Decher, G. Nano Lett, 2004, 4: p. 1833

4- Shon, Y. S. Dekker Encyclopedia of Nanoscience \& Nanotechnology 2004, Marcel Dekker, New York

5- $\quad$ Chan, W.C.W.; Maxwell, D. J.; Gao, X.; Bailey, R. E.; Han, M.; Nie, S. Curr. Opin. Biotechnol. 2002, 13: p. 40

6- $\quad$ Seydel, C., Science 2003, 300: p. 80

7- Jovin, T., Nat. Biotechnol. 2004, 21: p. 32 
8- Jiang, W.; Papa, E.; Fischer, H.; Mardyani, S.; Chan, W.C.W. Trends Biotechnol. 2004, 22: p. 607

9- $\quad$ Chan, W. C.; Nie, S. Science 1998, 281: p. 2016

10- Wuister, S. F.; Donega, C. M.; Meijerink, A. J. Phys. Chem. B 2004, 108: p. 17393

11- Bruchez, M., Jr.; Moronne, M.; Gin, P.; Weiss, S.; Alivisatos, A.P. Science 1998, 281: p. 2013

12- Dubertret, B.; Skourides, P.; Norris, D. J.; Noireaux, V.; Brivanlou, A. H.; Libchaber, A. Science 2002, 298: p. 1759

13- Klimov, V.I.; Mikhailovsky, A.A.; Xu, S.; Malko, A.; Hollingsworth, J.A.; Leatherdale, C. A. Eisler, H. Bawendi, M. G. Science 2000, 290: p. 314

14- Coe, S. Woo, W.-K. Bawendi, M. G. Bulovic, V. Nature 2002, 420: p. 800

15- Alivisatos A. P. Science 1996 271: p. 933

16- Huynh, W. U.; Dittmer, J. J.; Alivisatos, A. P. 2002 Science 295: p. 2425

17- Klein, D.; Roth, R.; Lim, A.K.L.; Alivisatos, A.P.; McEuen, P.L., 1997 Nature 389: p. 699

18- $\quad$ Eychmuller, A., 2000 J. Phys. Chem. B 104: p. 6514

19- Trindade, T.; O’Brien, P.; Pickett, N.L., 2001 Chem. Mater. 13: p. 3843

20- Trindade, T., 2003 NanoscaleMaterials ed L.M. Liz-Marz'an and P.V. Kamat (Dordrecht: Kluwer-Academic)

21- Nehilla, B. J.; Vu, T. Q.; Desai, A. T. J. Phys. Chem. B 2005, 109: p. 20724

22- Medintz, I. L.; Uyeda, H. T.; Goldman, E. R.; Mattoussi, H. Nature Mat. 2005, 4: p. 435

23- Ho, Y. P.; Kung, M. C.; Yang, S.; Wang, T. H. Nano Lett. 2005, 9: p. 1963

24- Nagasaki, Y.; Ishii, T.; Sunaga, Y.; Watanabe, Y.; Otsuka, H.; Kataoka, K. Langmuir 2004, 20: p. 6396

25- Tessler, N.; Mededev, V.; Kazes, M.; Kan, S. H.; Banin, U.; Science 2002, 295: p. 1506

26- Bakueva, L.; Musikhin, S.; Hines, M. A.; Chang, T. W. F.; Tzolov, M.; Scholes, G. D.; Sargent, E. H. Appl. Phys. Lett. 2003, 82: p. 2895

27- Olsson, Y. K.; Chen, G.; Rapaport, R.; Fuchs, D. T.; Sundar, V. C.; Steckel, J. S.; Bawendi, M. G.; Aharoni, A.; Banin, U. Appl. Phys. Lett. 2004, 85: p. 4469

28- Medintz, I. L.; Sapsford, K. E.; Konnert, J. H.; Chatterji, A.; Lin, T.; Johnson, J. H.; Mattoussi, H. Langmuir, 2005, 21: p. 5501 
29- Jaiswal J. K.; Mattoussi, H.; Mauro, J. M.; Simon, S. M. Nat. Biotechnol. 2003, 21: p. 47

30- Jaiswal, J. K.; Simon, S. M. Trends Cell. Biol. 2004, 14: p. 497

31- Ballou, B.; Lagerholm, B. C.; Ernst, L. A.; Bruschez, M. P.; Waggoner, A. S. Bioconjugate Chem. 2004, 15: p. 79

32- Dahan, M.; Le'vi, S.; Luccardini, C.; Rostaing, P.; Riveau, B.; Triller, A. Science 2003, 302: p. 442

33- Chan, W. C. W.; Nie, S. M. Science 1998, 281: p. 2016

34- Xulu, P. M.; Filipsei, G.; Zrinyi, M. Macromolecules 2000, 33: p. 1716

35- Tanaka, T.; Fillmore, D.; Sun, S.-T.; Nishio, I. Phys. Rev. Lett.1980, 45: p.1636

36- Tanaka, T.; Nishio, I.; Shun, S.-T.; Ueno-Nishio, S. Science 1982, 218: p. 467

37- Pagonis, K.; Bokias, G. Polymer 2004, 45: p. 2149

38- $\quad$ Tomczak, N.; Janczewski, D.; Han, M.-Y.; Vancso, G.J., Prog Polym Sci, 2009. 34: p. 393.

39- Schild, H. G.; Tirrell, D. A., Langmuir 1991, 7: p. 665 


\section{Chapter 2}

\section{Strategies towards fabrication of quantum dot/polymer assemblies"}

This chapter provides a basic background in the optical properties of QDs and a literature review of recent developments in fabrication of hybrid materials made of stimuli responsive polymers and QDs via different approaches, including layer-by-layer assembly, macromolecular grafting and in situ $Q D$ synthesis within polymeric matrices. A particular attention is paid to the assemblies of $Q D s$ with temperature-responsive poly( $N$-isopropylacryl amide), PNIPAM.

\footnotetext{
"Parts of this chapter have been published in: Tomczak, N., Jańczewski, D., Tagit, O., Han, M-Y., Vancso, G.J. Surface Engineering of Quantum Dots with Designer Ligands Surface Design; Applications in Bioscience and Nanotechnology, Book chapter 4.3; Wiley-VCH 341 - 361 (2009)
} 



\subsection{General introduction to quantum dots}

One of the fundamental aims of life sciences is to understand the (bio)-molecules' complex spatial and temporal organization and their inter/intra-molecular interactions, from the perspective of a single molecule up to the integrative level. In order to study these interactions, researchers commonly employ fluorescent labeling for both in vivo imaging and in vitro detection [1]. The efficiency of a particular imaging or detection method depends to a large extent on the physicochemical and photophysical properties of the label used [2]. A suitable fluorescent label should fulfill certain requirements depending on the applications. For instance, an ideal fluorescent label (i) should be excitable and detectable with conventional instrumentation, (ii) should have high fluorescence quantum yield and should be stable under relevant conditions, (iv) should be soluble in relevant buffers, (v) should be suitable for surface modification for site-specific labeling [3]. Among the fluorescent labels, organic dyes and genetically encoded proteins have been most commonly used. These types of labels have known limitations. Figure 2.1 shows the absorption and emission spectra of Rhodamine 110, a commonly used organic dye. The broad absorption and emission bands mirror each other with a poor separation distance between their maxima that results in cross talk between individual dye molecules [3]. Additionally such chromophores display weak photostability, which limits their application in long-term and multiplexed imaging without complex instrumentation [4]. The problems associated with conventional organic fluorophores motivated development of alternative luminescent labels to replace the common dyes used in fluorescence detection. In this context, inorganic fluorescent semiconductor nanocrystals (quantum dots, QDs) can potentially solve many problems associated with organic fluorescent labels.

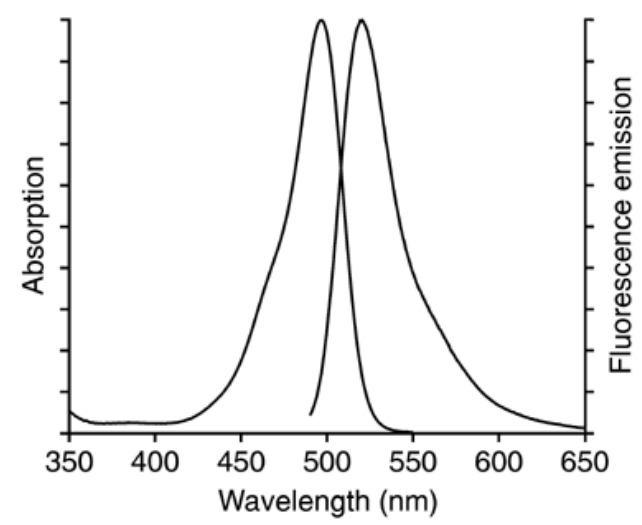

Figure 2.1. Absorption and emission spectra of Rhodamine 110. This image was taken from http://probes.invitrogen.com/media/spectra/6479ph7.gif 
QDs are semiconductor nanocrystals, composed of elements of II-VI, III-V, or IV-VI periodic groups, ranging from 2 to10 nanometers in diameter. Because of their small size, they display unique optical and electronic properties. Their stable, size-tunable, and bright luminescence, high absorption coefficients and narrow emission lines give QDs significant advantages over common organic dyes as fluorescent labels [5]. In the last decade, they have attracted tremendous attention as a new class of fluorophores with a wide range of applications in diagnostics and sensors [6]. Labeling of peptides [7], proteins [8], and DNA [9] with QDs has been achieved in addition to successful sensing applications developed for small molecules and more complex structures [10]. QDs offer possibilities such as multiplexed imaging and long term investigations due to their tunable emission wavelengths and high photostabilities.

\subsection{Optical properties of quantum dots}

Semiconductor materials are composed of a large number of covalently bound atoms. The combination of overlapping atomic orbitals leads to molecular orbitals that are closely spaced in energy, forming a virtually continuous band [11]. The electronic band structure of a material describes the 'allowed' and 'forbidden' energy levels of an electron in the semiconductor. The range of forbidden energy levels where no electron may be present is called a 'bandgap'. The highest occupied energy band is called the 'valence band'; the lowest empty band is called the 'conduction band' (Figure 2.2). For semiconductors and insulators, the bandgap refers to the energy difference between the valence band and the conduction band. The electrons in the valence band can be promoted to the conduction band upon absorption of light and leave behind unoccupied states, 'holes', in the valence band. A bound electron-hole pair is called an 'exciton'. The average physical separation between the electron and hole in an exciton is called the Bohr Radius [12].

As the size of a semiconductor approaches the size of the material's Bohr Radius, a three dimensional confinement of the electrons and holes in the nanocrystal arises. As a result of this confinement, the electron energy levels become discreet and the bandgap increases as the confinement increases [11] (Figure 2.2). 


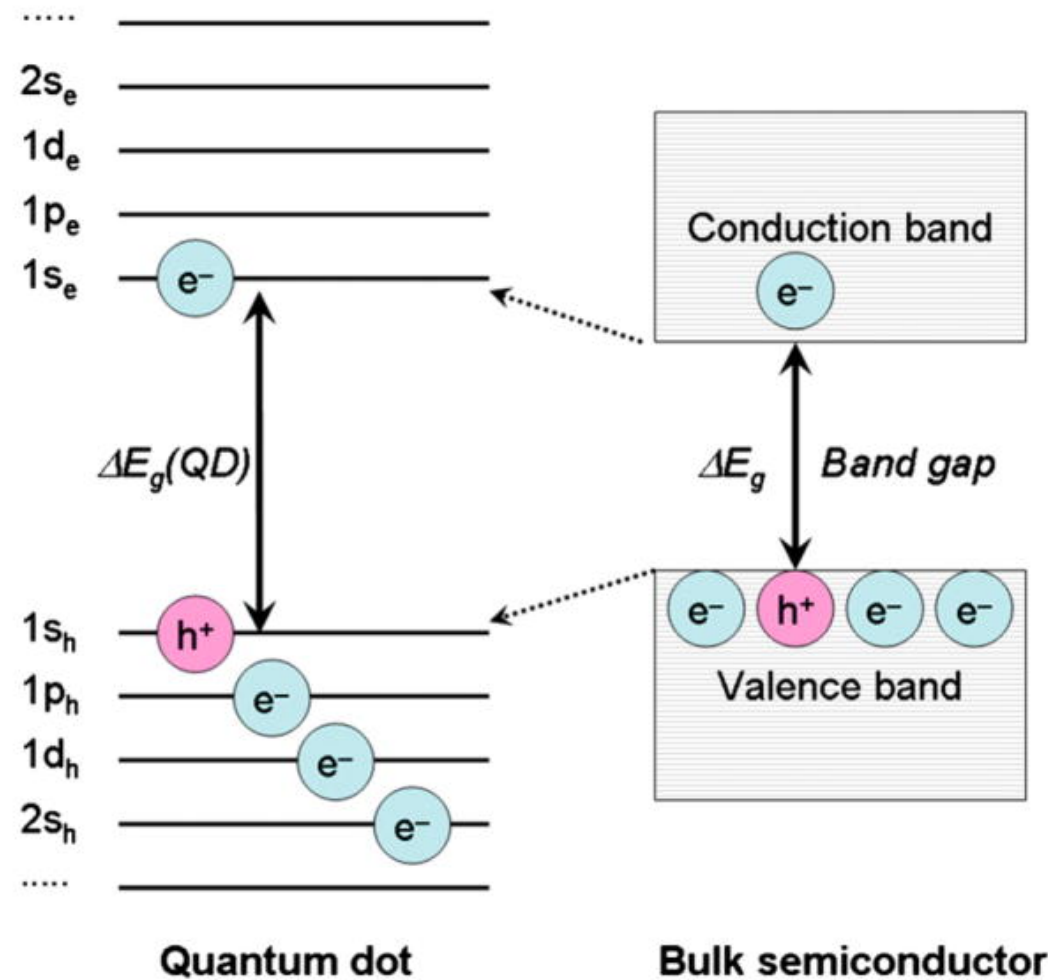

Figure 2.2. Schematic illustration of energy levels of a QD compared to a bulk semiconductor material. Quantum confinement in QDs results in discreet energy levels. This image was taken from:

http://www.ncbi.nlm.nih.gov/pmc/articles/mid/NIHMS116385/figure/F2/.

When excited with energy larger than the bandgap, an electron in the valence band can be promoted to the conduction band. As the electron falls back down across the bandgap, electromagnetic radiation with an energy corresponding to the energy it looses in the transition is emitted. Because the bandgap is size-dependent, by controlling the size of the nanocrystals one can tune the emission wavelength of the QDs. Tailored band gaps enable QDs to luminesce at wavelengths ranging from $350 \mathrm{~nm}$ to $2500 \mathrm{~nm}$ [12]. Therefore, desired photoluminescent properties can be obtained by a good control over the size of QDs (Figure 2.3.). For instance, using a single synthetic route, QDs with photoluminescence varying from green to red can be obtained [13]. 


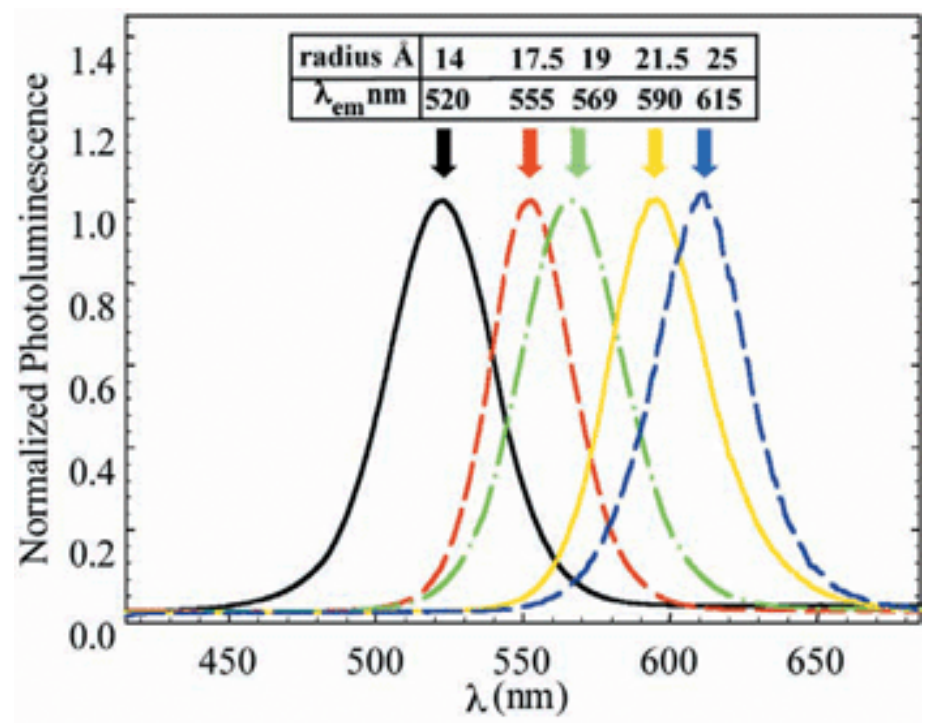

Figure 2.3. Size dependent emission of $\mathrm{CdSe} / \mathrm{ZnS}$ QDs. The excitation is at $350 \mathrm{~nm}$ in all cases. This image was taken from

http://www.nrl.navy.mil/Review02/images/materialFig9.gif.

In order to enhance the luminescence properties of QDs, an additional inorganic shell is commonly grown on the QD surface. This shell is composed of a second semiconducting material with a higher bandgap energy (Figure 2.4). The larger bandgap prevents surface oxidation, confines the excitons to the core, and passivates surface defects [14].

Probably the most commonly studied QDs are those form the II-VI group of elements [15] including CdS, CdSe, ZnS, or, CdS/ZnS, CdSe/ZnS core-shell structures.

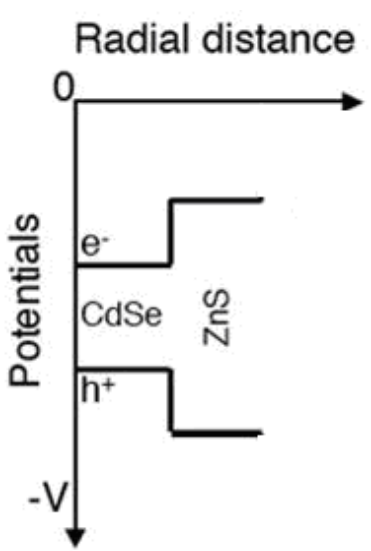

Figure 2.4. Schematic presentation of the structure and energy levels of a core/shell $\mathrm{CdSe} / \mathrm{ZnS} \mathrm{QD}$. The shell material has higher bandgap energy than the core material. All the charge carriers are confined to the core. The image was adapted from http://nanocluster.mit.edu/research.php. 


\subsubsection{Surface modification strategies for quantum dots}

QDs are usually synthesized via wet chemical methods, in which the QDs are obtained as colloidal suspensions dispersed in nonpolar solvents. Trioctylphosphine oxide (TOPO) is commonly used as the stabilizing ligand (Figure 2.5). Due to the hydrophobic nature of TOPO, QDs can not be dispersed in aqueous buffers. This is an important limitation for the use of QDs in biological applications. To disperse the QDs in biologically relevant environment, i.e., water or serum, one has to coat the QDs with appropriate capping ligands, which would prevent aggregation of the QDs [16]. Various coating chemistries for QDs have been developed including silanization [17, 18], coating with mercaptoalkanoate ligands [5], organic dendrons [19], amphiphilic polymers [20], phospholipids micelles [21], recombinant proteins [22], or oligomeric phosphines.

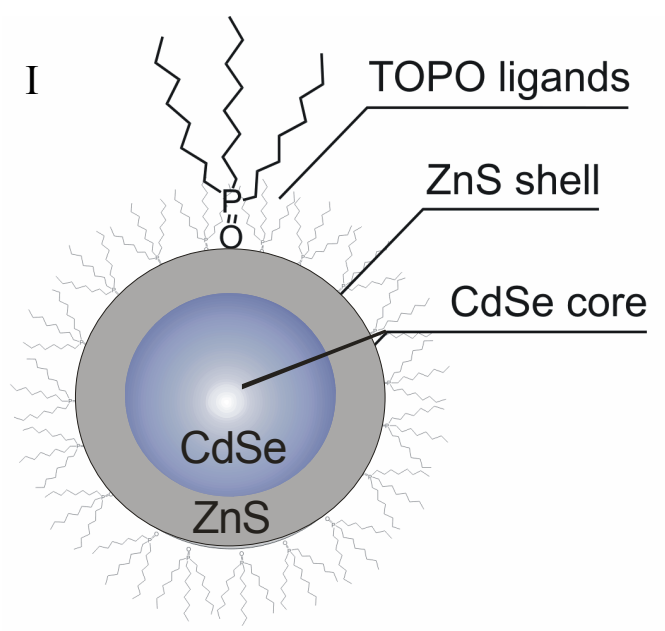

II

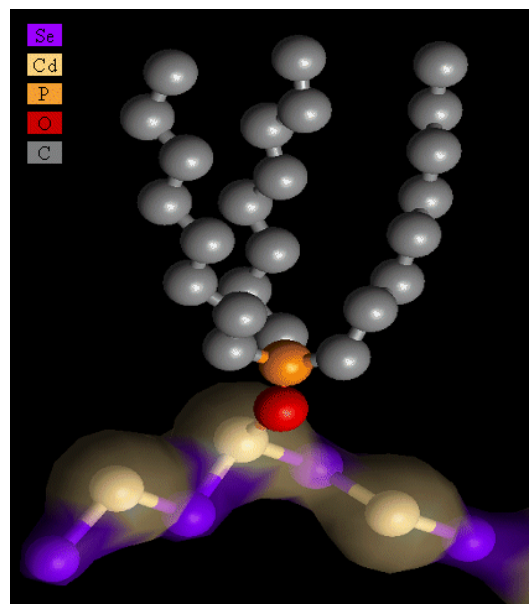

Figure 2.5. I) Schematic presentation of TOPO coated CdSe/ZnS quantum dots. II) Structure of the TOPO ligand on a CdSe surface.

Image II was taken from http://www.chemistry.manchester.ac.uk/groups/pob/exafscdse.gif

The most successful methods to render the QDs water soluble include replacement of TOPO with bi-functional ligands, such as cysteines [23], mercapto acids [24], oligomeric phosphines [25], having hydrophilic groups (carboxyl, amine, alcohol) in their structure. The second approach involves coating the TOPO layer with amphiphilic molecules. The hydrophilic part of the amphiphile is exposed to the surrounding medium and ensures water dispersability, while the hydrophobic part interacts with TOPO. For example, Dubertret et al. [26] and Geissbuehler et al. [27] reported successful encapsulation of QDs within 
phospholipid micelles using this approach. In other studies the QDs were transferred to water by covering the TOPO layer with a shell of amphiphilic polymers [28-31].

In general, polymers are good materials for encapsulation of QDs [32] (Figure 2.6). They are transparent in a wide spectral range, including the near-IR and visible region. They can be easily processed and owing to their low curing temperatures they ensure that the optical properties of the QDs are minimally affected during the processing [33].
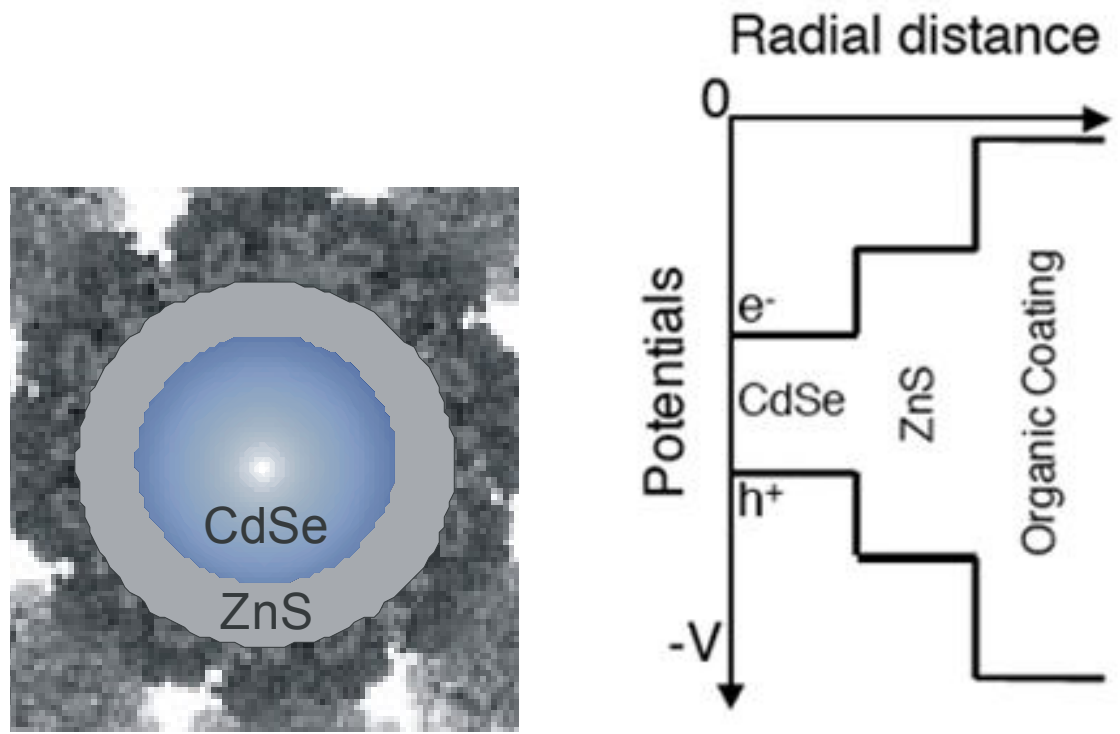

Figure 2.6. Schematic presentation of a polymer-coated $\mathrm{CdSe} / \mathrm{ZnS}$ core/shell QD. The organic coating further passivates the QD surface and offers a suitable platform for further surface modifications. The image was adapted from http://nanocluster.mit.edu/research.php.

Such organic/inorganic hybrid nanoparticles composed of polymers and inorganic QDs offer the possibility to further modify the optical and electronic properties of nanoparticles at nanoscale [34]. The organic polymer shell also determines the chemical properties of the QDs and their interaction with the environment, while the photophysical properties of the QDs are mainly governed by the size of the inorganic core [35].

\subsubsection{Poly $(N$-isopropylacryl amide) as encaging polymer for quantum dots}

Stimulus-responsive polymers, which exhibit large, rapid and reversible changes in conformation, surface characteristics or solubility in response to relatively small environmental stimuli, are often referred to as 'smart'. These polymers have attracted a great deal of attention for their 'smart' applications in combination with QDs. In a general concept the 'smart' polymers would modulate the photophysical properties of the QDs in response to 
an environmental stimulus. The stimuli may include temperature, $\mathrm{pH}$, light, electric field, ionic strength, and presence of chemicals. One of the best studied 'smart' polymers is poly(N-isopropylacrylamide) (PNIPAM). PNIPAM displays reversible conformational transition in response to changes in temperature [36] (Figure 2.7. II). This transition is associated with the lower critical solution temperature (LCST) of the polymer occurring in water at $\sim 32^{\circ} \mathrm{C}$ [37]. At temperatures below the LCST, the polymer is hydrophilic and is soluble in water. As the temperature rises above the LCST, hydrogen bonds with the water molecules are destroyed and the intra-chain hydrophobic interactions dominate. The polymer becomes hydrophobic and collapses [38-40].

I

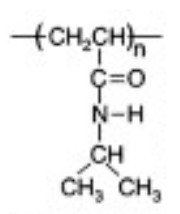

II

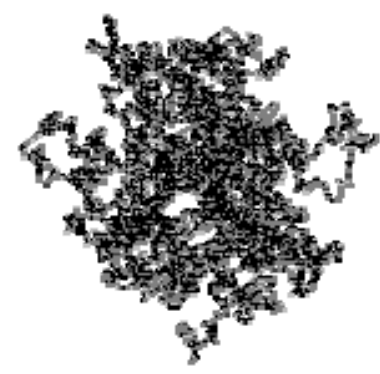

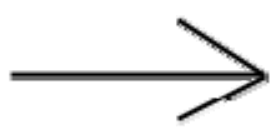

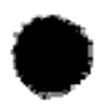

Figure 2.7. I) Chemical structure of PNIPAM. II) Schematic presentation of the PNIPAM chain collapse at LCST.

PNIPAM can be obtained in various forms including micelle [39], hydrogel [40] and tablet [41]. Moreover, PNIPAM-derived materials have been developed such as PNIPAMpolystyrene particles [42], PNIPAM-poly(butylmethacrylate) micelles [43], or PNIPAMpoly(D,L-lactide) micelles [39], where the LCST of PNIPAM can be finely tuned by adjusting the co-monomer ratios [44]. In this manner, the LCST of PNIPAM-derived materials can be increased up to around $37^{\circ} \mathrm{C}$, which is equal to the human body temperature. It has been previously shown that above LCST, PNIPAM chains interact with biocomponents, such as cells and proteins, whereas hydrated flexible chains do not interact with them [43]. Therefore, PNIPAM offers the possibility to develop new concepts in bio-related applications of QDs. For example Li et al. [45] reported the synthesis of a highly photoluminescent CdTe/PNIPAM hydrogel, and its photoluminescence was found to be sensitive to external temperature stimulus in a reversible way. The same group also prepared CdTe/p(NIPAM-acrylate) (AAc) microgels and studied their self-assembly on a glass substrate (Figure 2.8) [46]. The effects of the pH-dependent swelling properties of 
p(NIPAM-AAc) microgels and of the dipole moment of the CdTe QDs on the self assembly were studied. It was concluded that a combination of the physical and chemical properties of inorganic CdTe QDs with those of the organic polymer affected the self assembly process. The dipole moment of CdTe was an important driving force for the self assembly on a large scale and the self assembly could also be tuned by the $\mathrm{pH}$ dependent swelling behavior of the co-polymeric hydrogel. At low $\mathrm{pH}$, the aggregation morphology was fractal and dendritic on a large scale. At high $\mathrm{pH}$, the microgels aggregated to form a porous film and phase separation between the polymer and QDs occurred.

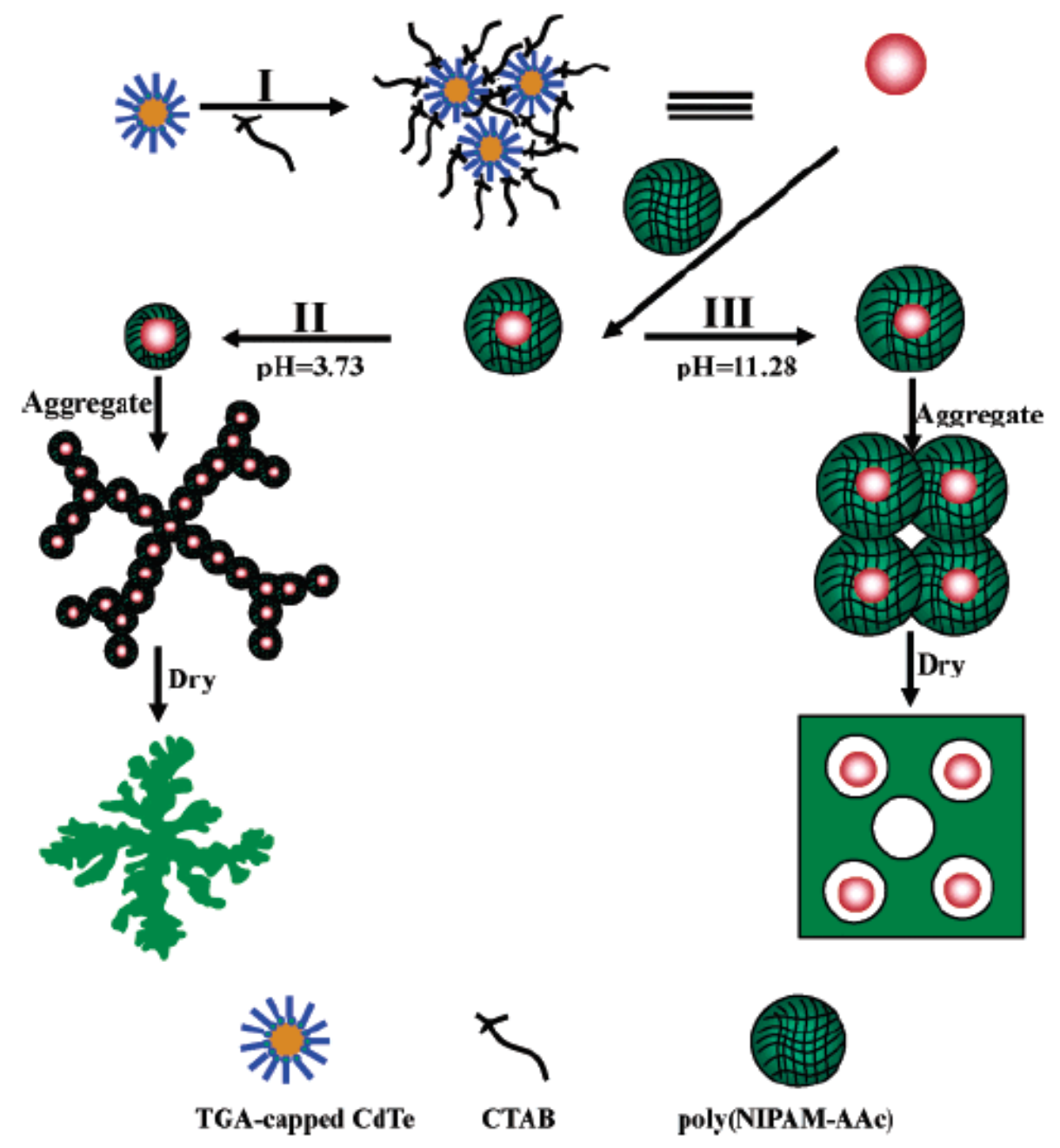

Figure 2.8. Schematic illustration of the self assembly process of CdTe/p(NIPAM-AAc) microgels. Addition of CTAB into the QD solution results in formation of QD complexes (I). These complexes are mixed with p(NIPAM-AAc) microgels. At low $\mathrm{pH}$ the microgels are in a shrunken state (II) and their self assembly upon drying is investigated. The self assembly process is also investigated at high $\mathrm{pH}$ values (III), where the $\mathrm{p}$ (NIPAM-AAc) microgels are in a swollen state. This image was taken from [46]. 
Wang et al. [47] have employed NIPAM and 4-viniylpyridine (VP), a pH responsive monomer, to fabricate pNIPVP spheres as colloidal carriers and to confine CdTe nanocrystals of different sizes within these hydrogel networks by changing the external $\mathrm{pH}$. They also studied the controlled release of QDs by $\mathrm{pH}$ stimulus (Figure 2.9.). By absorbing the QDs of different size, they were able to achieve multicolour-coded microspheres with a LCST around $34^{\circ} \mathrm{C}$. This system was designed to be used as a delivery agent for the QDs and their bioconjugates within the human body.

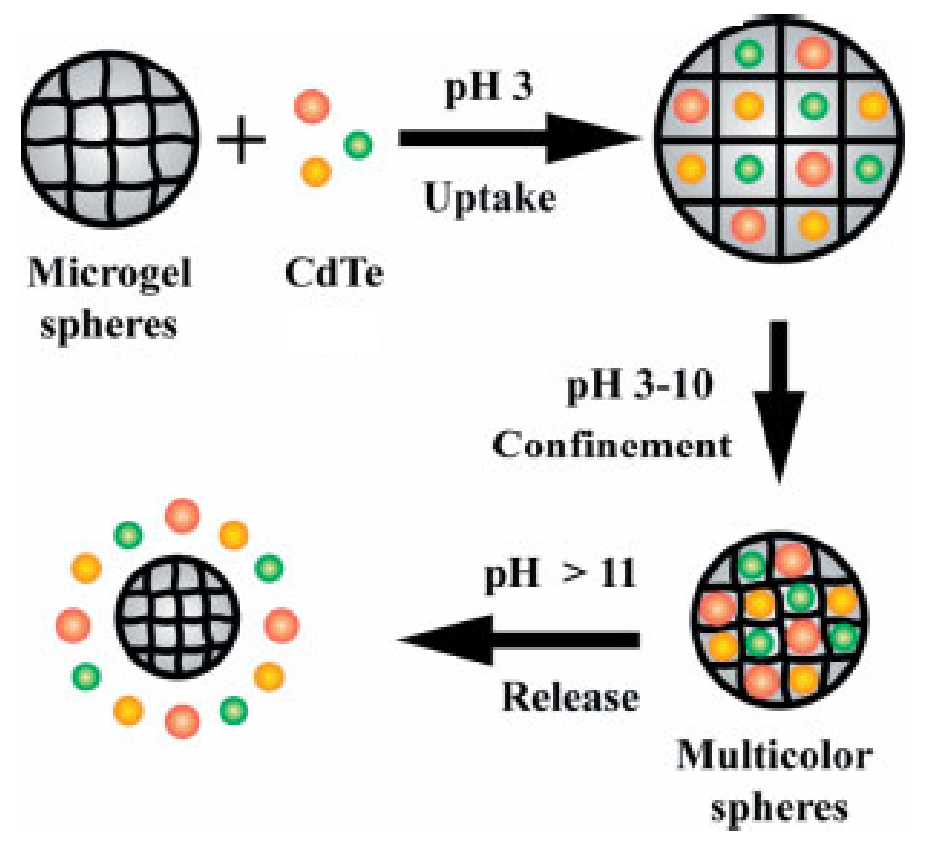

Figure 2.9. Schematic illustration of the loading of CdTe QDs in pNIPVP hydrogels and their controlled release by $\mathrm{pH}$ stimulus. The $\mathrm{pH}$-dependent phase transition of pNIPVP hydrogels is used for the internalization of QDs at $\mathrm{pH}$ 3. Upon increasing the $\mathrm{pH}$, the hydrogel shrinks and the QDs are confined within the hydrogel. The release of QDs is achieved by increasing the $\mathrm{pH}$ to above 11. This image was taken from [47].

When designing QD/polymer hybrid materials, a number of factors should be considered such as the size and shape of the polymer matrix, the amount of QDs to be entrapped within the matrix, as well as the spatial distribution/localization, separation and orientation of the QDs within the matrix. The fluorescent hybrid nanoparticles should be monodisperse and have relatively small size and large saturation intensity and be highly luminescent [48]. The requirements for their applications in single-molecule biological 
studies are even more stringent: they must be biocompatible, non-cytotoxic, chemically stable and offer conjugation chemistries for attachment of recognition molecules to their surfaces.

The size of the host matrix may be few hundreds to tens of nanometers depending on the fabrication procedure and desired applications. QDs with thicker polymer coating tend to have better photostabilities and higher QYs, whereas QDs with thinner coatings would be more suitable as intracellular probes [48]. The size of the matrix plays also an important role in limiting the allowed number of QDs per matrix volume so that e.g. fluorescent resonance energy transfer (FRET) is prevented. Incorporation of QDs into spherical nano sized matrices is of interest for both fundamental studies on light-matter interactions and for practical applications [49]. These dot-in-a-dot structures confine electrons and photons in all three dimensions. The real success in developing polymer/QD nanohybrid materials is achievable only when the above requirements are satisfied. This necessitates a control over the size and shape of the matrix, and over the amount, spatial distribution/localization, separation and orientation of the QDs within the matrix.

\subsection{Designing polymer/quantum dot hybrid assemblies}

Prior to realizing most applications, QDs must be functionally integrated into matrices [34]. Some of the main routes, via which polymer/QD nanohybrid particles have been prepared, include layer-by-layer deposition of QDs on oppositely charged beads by electrostatic interactions, loading QDs into polymer beads during the synthesis of the polymeric nanoparticles, grafting polymeric shells from or to the QD surfaces, and in situ synthesis of QDs within polymeric matrices.

\subsubsection{Layer-by-layer electrostatic assembly approach}

Among the assembly techniques used to build functional structures, the electrostatic layer-by-layer (LbL) assembly evolved as a powerful method for the construction of supramolecular hybrid architectures by sequential absorption of oppositely charged polyelectrolytes [50], which enabled formation of functional thin film architectures [51]. By this simple and versatile method, it is possible to exert molecular-level control over the architecture, composition, and thickness of the films $[52,53]$. The precise control that is offered by LbL assembly leads to remarkable improvements in organic optoelectronic devices owing to the large interfacial areas for charge separation and creation of efficient charge transfer pathways [54]. 
Recently, a variety of schemes were introduced for the fabrication of QD multilayer films prepared with oppositely charged species. Mamedov et al. used positively charged polyelectrolyte (poly(diallyldimethylammonium chloride), PDDA) for the preparation of graded QD films [55]. Zhang et al. reported the assembly of aqueous CdTe nanoparticles with $\mathrm{N}$-vinyl carbazole/4-vinyl pyridine copolymer [56]. Kotov's group prepared a monolayer film of QDs (CdSe/PDDA or CdSe/CdS/PDDA) with homogenous, nearly close-packed coverage with little aggregation. [57]. However, these studies paid little attention to the photoluminescence (PL) efficiency of QDs in the films. Lesser et al. reported that the LbLassembled QDs had only 5\% PL efficiency, although the initial PL efficiency of QDs in an aqueous colloidal solution was equal to $20 \%$ [58].

For the fabrication of devices with patterned layers of QDs, one of the issues to consider is selectivity and non-specific interactions. Zhou et al. showed that the selectivity over patterned surfaces exhibited in the first few layers can decrease dramatically and almost disappears after tens of bilayers are assembled [59]. Therefore, it is important to improve the selectivity to the patterned features while reducing the non-specific interactions. The same group reported reduced non-specific interactions through the modification of QD surface coatings and employing a polymer with a hydrophilic backbone. Their method was based on patterning a gold substrate with self assembled monolayers (SAMs) of alkyl thiols terminated with hexa(ethylene glycol), which acted as the resistive coating due to its resistance against non-specific adsorption. Linear poly(ethyleneimine) (LPEI), a positively charged polymer, was used as the 'assembly partner' for 2-mercaptoethanesulfonic acid (MESA) terminated QDs (Figure 2.10). Using the LbL approach, the authors were able to selectively build up 3D fluorescent surface patterns. 

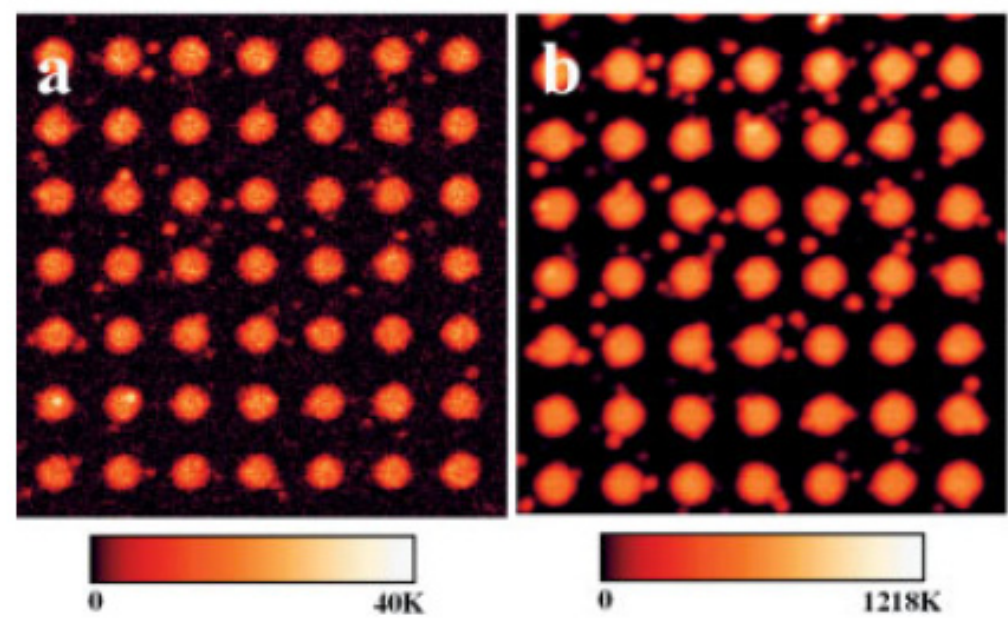

Figure 2.10. Fluorescence images at different stages of the LbL assembly of MESA-QDs and LPEI on a patterned substrate. a) After 2 cycles, b) after 19 cycles of assembly. Image size: $30 \mu \mathrm{m} \times 30 \mu \mathrm{m}$. High signal-to-noise ratio shows that very little non-specific adsorption occurs during the assembly process. This image was taken from [59].

Jaffar et al. [60] coated mercaptoacetic acid (MAA) treated QDs with cationic poly(allylamide) (PA) and subsequently with poly(vinylsulfonic acid) (PVSA) (Figure 2.11.).

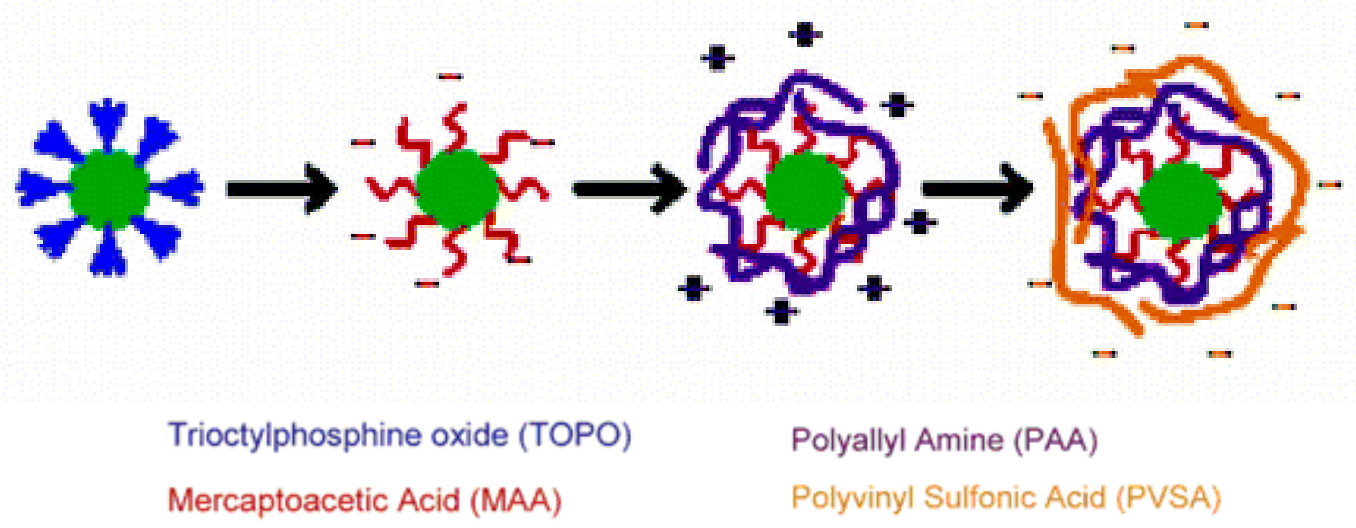

Figure 2.11. Modification scheme of $\mathrm{CdSe} / \mathrm{ZnS}$ QD by LbL. The ligand exchange reaction is performed to replace TOPO with MAA to render QDs water soluble and negatively charged. Subsquently, positively (PAA) and negatively (PVSA) charged polyelectrolytes are deposited on the QD surface. This image was taken from [60]. 
In order to produce structured arrays, PAA-coated QDs (green) and MAA-treated QDs (red) were deposited on a glass substrate patterned by hyaluronic acid (Figure 2.12).

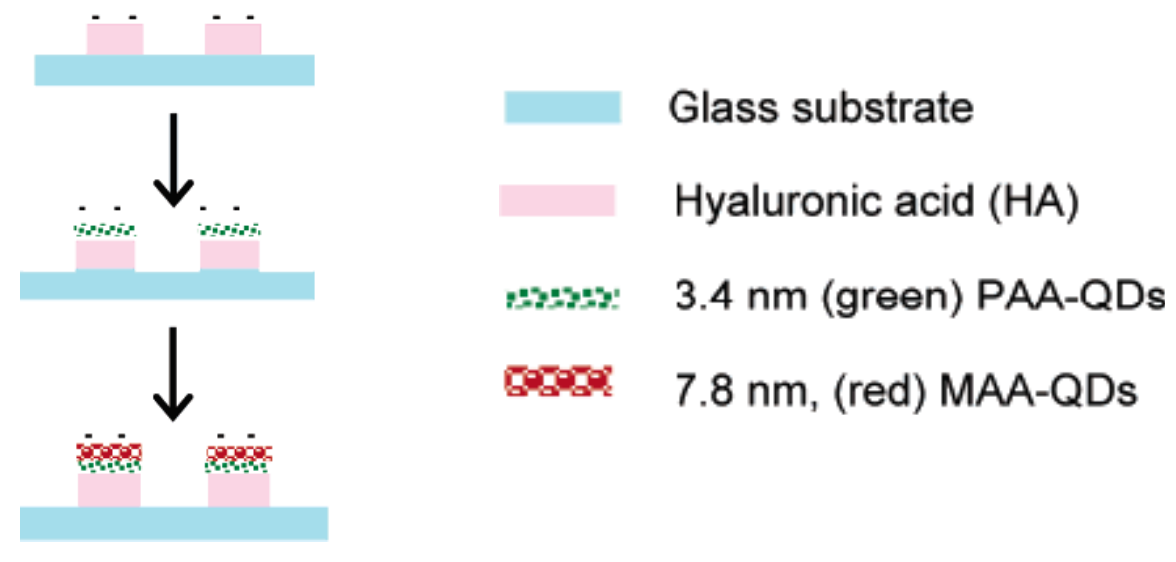

Figure 2.12. Self-assembly scheme of modified QDs on HA patterned glass substrate. Anionic MAA-QDs and cationic PAA-QDs bind to the HA patterns on the glass substrate. This image was taken from [60].

As an alternative to using electrostatic forces [45, 53, 61-63], covalent LbL assembly of polymers and QDs was also performed [52]. Liang et al. prepared robust and smooth, functional QD/polymeric thin films [52]. The QD/polymer hybrid structures displayed promising properties for applications in light-emitting diodes, photovoltaics, lasers and biosensors [64].

In summary, LbL assembly of QDs using polymers offers control of their spatial organization over a range of length scales. These nanostructures prove useful as building blocks for opto-electronic device fabrication.

\subsubsection{Loading quantum dots into polymer matrices during the polymer synthesis}

Among the methods used for the incorporation of QDs into polymeric matrices, loading of QDs through emulsion [65] and suspension [66] polymerizations allows one to disperse the QDs through the volume of a spherical polymeric particle. A general procedure involves polymerizing the monomer in the presence of dispersed QDs. However, there are some challenges related to this approach, such as control over the amount and location of the QDs within the polymeric particles and control of the colloidal stability and monodispersity of the polymeric particles [65]. 
Sheng et al. [49] reported a strategy for the incorporation of $\mathrm{CdSe} / \mathrm{ZnCdS} / \mathrm{ZnS}$ QDs into polystyrene (PS) microspheres by using functionalized oligomeric phosphine (OP) ligands. The TOP-coated QDs were first dispersed in OP/DMF solution, which was followed by prepolymerization of MMA groups to form a polymeric shell around the QDs. Formation of polystyrene particles in the presence of surface-modified QDs was achieved via free radical polymerization at high temperatures. (Figure 2.13.).

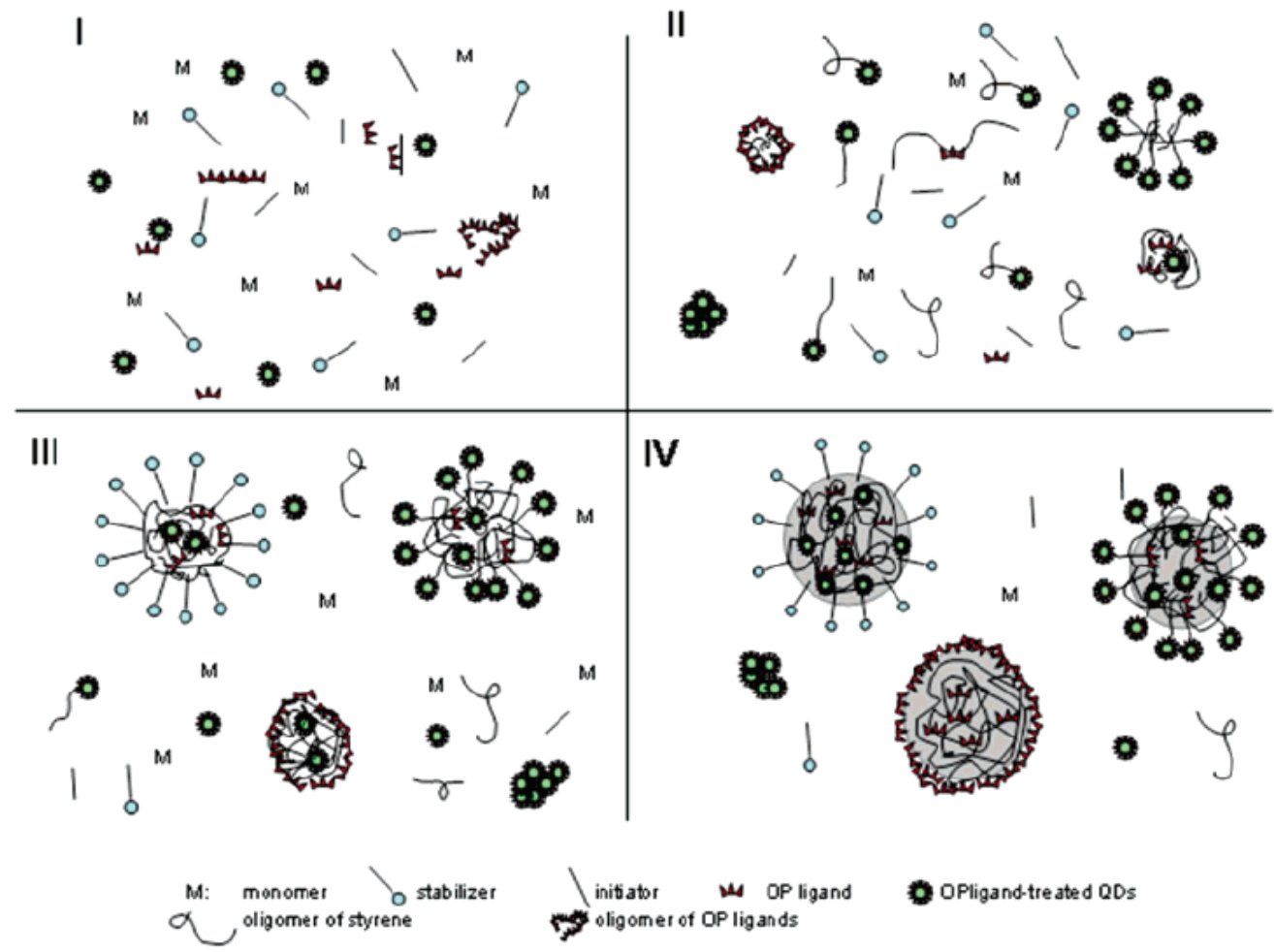

Figure 2.13. Schematic illustration of the formation of QD-PS microspheres. The starting reaction mixture contains styrene monomer, initiator, stabilizer, OP ligand, and QDs treated with the OP ligand (I). Polymerization begins as the initiator decomposes (II). As the polymer chains reach a critical length they begin to aggregate into small particles which are stabilized by the stabilizer molecules (III). The polymerization continues inside the particles until all the monomer units are used up (IV). This image was taken from [49].

Fluorescence imaging of the particles (Figure 2.14) showed that all the QD/PS hybrid particles were well separated, proving that QDs had been incorporated within the polymeric matrix and are still well protected by OP ligands. 


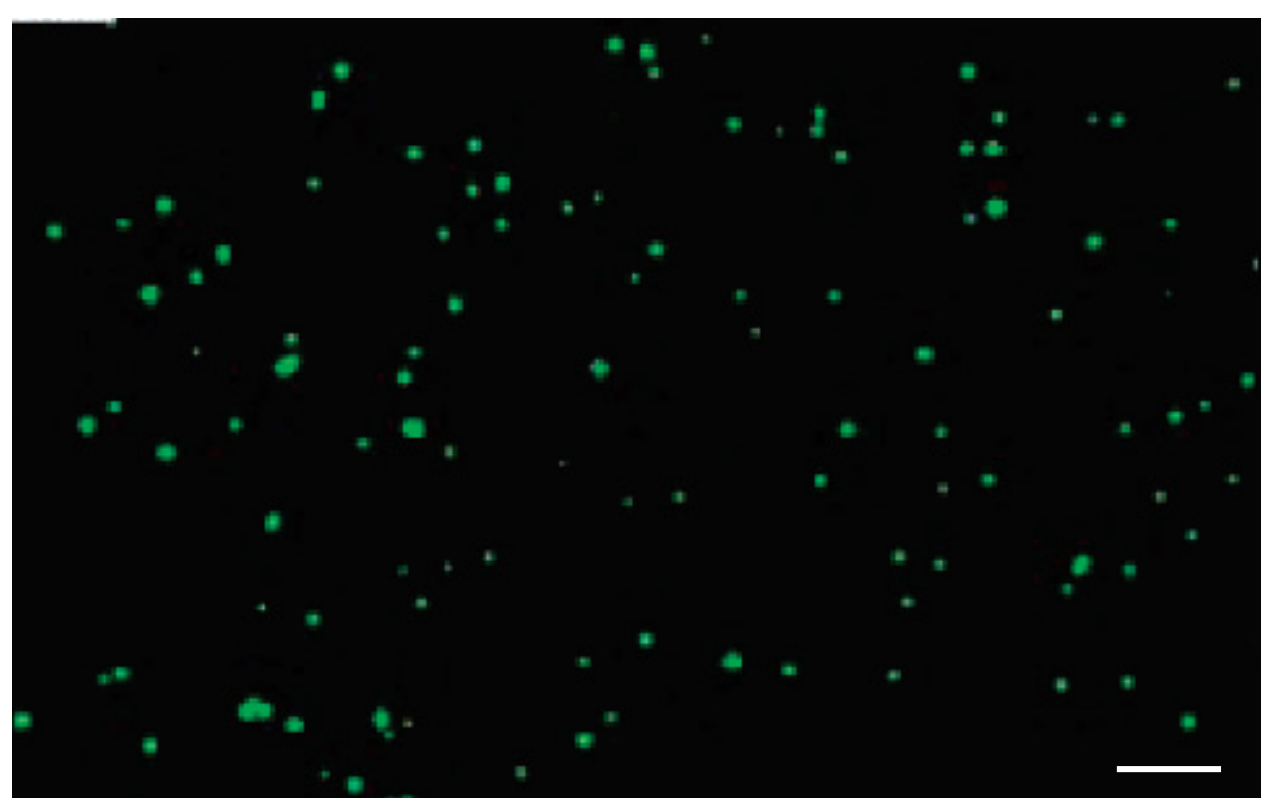

Figure 2.14. Fluorescence image of QD/PS particles. Scale bar: $5 \mu \mathrm{m}$. This image was taken from [49].

As an alternative strategy, radical polymerization in miniemulsion of $\mathrm{QD} /$ polymer nanocomposites without further modification of the QD surface was reported $[65,67,68]$. Some features of the miniemulsion polymerization technique provide potential advantages for the encapsulation of organically capped QDs, namely, the ability to nucleate all the droplets containing the inorganic nanoparticles, a good control of the droplet size and size distribution, and the direct dispersion of the QDs within the oil phase [67]. The as-prepared QDs have their surface passivated with TOPO molecules, leaving the hydrophobic octyl chains directed outward $[69,70]$. As a result, TOPO capped QDs are easily dispersed in a nonpolar media such as several organic solvents or in viscous liquid monomers. Therefore, in order to encapsulate the QDs within polymers via the miniemulsion polymerization strategy, there is no need for further surface derivatization.

In the study reported by Esteves et al. [67], the incorporation of QDs into the polymer particles was achieved via polymerization of a mixture of TOPO-coated CdS or CdSe QDs and the monomer (Figure 2.15.). Two polymeric matrices, polystyrene (PS) and poly(n-butyl acrylate) (PBA), were investigated. In both cases, homogenous nanocomposites were obtained. 


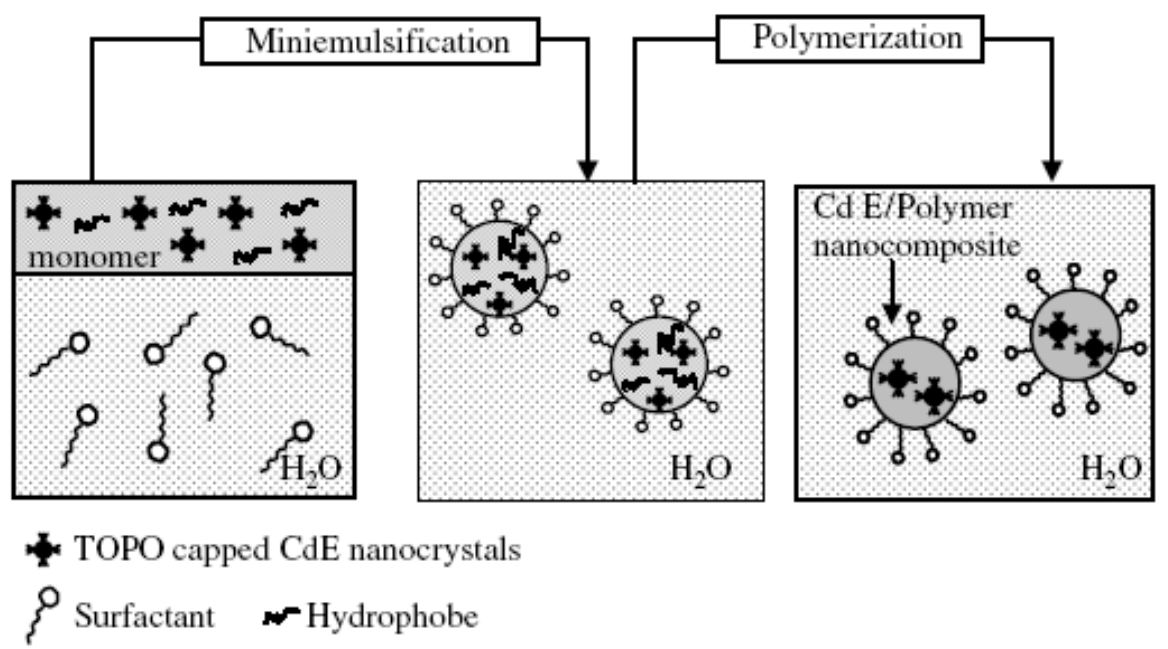

Figure 2.15. Schematic representation of the miniemulsion polymerization of QD/polymer nanocomposites. TOPO-coated QDs are dispersed in the monomer and sonication of the monomer/water mixture results in miniemulsion formation. The polymerization of the monomer droplets results in QD/polymer nanocomposites. This image was taken from [67].

Due to its high-luminescent properties, the PL of the CdSe/PBA nanocomposite was analyzed in more detail. The emission spectra of the CdSe/PBA nanocomposite and the respective CdSe QDs are shown in Figure 2.16. The narrow PL emission band is blueshifted from the bulk PL due to a strong quantum confinement effect. Therefore the emission observed in the CdSe/PBA nanocomposite proceeds directly from the unique properties of the

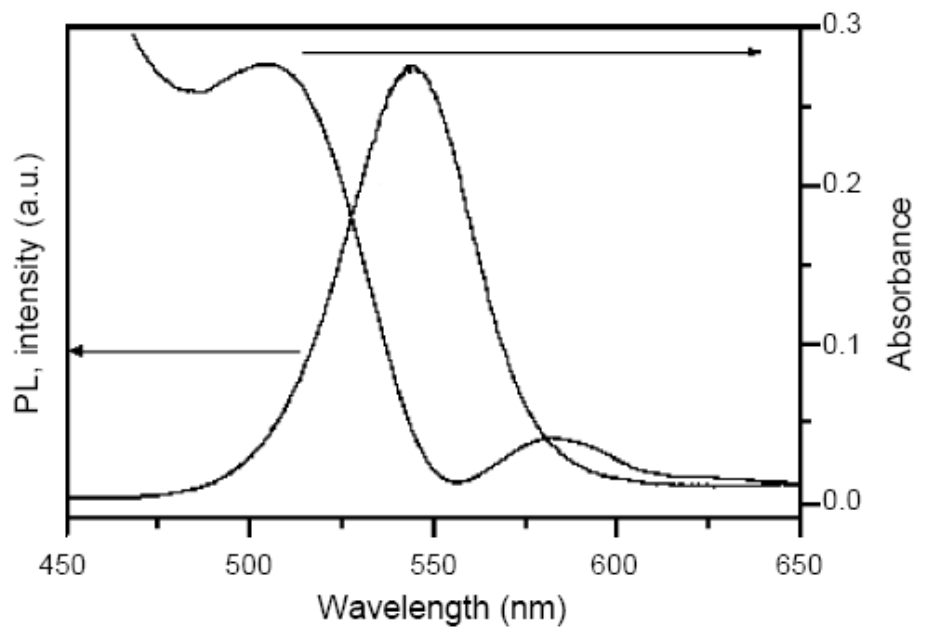

Figure 2.16. PL spectra of $\mathrm{CdSe}$ and $\mathrm{CdSe} / \mathrm{PBA}$ nanohybrid particles at room temperature. This image was taken from [67]. 
constituent QDs. In this experiment, contrary to some other reports where conventional free radical initiators (such as AIBN) degraded the dots and totally quenched their optical properties [71], QD degradation or PL quenching was not observed. This is probably due to dispersion of QDs within monomer droplets and use of a water soluble initiator, KPS, which might reduce the contact between QDs and the free radicals.

In order to overcome the quenching problems associated with the use of free radicals during the polymerization Zhang et al. [72] used an alternative method in which aqueous nanocrystals were used instead of hydrophobic QDs. The maximum PL was reported to be retained when the thickness of the Cd-thiol complexes around QDs was increased under proper conditions. This was also proven by some other reports where a shell of Cd-mercapto carboxylic acid complexes was formed around CdTe QDs [73]. This structure improved both the PL intensity and stability of aqueous QDs [74].

Considering all the results mentioned above, miniemulsion polymerization seems to be a promising approach for the encapsulation of hydrophobic QDs within polymeric matrices. However, fabricating monodisperse hybrid particles with homogenously distributed QDs still remains a challenge.

\subsubsection{Grafting approaches}

Growing polymer chains around inorganic cores is one of the most popular methods used to obtain core/shell structures. In this approach, polymer chains are tethered by one end to the surface of the core particle [75]. Generally there are two methods to chemically attach polymer chains to a surface:

i) Grafting-to method;

ii) Grafting-from method.

In grafting-to method, the end-functionalized polymers react with an appropriately functionalized surface, i.e., this method involves irreversible grafting of a presynthesized polymer chain $[75,76]$. This method has certain limitations including:

- Relatively low surface coverage;

- Restricted diffusion of the polymer chain-ends to the surface;

- Island formation due to steric crowding of the reactive sites by the already-grafted polymers; 
- Lack of complete control over the growth of stable polymer brushes at nanoscale [77].

The second approach involves initiation of polymerization from the surface of the particles, which are functionalized by initiators [75]. This is called the grafting-from method and it provides a greater control over the density of the grafts [78].

Both methods mentioned above are used mainly in combination with free radical polymerizations [78]. Greater control over the polymerization process is possible by using living polymerization or employing controlled radical polymerization scheme, where the concentration of the radicals is kept at minimum by equilibrating the reactive radicals with their reversibly-terminated counterparts. In such systems, surface initiation can be combined with atom transfer radical polymerization (ATRP), nitroxide-mediated radical polymerization (NMRP) or photoiniferter-controlled polymerization, where the reaction time determines the thickness of the polymeric shell [79].

Using the grafting-from approach, a number of research groups synthesized polymer brushes on silica [80, 81], gold [82], QDs [71, 83], and magnetic nanoparticles [84, 85].

Regarding QDs, Farmer and Patten prepared $\mathrm{CdS} / \mathrm{SiO}_{2}$ core/shell nanoparticles and modified their surfaces with an ATRP initiator (Figure 2.17) [83]. The initiator-modified nanoparticles were then used in the polymerization of methyl methacrylate (MMA).

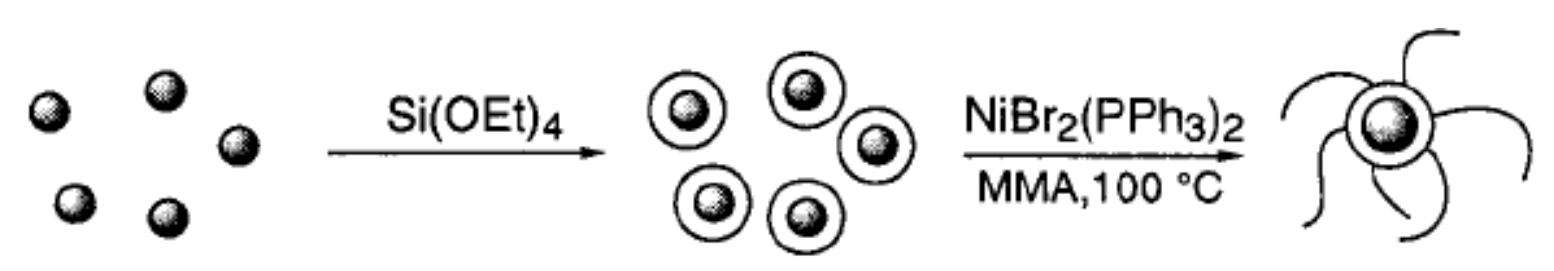

Figure 2.17. Schematic representation of preparation of $\mathrm{Cd} / \mathrm{SiO}_{2} / \mathrm{PMMA}$ nanoparticles via reverse microemulsion. Addition of $\mathrm{Si}(\mathrm{OEt})_{4}$ results in formation of $\mathrm{SiO}_{2}$ coating on the QD surface. After modification of the QD surface with the initiator, the polymerization proceeds to form $\mathrm{QD} /$ polymer hybrids. This image was taken from [83].

The TEM images of the particles (Figure 2.18) demonstrate that, it is possible to obtain QD/polymer hybrid particles with a single QD located in the centre of the matrix. This is difficult to achieve using other methods to incorporate QDs in polymeric matrices. 


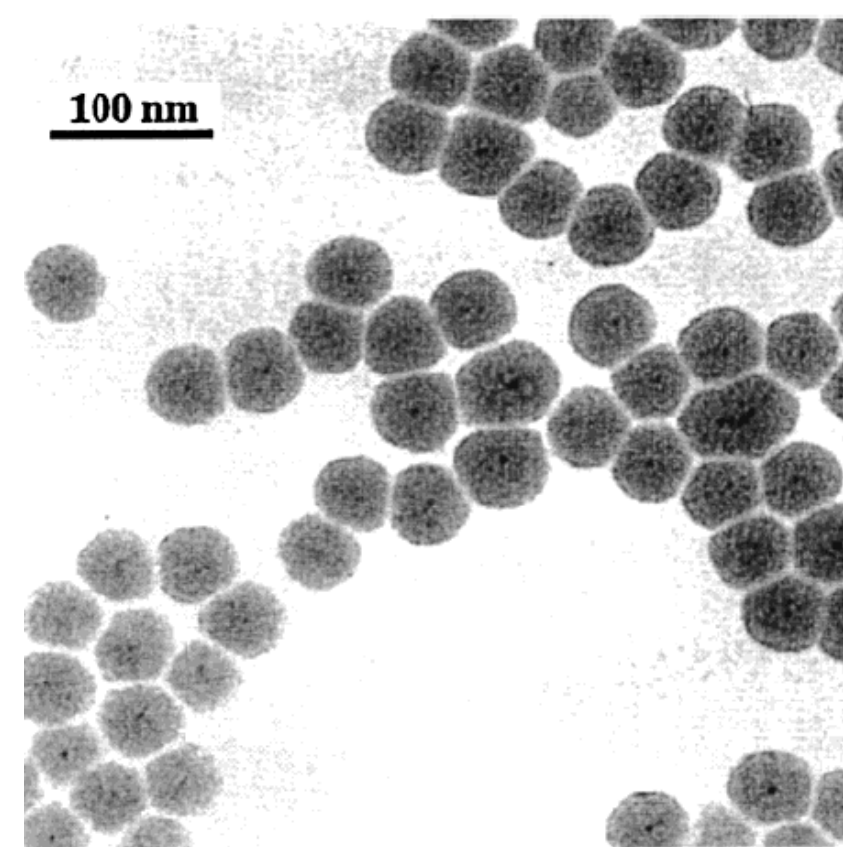

Figure 2.18. TEM image of $\mathrm{QD} / \mathrm{SiO}_{2}$ particles. This image was taken from [83].

In another study Sill and Emrick [71] reported polystyrene and poly(styrene-methyl methacrylate) copolymer brushes grown directly from the surface of CdSe nanoparticles by nitroxide-mediated controlled free radical polymerization. Since free radicals can quench the fluorescence of the CdSe nanoparticles, nitroxide-mediated polymerization allows for the preparation of polymer-nanoparticle composites while maintaining the fluorescence of the nanoparticles.

In summary, grafting polymers on the QD surfaces is a promising approach for the preparation of hybrid nanoparticles. However, more attention should be paid to the control over the polymerization reactions as well as over the grafting density of the polymer chains. In addition, new protocols should be developed in order to minimize the influence of the reaction conditions on the PL intensity of the QDs.

\subsubsection{In situ synthesis of quantum dots within polymeric matrices}

Synthesis of semiconductor nanoparticles in geometrically restricted environments has been well-studied [86]. The studies included block copolymer micelles [87], reverse micelles and micro emulsions [88], organic-inorganic matrices [18] and hydrogels [89, 90] as nanoreactors for the synthesis of semiconductor nanoparticles. 
The preparation of CdS in block ionomer reverse micelles in an organic solvent was reported by Moffitt et al. [91]. CdS was precipitated within the ionic cores of a PS-b-PACd diblock ionomer. In a follow-up study, aqueous solutions of polystyrene-b-poly (acrylic acid) (PS-b-PAA) compound micelles containing QDs were obtained [92].

Other preparations of CdS QDs involved also triblock copolymers such as hydroxylated poly-(styrene-b-butadiene-b-styrene) micelles in toluene [93] and poly(ethylene oxide)-b-polystyrene-b-poly (acrylic acid) triblock copolymers (PEO-b-PS-b-PAA) [91].

In the study reported by Duxin et al. [92] the authors obtained different polymer morphologies from the same triblock copolymer, which consisted of a cadmium acrylate (CdAcr) core, surrounded by PS chains and a PEO corona (Figure 2.19). The use of block copolymers in QD synthesis allows also for precise localization of the QDs within the bulk matrix, or in the surfaces of inverted micelles [87, 94-96].

In another study by Chu et al. [97] a synthetic route for the preparation of luminescent and rodlike $\mathrm{CdS}$ nanocrystals embedded in poly(BA-co-GMA-co-GMA-IDA) (PBGM) copolymer templates by soap-free emulsion copolymerization was presented. In this study, GMA-IDA groups within the copolymer acted as coordination sites for chelating $\mathrm{Cd}^{2+}$, at which nanosized CdS nanocrystals were grown by the dry method $\left(\mathrm{H}_{2} \mathrm{~S}\right)$ and the wet method $\left(\mathrm{Na}_{2} \mathrm{~S}\right)$ (Figure 2.20). The hybrid semiconductor-polymer composites prepared with the above procedure are stable and free from other capping molecules.

Recently, hydrogels have also been investigated as nanoreactors for producing semiconductor or metallic nanoparticles $[89,90]$. By choosing a suitable polymer, the swollen-shrunk states of the hydrogels can be effectively controlled by external stimuli like temperature, $\mathrm{pH}$, electric field, solvent, etc.

The incorporation of QDs into the hydrogels is usually based on the "breathing in" technique, where the dry hydrogel is swollen with an aqueous solution containing the preformed nanoparticles $[89,90,97]$. An alternative approach is based on in situ formation of the nanoparticles using appropriate ionic precursors. This method has been successfully employed to form $\mathrm{CdS}$ nanoparticles on the surface of poly(methyl methacrylate-comethacrylicacid) latexes [98] or in the interior of poly(N-isopropylacrylamide-co-acrylic acid) microgels [99]. Because of the acrylate anions, these gels are negatively charged, and the introduction of the precursors was performed via ion exchange of the latex or microgel counterions with $\mathrm{Cd}^{2+}$. Furthermore, the formation of the nanoparticles was localized around the acrylate anions, which offered an opportunity to control the location of the QDs within the matrix. 


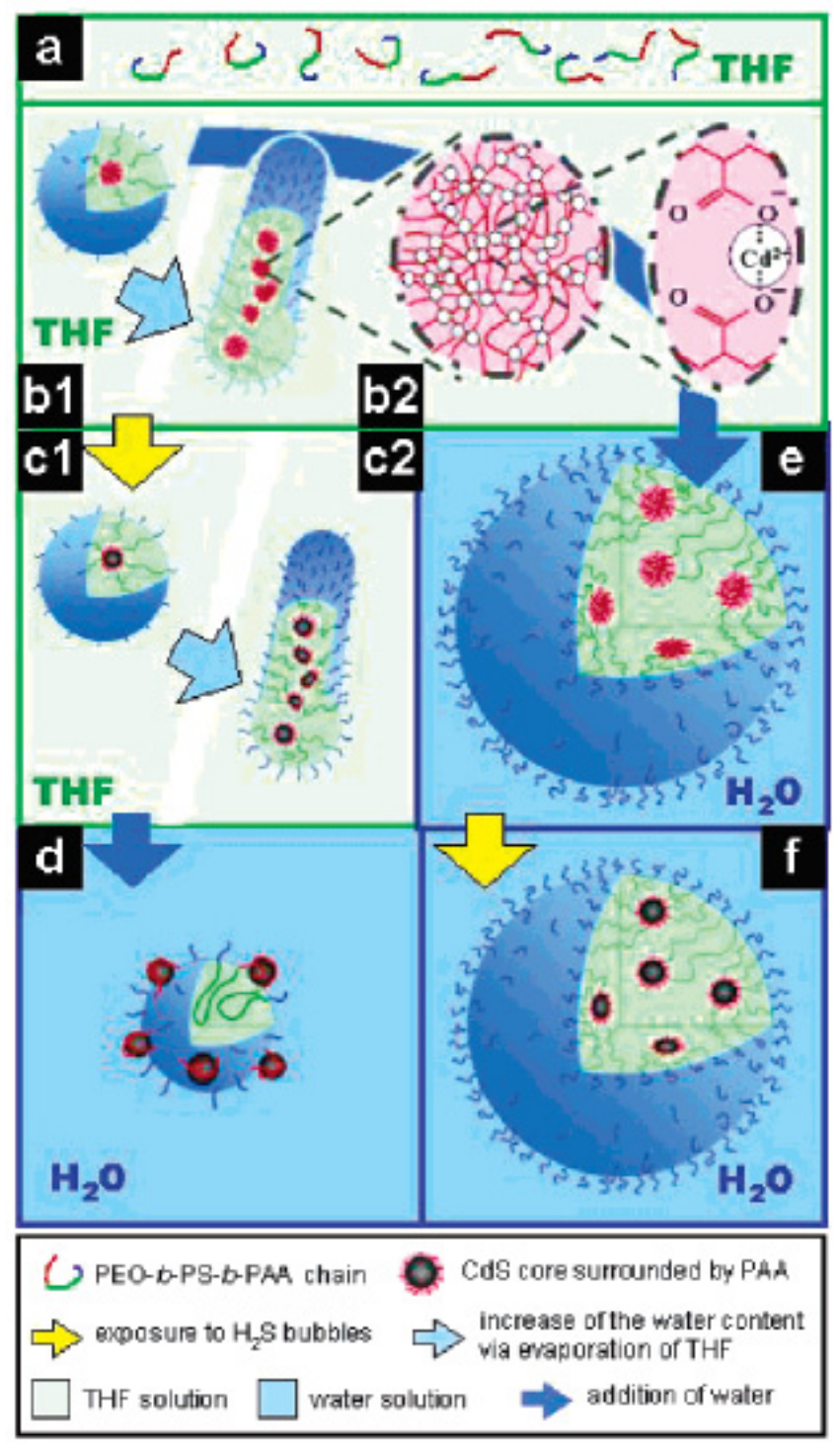

Figure 2.19. Schematic illustrations of the formation of PEO-b-PS-b-PAA assemblies. (a) Single triblock copolymer molecules in THF. (b) Ionically crosslinked triblock micelles. (b1) Primary spherical inverse micelles (PSIMs) in THF; (b2) wormlike micelles at higher water content. (c) Triblock copolymer structures with CdS quantum dots. (c1) Spheres in THF; (c2) rods in water-rich solutions. (d) PS core micelles in water, surrounded by CdS nanoparticles. (e) Multicore cadmium acrylate supermicelle (SM) structures, following the change of the solvent to water of the PSIMs shown in part b. (f) Water soluble SM triblocks with CdS cores. This image was taken from [92]. 

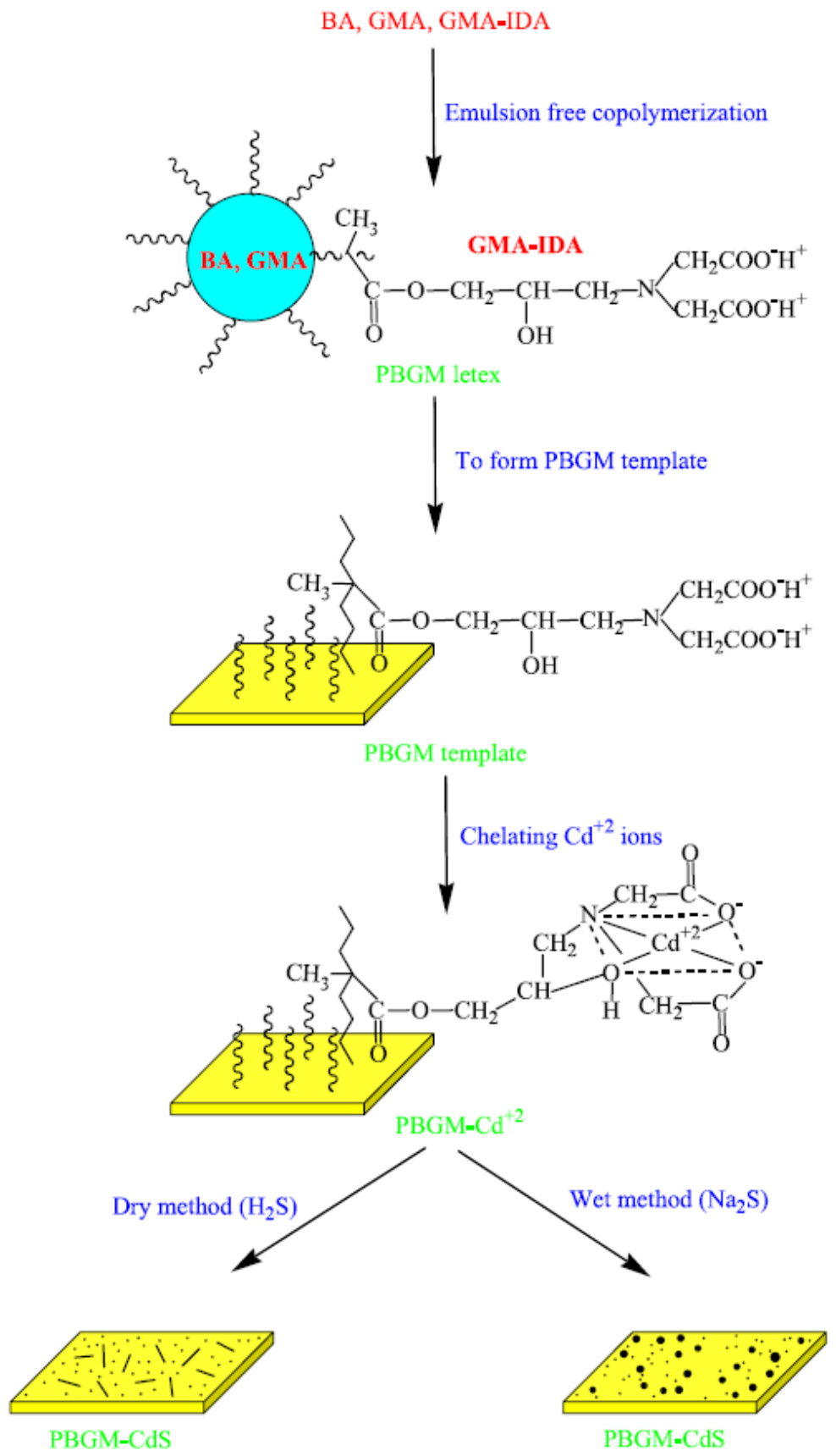

Figure 2.20. Schematic illustration of the preparation of luminescent and rodlike CdS nanocrystals embedded in poly(BA-co-GMA-co-GMA-IDA) (PBGM) copolymer templates. This image was taken from [97]. 
Bekiari et al. used the in situ synthesis to prepare CdS nanoparticles in a nonionic hydrogel, based on poly(N,N-dimethylacryl-amide), PDMAM [86]. Contrary to previous studies, the distribution of the CdS nanoparticles in the PDMAM hydrogel is expected to be homogeneous throughout the whole hydrogel volume since PDMAM is a nonionic, hydrophilic polymer. A special attention has been paid to the fate of the QDs as a function of the degree of swelling of the hydrogel.

In summary, preparation of QDs/hydrogel nanohybrid particles is an important issue for the development of luminescent materials. The materials can serve as luminescent probes of macromolecules of biological importance.

\subsection{Conclusions}

Many future materials and devices based on QDs require their incorporation and organization in polymeric matrices. The growing need for photonic materials and devices encouraged the development of many different strategies to produce polymer/QD hybrid structures. The appropriate choice of a strategy depends mainly on the final application of the hybrid materials. For instance, if the material is designed to be used in biological applications, in addition to its luminescence efficiency, the compatibility of the material with the biological systems should be also considered. The fabrication methods which have been reported to date (for a recent review see [100]) have advantages and drawbacks. This makes the development of new routes for producing QD/polymer nanohybrid materials a hot scientific topic, since simultaneous control over the size and shape of the matrix, and over the amount, spatial distribution/localization, separation and orientation of the QDs within the matrix still remains a challenge to be solved.

\subsection{References}

1- $\quad$ Miyawaki, A., Dev. Cell 2003, 4: p. 295

2- Waggoner, A., Curr. Opin. Chem. Biol. 2006, 10: p. 62

3- Resh-Genger, U.; Grabolle, M.; Cavaliere-Jaricot, S.; Nitschke, R.; Nann, T., Nature Methods 2008, 5: p. 763

4- Louis, C.; Bazzi, R.; Marquette, C. A.; Bridot, J. L.; Roux, S.; Ledoux, G.; Mercier, B.; Blum, L.; Perriat, P.; Tillement, O., Chem. Mater. 2005, 17: p. 1673

5- $\quad$ Chan, W. C. W.; Nie, S. M., Science 1998, 281: p. 201638

6- Whaley, S.R.; English, D.S.; Hu, E.L.; Barbara, P.F.; Belcher, A.M., Nature 2000, 405: p. 665 
7- $\quad$ Bruchez, M., Jr.; Moronne, M.; Gin, P.; Weiss, S.; Alivisatos, A.P., Science 1998, 281: p. 2013

8- Mitchell, G.P.; Mirkin, C.A.; Letsinger, R.L., J. Am. Chem. Soc. 1999, 121: p. 8122

9- $\quad$ Mednitz, I.L.; Uyeda, H.T.; Goldman, E.R.; Mattoussi, H., Nat. Mater. 2005, 4: p. 435

10- Hezinger, A.F.E.; Teßmar, J.; Göpferich, A., Eur. J. Pharma. Biopharma. 2008, 68: p. 1

11- Alivisatos A. P., Science 1996, 271: p. 933

12- $\quad$ http://www.evidenttech.com/products/evitags/overview.php

13- Qu, L.; Peng, Z. A.; Peng, X., Nano Lett. 2001, 1: p. 333

14- Ooba, H., AIST Today 2006, 6: p. 26

15- Wilcoxon, J. P.; Provenico, P. P., J. Phys. Chem. B 2005, 109: p. 13461

16- $\quad$ Zhang, S,; Yu, J.; Li, X.; Tian, W., Nanotechnology 2004, 15: p. 1108

17- Bruchez, M.; Moronne, M.; Gin, P.; Weiss, S.; Alivisatos, A. P., 281: p. 2013.

18- Gerion, D.; Pinaud, F.; Williams, S. C.; Parak, W. J.; Zanchet, D.; Weiss, S.; Alivisatos, A.P., J. Phys. Chem. B 2001, 105: p. 8861.

19- Guo, W.; Li, J. J.; Wang, Y. A.; Peng, X., J. Am. Chem. Soc. 2003, 125: p. 3901.

20- Larson, D. R.; Zipfel, W. R.; Williams, R. M.; Clark, S. W.; Bruchez, M. P.; Wise, F. W.; Webb, W.W., Science 2003, 300: p.1434.

21- Dubertret, B.; Skourides, P.; Norris, D. J.; Noireaux, V.; Brivanlou, A. H.; Libchaber, A., Science 2002, 298: p. 1759.

22- Mattoussi, H.; Mauro, J. M.; Goldman, E. R.; Green, T. M.; Anderson, G. P.; Sundar, V. C.; Bawendi, M. G., Phys. Status Solidi B 2001, 224: p. 277.

23- Sukhanova, A.; Venteo, L.; Devy, J.; Artemyev, M.; Oleinikov, V.; Pluot, M.; Nabiev, I., Lab. InVest. 2002, 82: p. 1259

24- Akerman, M. E.; Chan, P.; Laakkonen, S. N.; Bhatia, E.; Ruoslahti, E., PNAS 2002, 99: p. 12617

25- Kim, S.; Bawendi, M. G., J. Am. Chem. Soc. 2003, 125: p. 14652

26- Dubertret, B.; Skourides, P.; Norris, D. J.; Noireaux, V.; Brivanlou, A. H.; Libchaber, A., Science 2002, 298: p. 1759

27- Geissbuehler, I.; Hovius, R.; Martinez, K. L.; Adrian, M.; Thampi, K.; Ravindranathan, T.; Vogel, H., Angew. Chem., Int. Ed. 2005, 44: p. 1388

28- Wu, X.; Liu, H.; Liu, J.; Haley, K. N.; Treadway, J. A.; Larson, J. P.; Ge, N.; Peale, F.; Bruchez, M. P., Nat. Biotechnol. 2003, 21: p. 41 
29- Pellegrino, T.; Manna, L.; Kudera, K.; Liedl, T.; Koktysh, D.; Rogach, A. L.; Keller, S.; Radler, J.; Natile, G.; Parak, W.P., Nano Lett. 2004, 4: p. 704

30- Gao, X.; Cui, Y.; Levenson, R. M.; Chung, L. W.; Nie, S., Nat. Biotechnol. 2004, 22: p. 959

31- Kim, S. W.; Kim, S.; Tracy, J. B.; Jasanoff, A.; Bawendi, M. G., J. Am. Chem. Soc. 2005, 127: p. 4556

32- Bakueva, L.; Musikhin, S.; Hines, M. A.; Chang, T. W. F.; Tzolov, M.; Scholes, G. D.; Sargent, E. H., Appl. Phys. Lett. 2003, 82: p. 2895

33- Olsson, Y. K.; Chen, G.; Rapaport, R.; Fuchs, D. T.; Sundar, V. C.; Steckel, J. S.; Bawendi, M. G.; Aharoni, A.; Banin, U., Appl. Phys. Lett. 2004, 85: p. 4469

34- Caruso, F., Adv. Mater. 2001, 13: p. 11

35- Zhang, Y.; Luo, S.; Liu, S., Macromolecules 2005, 38: p. 9813

36- Galaev, I. Y.; Mattiasson, B., Trends Biotechnol. 1999, 17: p. 335

37- Heskins, M.; Guillet, J. E., J. Macromolecules Sci., Chem 1968, A2: p. 1441

38- Schild, H. G.; Tirrell, D. A., Langmuir 1991, 7: p. 665

39- Kohori, F.; Sakai, K.; Aoyagi, T.; Yokoyama, M.; Sakurai, Y.; Okano, T., J. Control. Rel. 1998, 55: p. 87

40- Sershen, S.R.; Westcott, S.L.; Halas, N.J.; West, J.L., J. Biomed. Mater. Res. 2000, 51: p. 293

41- Yuk, S.H.; Cho, S.H.; Lee, S.H., Macromolecules 1997, 30: p. 6856

42- $\quad$ Cammas, S.; Suzuki, K.; Sone, C.; Sakurai, Y.; Kataoka, K.; Okano, T., J. Control. Release 1997, 48: p. 157

43- Chung, J.E.; Yokoyama, M.; Suzuki, K.; Aoyagi, T.; Sakurai, Y.; Okano, T., Colloid. Surface. B 1997, 9: p. 37

44- $\quad$ Sershen, S. R.; Westcott, S. L.; Halas, N. J.; West, J. L., Appl. Phys. Lett. 2002, 80: p. 4609

45- Li, J.; Hong, X.; Liu Y.; Li, D.; Wang, Y.W.; Li, J.H.; Bai, Y.B.; Li, T.J., Adv. Mater. 2005, 17: p. 163

46- $\quad$ Li, J.; Liu, B.; Li, J., Langmuir 2006, 22: p. 528

47- Kuang, M.; Wang, D. Y.; Bao, H. B.; Gao, M. Y. Mohwald, H.; Jiang, M., Adv. Mater. 2005, 17: p. 267

48- Tsay, J. M.; Doose, S.; Pinaud, F.; Weiss, S., J. Phys. Chem. B 2005, 109: p. 1669

49- Sheng, W.; Kim, S.; Lee, J.; Kim, S. W.; Jensen, K.; Bawendi, M. G., Langmuir 2006, 22: p. 3782 
50- Decher, G., Science 1997, 277: p. 1232

51- Decher, G.; Hong, J. D., Makromol. Chem. Macromol. Symp. 1991, 46: p. 321

52- Liang, Z.; Dzienis, K. L.; Xu, J.; Wang, Q., Adv. Funct. Mater. 2006, 16: p. 542

53- $\quad$ Yang, P.; Li, C. L.; Murase, N., Langmuir 2005, 21: p. 8913

54- Li, F.B.; Albery, W.J., Adv. Mater. 1992, 4: p.673

55- Mamedov, A. A.; Belov, A.; Giersig, M.; Mamedova, N. N.; Kotov, N. A., J. Am. Chem. Soc. 2001, 123: p. 7738

56- Zhang, H.; Zhou, Z.; Liu, K.; Wang, R.; Yang, B., J. Mater. Chem. 2003, 13: p. 1356

57- $\quad$ Tang, Z.; Wang, Y.; Kotov, N. A., Langmuir 2002, 18: p. 7035

58- $\quad$ Lesser, C.; Gao, M.; Kirstein, S., Mater. Sci. Eng., C 1999, 8-9: p. 159

59- Zhou, D.; Bruckbauer, A.; Abell, C.; Klenerman, D.; Kang, D.J., Adv. Mater. 2005, 17: p. 1243

60- Jaffar, S.; Nam, K. T.; Khademhosseini, A.; Xing, J.; Langer, R. S.; Belcher, A. M., Nano Lett. 2004, 4: p. 1421

61- Gorelikov, I.; Kumacheva, E., Chem. Mater. 2004, 16: p. 4122

62- Schneider, G.; Decher, G., Nano Lett. 2006, 6: p. 530

63- Lowman, G. M.; Nelson, S. L.; Graves, S. M.; Strouse, G. F.; Buratto, S. K., Langmuir 2004, 20: p. 2057

64- Colvin, V. L; Schlamp, M.C.; Alivisatos, A.P., Nature 1994, 370: p. 534

65- Yang, X.; Zhang, Y., Langmuir 2004, 20: p. 6071

66- Li, Y.; Liu, E.C.Y.; Pickett, N.; Skabara, P. J.; Cummins, S.S.; Ryley, S.; Sutherland, A.J.; O’Brien, P., J. Mater. Chem. 2005, 15: p. 1238

67- Esteves, A.C.C.; Barros-Timmons, A.M.V.; Monteiro, T.; Trindade, T., J. Nanosci. Nanotechnol. 2005, 5: p. 766

68- $\quad$ Peres, M.; Costa, L.C.; Neves, A.; Soares, M.J.; Monteiro, T.; Esteves, A.C.; BarrosTimmons, A.; Trindade, T.; Kholkin, A.; Alves, E., Nanotechnology 2005, 16: p. 1969

69- Becerra, L.R.; Murray, C.B.; Griffin, R.G.; Bawendi, M.G., J. Chem. Phys. 1994, 100: p. 3297

70- Bowen, K.J.E.; Colvin, V.L.; Alivisatos, A.P., J. Phys. Chem. 1994, 98: p. 4109

71- Sill, K. and Emrick, T., Chem. Mater. 2004, 16: p. 1240

72- $\quad$ Zhang, H.; Wang, C.; Li, M.; Ji, X.; Zhang, J.; Yang, B., Chem. Mater. 2005, 17: p. 4783

73- Bao, H.; Gong, Y.; Li, Z.; Gao, M. Y., Chem. Mater. 2004, 16: p. 3853 
74- Gao, M.Y.; Kirstein, S.; Mohwald, H.; Rogach, A.L.; Kornowski, A.; Eychmuller, A.; Weller, H., J. Phys. Chem. B 1998, 102: p. 8360

75- Li, D.; Jones, G.L.; Dunlap, J.R.; Hua, F.; Zhao, B., Langmuir 2006, 22: p. 3344

76- Jordan, R.; Graft, K.; Riegler, H.; Unger, K. K., Chem. Commun. 1996, 9: p. 1025

77- de Boer, B.; Simon, H. K.; Werts, M. P.L.; van der Vegte, E. W.; Hadziioannou, G., Macromolecules 2000, 33: p. 349

78- Reddy, S.K.; Sebra, R.P.; Anseth, K.S.; Bowman, C.N., J. Polym. Sci. A, Polym. Chem. 2005, 43: p. 2134

79- Luo, N.; Hutchison, J. B.; Kristi S. Anseth, K. S.; Bowman, C. N., Macromolecules 2002, 35: p. 2487

80- Ohno, K.; Morinaga, T.; Koh, K.; Tsujii, Y.; Fukuda, T., Macromolecules 2005, 38: p. 2137

81- Bai, J.; Qiu, K. Y.; Wei, Y., Polym. Int. 2003, 52: p. 853

82- Ohno, K.; Koh, K.; Tsujii, Y.; Fukuda, T., Angew. Chem., Int. Ed. 2003, 42: p. 2751

83- Farmer, S.C.; Patten, T.E., Chem. Mater. 2001, 13: p. 3920

84- Vestal, C.R.; Zhang, Z.J., J. Am. Chem. Soc. 2002, 124: p. 14312

85 - Matsuno, R.; Yamamoto, K.; Otsuka, H.; Takahara, A., Macromolecules 2004, 37: p. 2203

86- Bekiari, V.; Pagonis, K.; Bokias, G.; Lianos, P., Langmuir, 2004, 20: p.7972

87- Zhao, H.; Douglas, E.P.; Harrison, B.S.; Schanze, K.S., Langmuir 2001, 17: p. 8428.

88- $\quad$ Simmons, B.A.; Sichu, L.; Vijay, J.T.; McPherson, G.L.; Bose, A.; Zhou, W.; He, J., Nano Lett. 2002, 2: p. 263

89- Jones, C.D.; Serpe, M.J.; Schroeder, L.; Lyon, A., J. Am. Chem. Soc. 2003, 125: p. 5292

90- Xulu, P. M.; Filipsei, G.; Zrinyl, M., Macromolecules 2000, 33: p. 1716.

91- Moffitt, M.; McMahon, L.; Pessel, V.; Eisenberg, A., Chem. Mater. 1995, 7: p. 1185

92- Duxin, N.; Liu, F.; Vali, H.; Eisenberg, A., J. Am. Chem. Soc. 2005, 127: p. 10063

93- Moffitt, M.; Vali, H.; Eisenberg, A., Chem. Mater. 1998, 10: p. 1021

94- Wang, D.; Yaan, C.; Xintong, Z.; Zhiqiang, L.; Xinming, Q.; Xin, A.; Fengqi, L.; Dejun, W.; Yubai, B.; Tiejin, L.; Tang, X., Chem. Mater. 1999, 11: p. 392

95- Zhao, H.; Douglas, E.P., Chem. Mater. 2002, 14: p. 1418

96- Liu, T.; Burger, C.; Chu, B., Prog. Polym. Sci. 2002, 28: p. 5

97- Chu, Y.C.; Wang, C.C.; Chen, C.Y., Nanotechnology 2005, 16: p. 58 
98- Pardo-Yissar, V.; Gabai, R.; Shipway, A.N.; Bourenko, T.; Willner, I., Adv. Mater. 2001, 13: p. 1320

99- Zhang, J.; Coombs, N.; Kumacheva, E.; Lin, Y.; Sargent, E.H., Adv. Mater. 2002, 14: p. 1756

100- Tomczak, N.; Janczewski, D.; Han, M.-Y.; Vancso, G.J., Prog Polym Sci, 2009. 34: p. 393. 


\section{Chapter 3}

\section{Characterization methods of quantum dots and quantum dot/polymer assemblies}

This chapter describes some of the microscopy and spectroscopy techniques used in this thesis for the characterization of QDs. In addition to atomic force microscopy and confocal microscopy, combined microscopy techniques for studying $Q D$ s and $Q D$-bearing systems are explained. The spectroscopic techniques which are mentioned in this chapter include time correlated single photon counting and fluorescence correlation spectroscopy. 



\subsection{Introduction}

It is of great importance to obtain detailed information on the structure of QD surfaces due to their strong influence on the colloidal and optical properties of the QDs. Surface characterization methods are required for the monitoring of colloidal and optical properties each time a chemical modification is made. Colloidal properties such as particle size, size distribution and particle aggregation are monitored by transmission electron microscopy (TEM), atomic force microscopy (AFM), light scattering, chromatography, and electrophoresis [1]. Given the typical values of the relevant energy levels in QDs, the photophysical characterization methods usually involve UV/visible absorption and fluorescence emission spectroscopies.

Monitoring the excited states of QDs proves useful for surface characterization of QDs, since luminescence behavior is influenced by the polarity, dielectric constant, or the presence of quenchers in the environment of the excited species [2]. In this context, timeresolved luminescence spectroscopy and time-resolved fluorescence lifetime measurements give information about the charge carrier dynamics on the QD surface. Simple quenching experiments with a number of quenchers with different redox potentials can be used to detect the energy levels of surface traps.

In this chapter, some of the important microscopic and spectroscopic characterization methods are described.

\subsection{Microscopy techniques}

With recent advances in nanotechnology, the conventional microscopy has been replaced by more powerful detection methods with single molecule sensitivity. Single molecule detection enables determination of dynamic as well as static heterogeneities that are masked in ensemble-averaging methods.

\subsubsection{Atomic force microscopy}

Atomic force microscope (AFM) provides high spatial resolution, three-dimensional topographical information on sample surfaces in air and in aqueous environment, and therefore it is a powerful tool for imaging, characterization and manipulation of matter at the nanoscale [3]. Along with the topographic image, other properties, such as elasticity or adhesion force can be probed and mapped with this technique [4].

An AFM consists of a cantilever with a sharp tip (probe) at its end, which is used to scan the specimen surface [5]. When the tip is brought into proximity of a sample surface, 
intermolecular forces (e.g. electrostatic forces, Van der Waals forces, etc.) between the tip and the sample result in a deflection of the cantilever (Figure 3.1.). Typically, the deflection is measured using a laser spot reflected from the top of the cantilever and directed onto a photodiode array.

A feedback mechanism is employed to adjust the deflection to maintain a constant force between the tip and the sample during imaging. Typically, the sample is mounted on a piezoelectric tube, which can move the sample in the $z$ direction to maintain a constant force, and the $x$ and $y$ directions for scanning the sample.

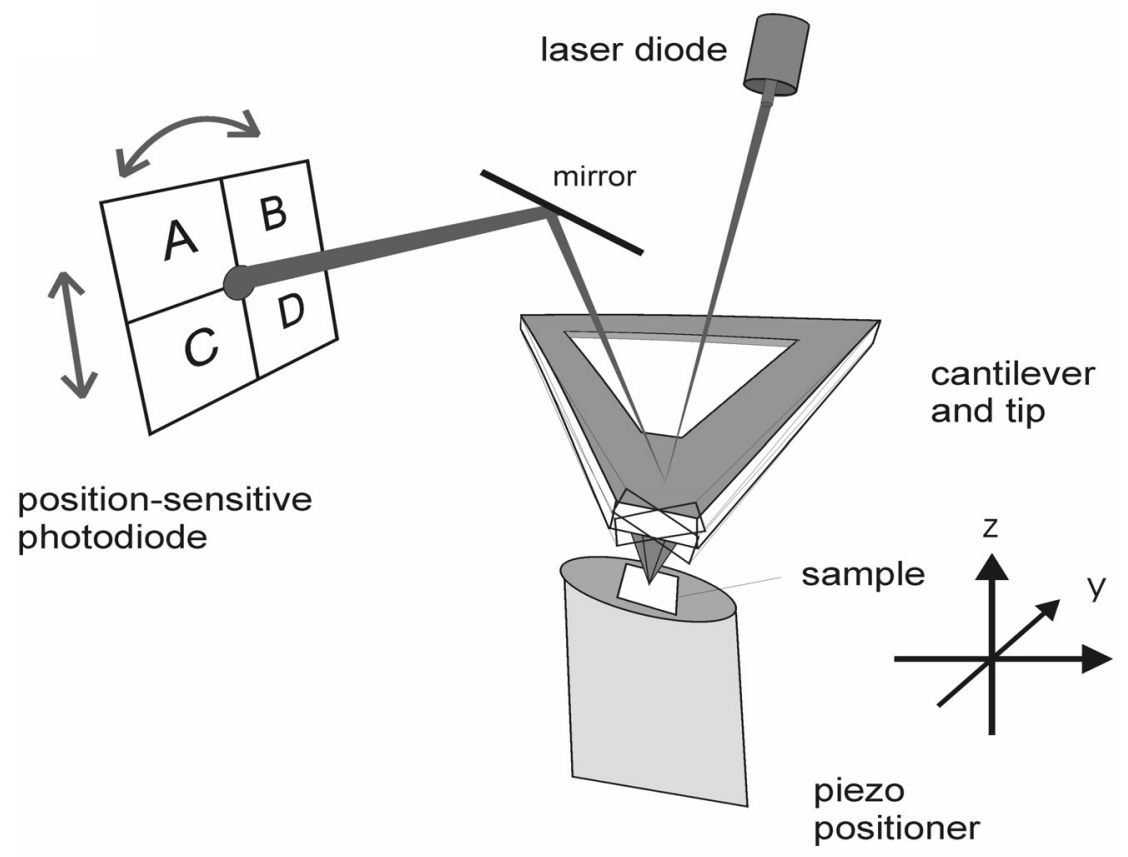

Figure 3.1. Schematic presentation of an atomic force microscope (the components are not drawn to scale). The devices for data acquisition and signal processing have been omitted for clarity. The image was taken from [4].

The interactions between the AFM tip and the sample can be monitored by measuring the cantilever deflection as a function of the scanner movement along the z-axis. Figure 3.2 shows the cantilever behavior as it approaches to, and retracts from, the sample surface. Initially there is a weak repulsive force (1) until the tip gets in contact with the surface (2). Further movement towards the surface results in bending of the cantilever (3) due to stiffness of the surface. During retraction, the cantilever remains at the surface (4) until it overcomes the adhesive forces (5) and detaches from the surface. 


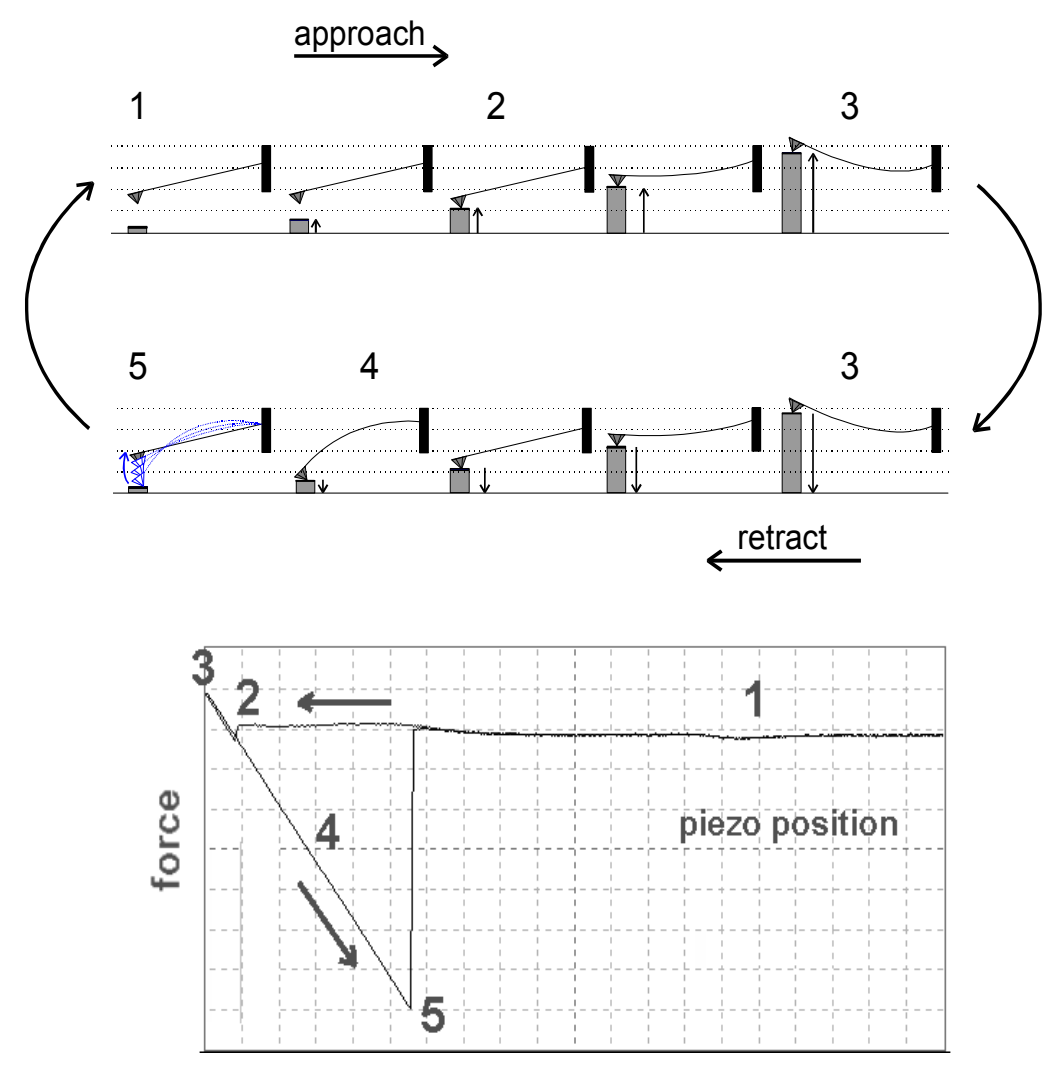

Figure 3.2. i) Schematic presentation of the cantilever deflection versus scanner movement when approaching to and retracting from the surface. ii) Schematic force-distance plot. (1) is the non-contact region. After initial contact with the surface (2) the cantilever bends due to further movement of the piezo (3). The cantilever remains at the surface (4) until it overcomes the adhesive forces (5) and completely detaches from the surface. This image was taken from [4].

An AFM can be operated in a number of modes, depending on the application. For imaging purposes, the primary modes of operation are the contact and the tapping modes. In the contact mode, the force between the tip and the surface is kept constant during rasterscanning by maintaining a constant cantilever deflection. In the tapping mode, the cantilever is vibrating such that it comes in brief contact with the sample during each cycle, and then enough restoring force is provided by the cantilever spring to separate the tip from the sample.

In addition to the determination of surface topography, AFM has been used as a tool for the determination of the elastic properties of polymer surfaces [6-8] via indentation experiments. For instance, AFM in the force spectroscopy mode has been used to study the mechanical properties of thin polymer layers [9], polyelectrolyte multi layers [10], as well as 
the viscoelastic and plastic properties of amorphous polymer surfaces as a function of temperature across the glass transition temperature $(\mathrm{Tg})$ [11]. Due to the small dimension of the AFM probe, related nanoindentation experiments provided information on the nanomechanical properties, e.g. on the Young's modulus, of these surfaces with unprecedented lateral resolution. [12-14].

The indentation experiments involve acquisition of deflection-distance curves and conversion of the cantilever deflection and scanner extension data into force and indentation, respectively. A representative curve is shown in Figure 3.3. Either the approach or the retraction curve can be used for determination of the mechanical properties of the samples. A hard substrate with 'zero indentation' is used as a reference [15].

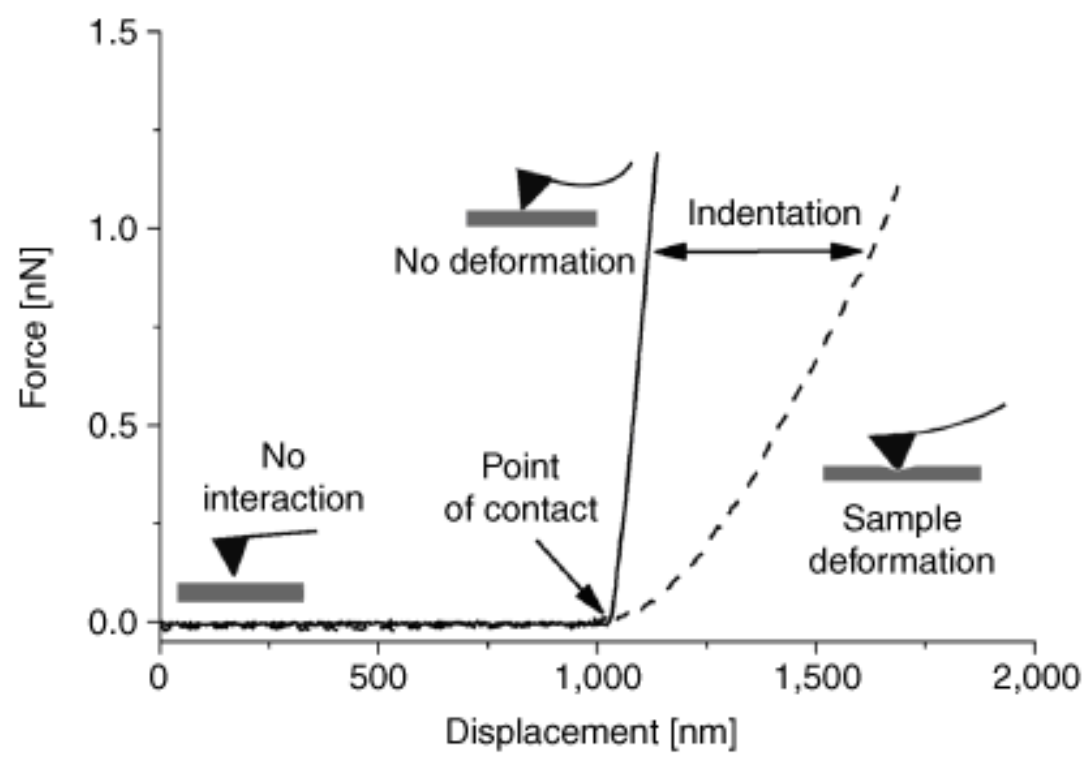

Figure 3.3. Illustration of an indentation experiment and the corresponding force displacement curves obtained for a hard substrate (solid) and a softer sample (dash). The contact regions are used for the determination of mechanical properties. The difference in the displacement between the hard substrate and the soft material corresponds to the indentation of the tip into the sample. This image was taken from [15].

The versatility of AFM technique enables studying materials' properties under different environments (air, or liquid environment) and at different temperatures. 


\subsubsection{Confocal microscopy}

Confocal laser scanning fluorescence microscopy is a widely used technique in life sciences for obtaining high-resolution optical images with depth selectivity [16, 17]. By using a process called optical sectioning, in-focus images from the selected depths are acquired. During this process out-of-focus light is rejected, which improves the signal to noise ratio [17]. The schematic presentation of a confocal setup is shown in Figure 3.4.

Light source

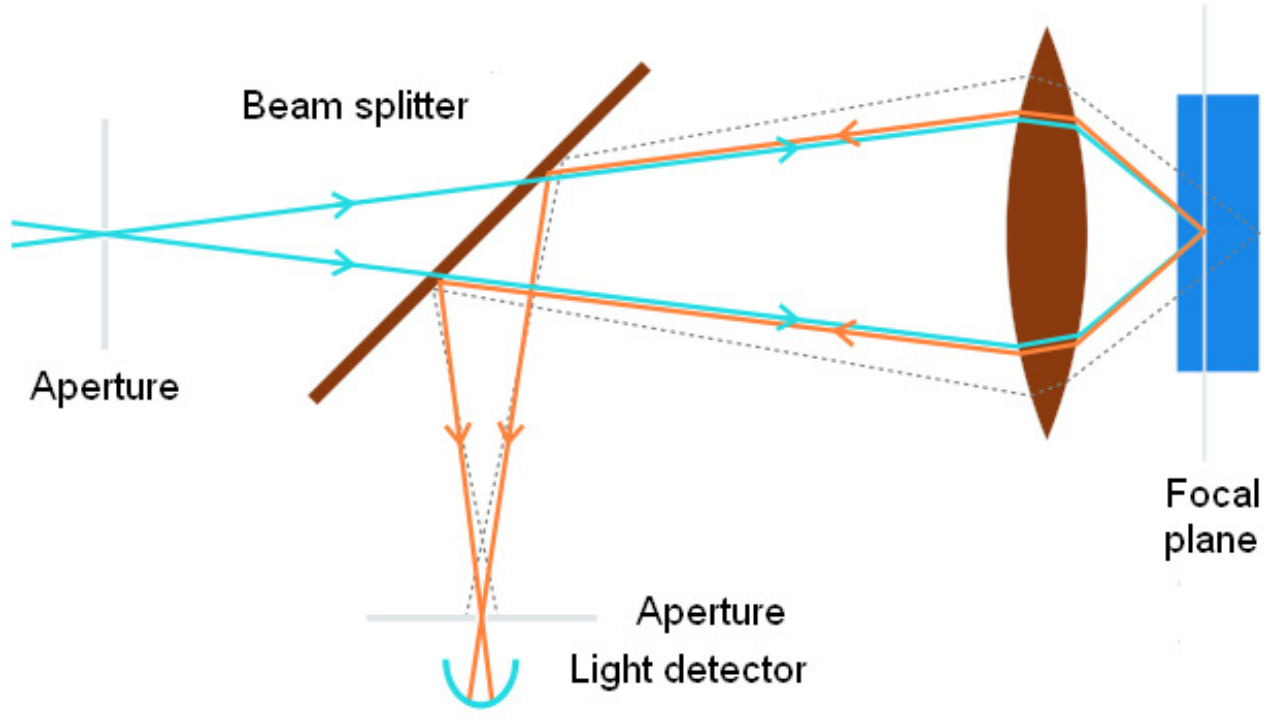

Figure 3.4. Schematic presentation of a confocal microscope. Blue: excitation path; red: detection path. The excitation beam is focused to a diffraction-limited volume. The emitted fluorescence is collected by the objective and directed to the detector by using a beamsplitter. The image was adapted from [18].

In a confocal laser scanning microscope, the laser beam passes through an aperture and then is focused by an objective lens to a diffraction-limited focal volume. The objective lens re-collects the emitted fluorescence as well as scattered and reflected laser light. A beamsplitter sends the light to the detection apparatus, which is equipped with filters that selectively allow passing only the fluorescence. After passing through a pinhole, the fluorescence light intensity is detected by photomultiplier tubes (PMTs), or avalanche photodiodes (APDs).

There have been a number of investigations of individual QDs immobilized on surfaces with confocal microscopy. For instance, Nirmal et al. for the first time reported blinking in the emission of single QDs (Figure 3.5) using a scanning stage confocal microscope, equipped with a CCD camera [19]. More recent works supported the 
interpretation that blinking is caused by surface defects that can be altered by surface chemistries and buffer conditions [20-22].
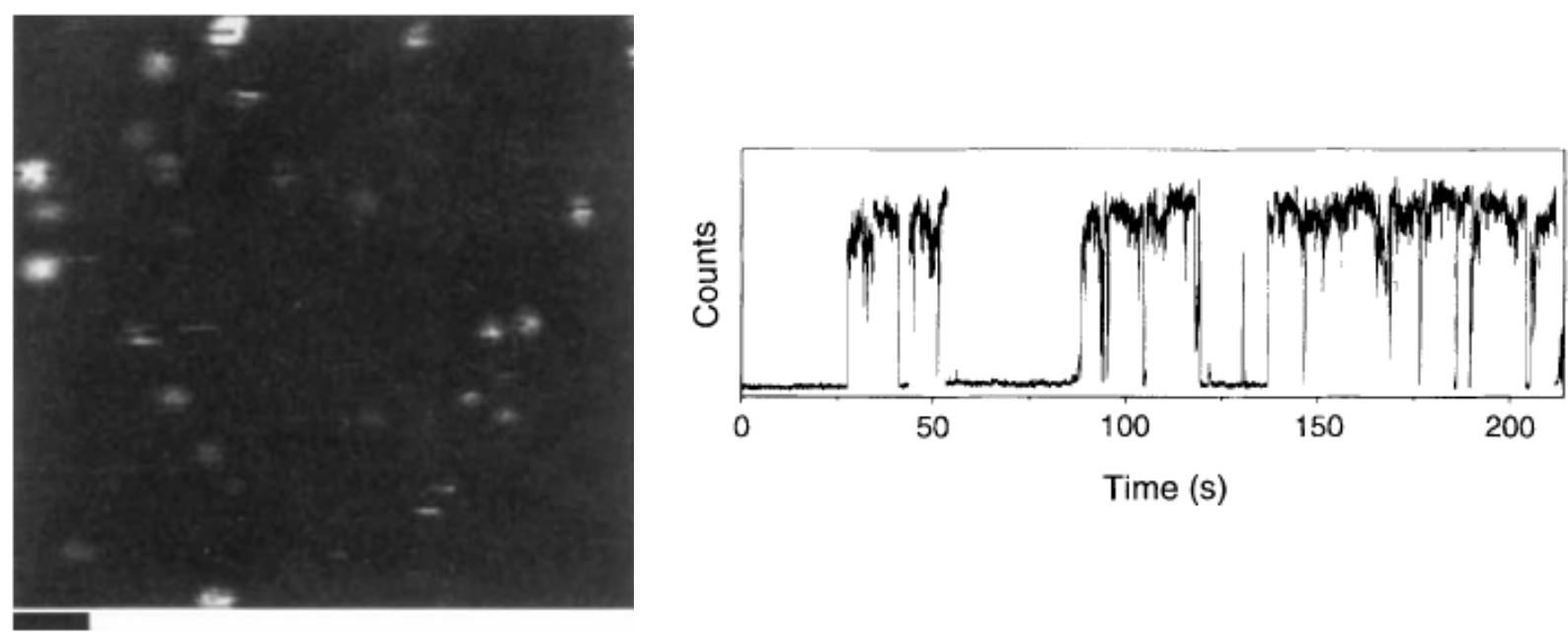

$1 \mu \mathrm{m}$

Figure 3.5. Left: Confocal microscopy image of single CdSe QDs embedded in a thin polyvinylbutyral film acquired by raster scanning the sample across a diffraction limited laser spot $(532 \mathrm{~nm})$. Field of view: $8 \mathrm{x} 8 \mu \mathrm{m}$. Right: Fluorescence intensity versus time trace of a single QD. This image was taken from [19].

\subsubsection{Combination of different microscopy techniques}

AFM and optical microscopy have both experienced significant advancements in recent years [23]. For instance, several improvements enabled overcoming the diffraction limit in optical microscopy [24]. Similarly, AFM has been applied for the determination of electrostatic properties of biomolecules [25], and for the measurements of mechanical properties of such molecules [26, 27]. Combining these two techniques has opened up many exciting possibilities in biophysics, materials science and bionanotechnology by providing nanoscale resolution and quantitative force measurements as well as spectral characterization $[23,28,29]$. Owen et al. have reported on simultaneous AFM and confocal imaging of $\mathrm{CdSe} / \mathrm{ZnS}$ core/shell QDs of $3.5 \mathrm{~nm}$ core size with high spatial resolution [23]. Lately, Ebenstein et al. showed how to utilize QDs as contrast agents for both AFM topography and fluorescence imaging with a combined setup [30]. In addition, Wang et al. employed QDs as bifunctional cell-surface markers for both AFM and fluorescence microscopy for quantitative 
investigation of the distribution and expression of membrane proteins on cell surface using simultaneous fluorescence and topography mapping [31]. In a study reported by Doak et al. a combined AFM and confocal microscopy setup was used for simultaneous detection of structural and fluorescent properties of PNT2 cells, a cell line derived from human prostate epithelial cells, labeled with QDs [28] (Figure 3.6). The topographic features of the cells were visualized by AFM and their molecular make-up was investigated using confocal microscopy.
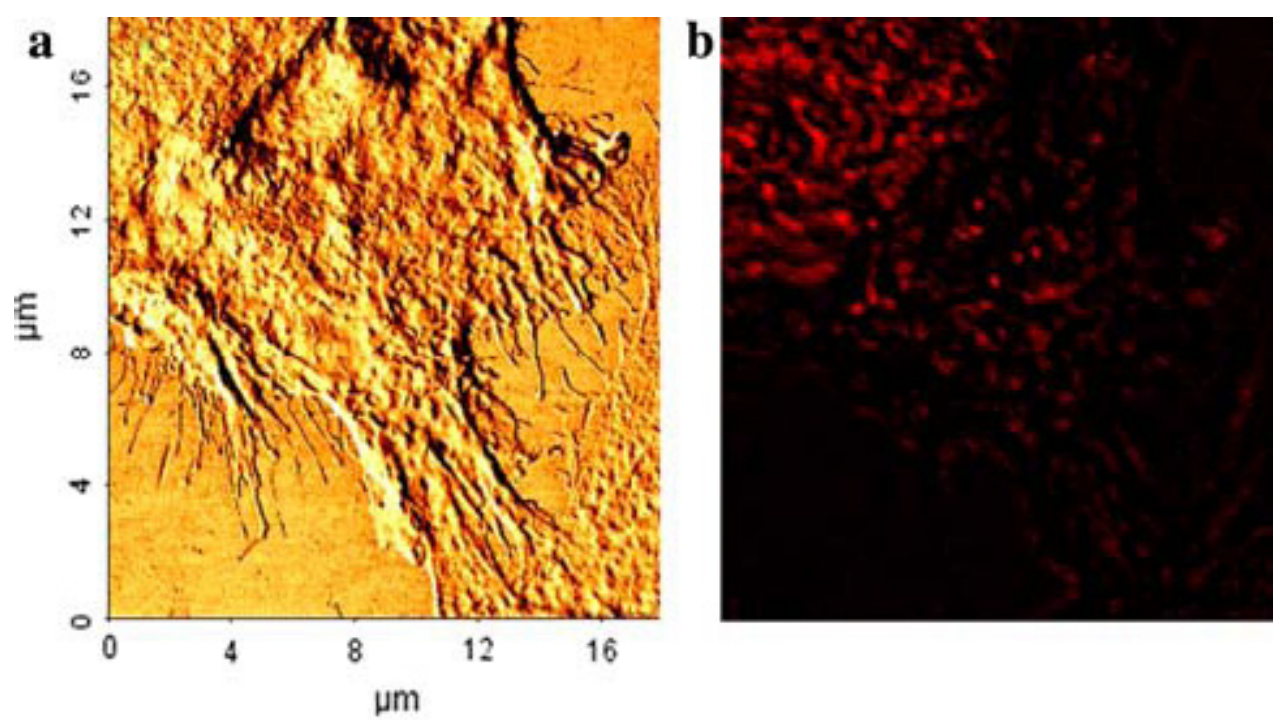

Figure 3.6.a) AFM image of PNT2 cells with QD labelled F-actin, and b) corresponding confocal microscopy image of the same region. This image was taken from [28].

The combined microscopy techniques mentioned here have the potential to be applied to a variety of samples, including living cells, and can be coupled with AFM-based force measurements for further biophysical analysis. Therefore, these techniques will not only provide significant advances in imaging, but will also enable the characterization of structural constituents, the mechanical properties and structure-function relationships of biological molecules at the single molecule level.

\subsection{Spectroscopy techniques}

The key to understanding of the optical and colloidal properties of QDs is to study their interactions with light. Fluorescence and UV/visible absorption spectroscopy are two commonly used techniques for basic optical characterization [32]. The techniques have become very popular because of their sensitivity to changes in the structural and 
photophysical properties of QDs. Steady-state or time resolved emission or absorption can be measured.

Ultrafast spectroscopy provides essential information on the mechanistic and kinetic details of chemical and physical events that occur on the timescale of tens of femtoseconds to several nanoseconds. Such events are initiated by an ultrafast laser pulse (pump) and the photophysical and photochemical events are probed by another ultrafast laser pulse (probe). With sub femtoseconds pulse durations, it is possible to access the dynamics of the internal conversion, intramolecular vibrational relaxation and many other processes, which steer the energy into different reaction pathways [33] [34].

\subsubsection{Time correlated single photon counting (TCSPC)}

TCSPC is a technique used for recording low-level light signals with picosecond time resolution. It is based on the excitation with a periodic light source and measuring the arrival times of individual fluorescence photons [33]. The method makes use of the fact that for low light level and high excitation repetition rates, the probability of detecting one photon between excitation light pulses is much lower than one. The time between the excitation light pulse and the detected fluorescence photon is measured. This time corresponds to the excited state lifetime of the investigated species. Histogram of the photon arrival times corresponds to the time-averaged luminescence decay curves from which an average excited state lifetime can be obtained.

TCSPC has been extensively used for the investigation of carrier dynamics in excited QDs [35-37].

\subsubsection{Fluorescence correlation spectroscopy (FCS)}

Fluorescence correlation spectroscopy is a sensitive single molecule detection method with a wide range of applications in different fields [38-41]. It is based on the monitoring of the fluctuating fluorescence signal of the emitter molecule as it passes through the confocal volume [42]. Autocorrelation function is then calculated according to Eq. (3.1):

$$
G(\tau)=1+\frac{\langle\delta I(t) \delta I(t+\tau)\rangle}{\langle I(t)\rangle^{2}}
$$

In this equation, $t$ is the time variable corresponding to the time frame over which the intensity signal $I$ is defined, while $\tau$ is the delay variable corresponding to the time frame, 
over which the relative fluctuations of $I$ are measured, and the autocorrelation function $G$ is defined. The fluorescence fluctuation around the average intensity, $\delta I(t)$, is monitored and correlated to the function obtained at time $t+\tau$ to generate the autocorrelation function [42]. The autocorrelation function of the fluctuations resulting from the diffusion of the molecules through the focal volume is used to obtain the diffusion coefficient by setting Eq. (3.1) equal to the autocorrelation function for a 3D Gaussian excitation volume shown in Eq. (3.2):

$$
G(\tau)=1+\frac{1}{N}\left(1+\frac{\tau}{\tau_{D}}\right)^{-1}\left(1+S^{2} \frac{\tau}{\tau_{D}}\right)^{-\frac{1}{2}}
$$

In the equation above, $N$ represents the number of fluorophores in the confocal volume at any time, $\tau_{D}$ is diffusion time of the molecule through the confocal volume and $S$ is the structure parameter, which is the ratio of axial $\left(\omega_{z}\right)$ and radial $\left(\omega_{r}\right)$ distances at which the intensity of the Gaussian excitation falls to $\mathrm{e}^{-2}$ of its maximum. The inverse relation between the autocorrelation signal and the number of fluorophores enables measurements in the nanomolar range.

FCS performed on diffusing molecules has single molecule sensitivity and is compatible with high throughput analysis [43-45]. Despite using nanomolar concentrations, it can provide evidence on distributions of characteristic time constants and sample heterogeneities [46].

FCS has been used in a wide range of biochemical assays [41]. It has been applied to monitor conformational fluctuations of biopolymers, detect binding events between macromolecules and their ligands, investigate photophysical properties of organic dyes and fluorescent proteins, or look at diffusion properties of labeled macromolecules in vitro and in vivo.

Doose et al. reported use of FCS for characterization of the photophysical and colloidal properties of different water-stable QDs [46]. They have reported on the brightness per particle, saturation intensity, blinking, and hydrodynamic radii for different QDs and demonstrated that variations in the FCS amplitude reflected the sample inhomogeneities. More detailed discussions on the advances, challenges and opportunities of using FCS for characterization of QDs can be found in [47].

The microscopy and spectroscopy techniques mentioned here are useful tools for characterization of QDs and QD/polymer hybrid structures in terms of their colloidal and 
optical properties down to single molecule level. Combining different microscopy and spectroscopy techniques will enable simultaneous characterization of different properties such as morphology, optical and colloidal stability, etc.

\subsection{References}

1. Doose, S.; Tsay, J.M.; Pinaud, F.; Weiss, S., Anal Chem, 2005. 77: p. 2235.

2. Thomas, J.K., J Phys Chem, 1987. 91: p. 267.

3. Binnig, G.; Quate, C.F.; Gerber, C., Phys Rev Lett, 1986. 56: p. 930.

4. Schönherr, H., From functional group ensembles to single molecules: scanning force microscopy of supramolecular and polymeric systems. $\mathrm{Ph}$ D Thesis 1999, University of Twente: Enschede.

5. Peterson, C.A.; Sarid, D., Kirk-Othmer Encyclopedia of Chemical Technology: Atomic Force Microscopy. Vol. 3. 2007, New Jersey: Wiley.

6. $\quad$ Matzelle, T.R.; Geuskens, G.; Kruse, N., Macromolecules, 2003. 36: p. 2926.

7. $\quad$ Briscoe, B.; Fiori, L.; Pelillo, E., J Phys D, 1998. 31: p. 2395.

8. Butt, H.-J.; Cappella, B.; Kappl, M., Surf Sci Rep, 2005. 59: p. 1.

9. Dimitriadis, E.K.; Horkay, F.; Maresca, J.; Kachar, B.; Chadwick, R. S., Biophys J, 2002. 82: p. 2798.

10. Mermut, O.; Lefebre, J.; Gray, D. G.; Barrett, C. J., Macromolecules, 2003. 36: p. 8819.

11. Cappella, B.; Kaliappan, S.K.; Sturm, H., Macromolecules, 2005. 38: p. 1874.

12. Chizhik, S.A.; Huang, Z.; Gorbunov, V. V.; Myshkin, N. K.; Tsukruk, V. V., Langmuir, 1998. 14: p. 2606.

13. Reynaud, C.; Sommer, F.; Quet, C.; El Bounia, M.; Duc, T. M., Surf Interface Anal, 2000. 30: p. 185.

14. Raghavan, D.; Gu, X.; Nguyen, T.; VanLandingham, M. R.; Karim, A., Macromolecules, 2000. 33: p. 2573.

15. Puttini, S.; Lekka, M.; Dorchies, O.M.; Saugy, D.; Incitti, T.; Ruegg, U.T.; Bozzoni, I.; Kulik, A.J.; Mermod, N., Mol Theraph, 2008. 17: p. 19.

16. Fellers, T.J.; Davidson, M.W., Introduction to Confocal Microscopy. 2007, Olympus Fluoview Resource Center.

17. Pawley, J.B., Handbook of Biological Confocal Microscopy. 2006, Berlin: Springer.

18. Wright, S.J.; Wright, D.J., Methods Cell Biol, 2002. 70: p. 1. 
19. Nirmal, M.; Dabbousi, B. O.; Bawendi, M. G.; Macklin, J. J.; Trautman, J.K.; Harris, T. D.; Brus, L. E., Nature, 1996. 383: p. 802.

20. Hohng, S.; Ha, T. J., J Am Chem Soc, 2004. 126: p. 1324.

21. Kuno, M.; Fromm, D. P.; Hamann, H. F.; Gallagher, A.; Nesbitt, D. J., J Chem Phys, 2000. 112: p. 3117.

22. Shimizu, K.T.; Neuhauser, R. G.; Leatherdale, C. A.; Empedocles, S. A.; Woo, W. K.; Bawendi, M. G., Phys Rev B, 2001. 6320: p. U5395.

23. Owen, R.J.; Heyes, C.D.; Knebel, D.; Rocker, C.; Nienhaus, G.U., Biopolymers, 2006. 82: p. 410.

24. Hell, S.W., Nat Biotechnol, 2003. 21: p. 1347.

25. Muler, D.J.; Anderson, K., Trends Biotechnol, 2002. 20: p. S45.

26. Oesterhelt, F.; Oesterhelt, D.; Pfeiffer, M.; Engel, A.; Gaub, H. E.; Muller, D. J., Science, 2000. 288: p. 143.

27. Fernandez, J.M.; Li, H., Science, 2004. 303: p. 1674.

28. Doak, S.H.; Rogers, D.; Jones, B.; Francis, L.; Conlan, R.S.; Wright, C., Histochem Cell Biol, 2008. 130: p. 909.

29. Ebenstein, Y.; Mokari, T.; Banin, U., Appl Phys Lett, 2002. 80: p. 4033.

30. Ebenstein, Y.; Gassman, N.; Kim, S.; Weiss, S., J Mol Recog, 2009. 22: p. 397.

31. Wang, Y.; Chen, Y.; Cai, J.; Zhong, L., Ultramicroscopy, 2009. 109: p. 268.

32. Royer, C.A., Biophys J, 1995. 68: p. 1191.

33. Lakowicz, J.R., Principles of fluorescence spectroscopy. 2006, New York, NY: Springer.

34. Klimov, V.I., Annu Rev Phys Chem, 2007. 58: p. 635.

35. Yamada, T.; Goushi, K.; Xu, X.; Otomo, A., Thin Solid Films, 2008. 517: p. 1507.

36. Hong, M.; Guo-Hong, M.; Wen-Jun, W.; Xue-Xi, G.; Hong-Liang, M., Chinese Phys B, 2008. 17: p. 1280.

37. Nikolaev, I.S.; Lodahl, P.; van Driel, A.F.; Koenderink, A.F.; Vos, W.L., Phys Rev B, 2007. 75: p. 115302.

38. Kinjo, M., Anal Chim Acta, 1998. 365: p. 43.

39. Schwille, P., Cell Biochem Biopyhs, 2001. 34: p. 383.

40. Sengupta, P.; Garai, K.; Balaji, J.; Periasamy, N.; Maiti, S., Biophys J, 2003. 84: p. 1977.

41. Hess, S.T.; Huang, S.; Heikal, A.A.; Webb, W.W., Biochemistry, 2002. 41: p. 697.

42. Magde, D.; Elson, E.; Webb, W.W., Phys Rev Lett, 1972. 29: p. 705. 
43. Zhang, P.; Li, L.; Dong, C.; Qian, H.; Ren, J., Anal Chim Acta, 2005. 546: p. 46.

44. Akcakir, O.; Therrien, J.; Belomoin, G.; Barry, N.; Muller, J.D.; Gratton, E.; Nayfeh, M., Appl Phys Lett, 2000. 76: p. 1857.

45. Pellegrino, T.; Manna, L.; Kudera, S.; Liedl, T.; Koktysh, D.; Rogach, A.L.; Keller, S.; Rädler, J.; Natile, G.; Parak, W.J., Nano Lett, 2004. 4: p. 703.

46. Doose, S.; Tsay, J.M.; Pinaud, F.; Weiss, S., Anal Chem, 2005. 77: p. 2235.

47. Heuff, R.F.; Swift, J.L.; Cramb, D.T., Phys Chem Chem Phys, 2007. 9: p. 1870. 


\section{Chapter 4}

\section{Probing the morphology and nano-scale mechanics of single poly(N-isopropylacrylamide) microparticles across the lower critical solution temperature by atomic force microscopy*}

This chapter discusses the morphology and nano-mechanical properties of individual, isolated, PNIPAM microgel particles at the silicon/air, and silicon/water interfaces, below and above the PNIPAM volume phase transition temperature (VPTT) using atomic force microscopy. The elastic modulus calculated from force-indentation curves obtained for each individual microgel sphere reveals that the stiffness of the hydrogel's surface decreases by two orders of magnitude upon swelling in water. Additionally, the modulus of the PNIPAM spheres in water increases by one order of magnitude when crossing the VPTT indicating a more compact chain packing at the particle surface. A thorough characterization of the microparticles was necessary in view of subsequent sections in this thesis, focusing on QD/PNIPAM hybrid structures.

\footnotetext{
" Parts of this chapter have been published in: Tagit,O., Tomczak, N., Vancso, G.J. Probing the Morphology and Nanoscale Mechanics of Single Poly(N-isopropylacrylamide) Microgels Across the Lower-Critical-Solution Temperature by Atomic Force Microscopy Small 4119 - 126 (2008)
} 


\subsection{Introduction}

Responsive polymer hydrogels display swelling and shrinking behavior strongly influenced by an external stimulus [1] such as temperature [2], ionic strength [3], $\mathrm{pH}$ [4], light [5], or electric field [6]. These materials, due to their reversible changes in optical, mechanical or electronic properties in response to changes in their environment [7] are often referred to as "smart" and have found numerous applications in catalysis [8], chemical separation [9], in biology and medicine [10] for tissue engineering [11], controlled drug delivery [12], biological sensors [13], and biomolecule immobilization [14].

Poly(N-isopropylacrylamide) (PNIPAM) [15] is one of the best studied thermoresponsive polymers exhibiting a lower critical solution temperature (LCST), above which the solubility of the polymer in water decreases dramatically and the polymer phaseseparates. This transition (around $32^{\circ} \mathrm{C}$ for PNIPAM [16]) manifests itself as e.g. a sharp decrease in the microgel volume. For mildly crosslinked microgel systems a reversible volume-volume phase transition (VPT) is therefore observed, which usually occurs at the same critical temperature (i.e., LCST) as that of the uncrosslinked systems.

Recently, there has been growing interest in the fabrication of responsive organic/inorganic hybrid nanomaterials [17]. In this context, immobilization of metal [18-22] or semiconductor nanoparticles [23-25] in stimulus-responsive polymer hydrogels and microgels provides novel platforms with unique combination of properties for applications in chemical sensing or biodiagnostics. However, prior to realizing applications of these platforms it is necessary to study and understand the factors that affect their VPTT, as well as the shape and physical properties of the microparticles. The associated uptake and release of water from the hydrogel's interior influence the macroscopic dimensions of the polymers as well as the polymer chain packing at the molecular level. The microscopic structure of the polymer materials, in turn, greatly influences the materials' mechanical properties. Additionally, loading of nanoparticles inside the polymer (e.g., quantum dots for sensing) would influence the mechanical properties of the hybrid materials. The extent to which swollen/shrunken states influence the mechanical properties of the hydrogels is therefore crucial for their final applications [26]. The interactions between the polymer- coated nanoparticles and biological tissue or cells are complex and topography and mechanical properties of the nanoparticles, their chemistry, and behavior in various biological buffers all play a role. The knowledge of the nanomechanical properties of such materials is therefore of fundamental interest for the understanding of how mechanical properties affect the interaction of these materials with their environments, for example, with cells. This 
necessitates the study of changes in the nanoscale mechanical properties of the polymers at interfaces in response to environmental stimuli.

Atomic force microscopy (AFM) has emerged as a unique, versatile tool for the determination of the elastic properties of polymer surfaces on the nanoscale [26-28]. For instance, AFM in force-spectroscopy mode has been used to study the mechanical properties of thin polymer layers [29], polyelectrolyte multilayers [30], and the viscoelastic and plastic properties of amorphous polymer surfaces as a function of temperature [31]. Due to the small dimensions of the AFM probe, related nanoindentation experiments have provided information on the nanomechanical properties, for example, the Young's modulus, of these surfaces with unprecedented lateral resolution [27-29, 32-34]. Recently, AFM has also been used as an efficient tool for the determination of mechanical properties of polymeric systems as a function of environmental conditions such as temperature, [35] $\mathrm{pH}$, [36] or surface charge [37]. Although the structural, as well as thermodynamic and kinetic aspects of the VPT of PNIPAM have been studied in great detail, [15, 38, 39] the nanoscale mechanical properties of PNIPAM microgels across the LCST have not yet been investigated in depth [26, 40-42]. Two studies on the nanomechanical properties of microgels with simultaneous observations of the VPTT behavior by AFM were only recently reported in the literature [42, 43].

Hence, in this chapter, we report on the simultaneous structural and surface nanomechanical properties of individual PNIPAM microgels. PNIPAM microgels are synthesized by surfactant free emulsion radical polymerization. Changes in the particle characteristic dimensions and changes of the surface elastic modulus above and below the VPTT are monitored by in situ Atomic Force Microscopy as a function of imaging conditions. It is found that depending on the imaging conditions, (in air and in water at different temperatures) the PNIPAM surface modulus values span two orders of magnitude form 1.8 $\mathrm{MPa}$ to $125.9 \mathrm{MPa}$. The changes in the surface modulus values obtained correspond to changes in the surface crosslink densities in the range from $10^{-4}$ and $10^{-2} \mathrm{~mol} \mathrm{~cm}^{-3}$.

\subsection{Experimental section}

Synthesis of PNIPAM particles: The monomer $N$-isopropylacrylamide (NIPAM), crosslinker $N, N^{\prime}$-methylenebisacrylamide (BIS), and polymerization initiator potassium persulfate (KPS) were all purchased from Aldrich (Germany). Before use the NIPAM monomer was recrystallized from hexane (HPLC grade, Biosolve). Milli-Q water (18.2 $\mathrm{m} \Omega$, 
Millipore) was used for the synthesis and purification procedures. PNIPAM particles were synthesized by surfactant free radical polymerization according to the procedure reported by Pelton and Chibante [44]. $14 \mathrm{~g} / \mathrm{L}$ of NIPAM and $1.4 \mathrm{~g} / \mathrm{L}$ of BIS were mixed together in 700 $\mathrm{mL}$ of Milli-Q water by continuous stirring under $\mathrm{N}_{2}$ atmosphere in a three-necked flask equipped with a condenser, and placed in a heating bath. The temperature was raised to $70{ }^{\circ} \mathrm{C}$ and then $20 \mathrm{~mL}$ of $0.83 \mathrm{~g} / \mathrm{L}$ of KPS solution was added to initiate the polymerization reaction. The reaction medium turned turbid after 5 minutes, and the reaction was allowed to proceed for 4 hours at $70{ }^{\circ} \mathrm{C}$. After the reaction was stopped, the solution was cooled down to room temperature. To remove the unreacted monomer and low molecular weight oligomeric impurities, the reaction solution was dialyzed using a cellulose membrane (12-14 kDa, 4 Spectra/Por, USA) for one week against deionized water.

Characterization of PNIPAM particles: The VPTT of the synthesized PNIPAM microgels was determined by temperature-dependent light scattering and UV-Vis absorption measurements. The mean diameter value of the PNIPAM microgels in water solutions at room temperature was obtained using a Malvern Zetasizer 4000 (633 nm, 90 scattering angle). The VPTT of the particles was assessed by measuring the particle diameters at different temperatures from $25{ }^{\circ} \mathrm{C}$ to $40{ }^{\circ} \mathrm{C}\left( \pm 0.5{ }^{\circ} \mathrm{C}\right)$. A Varian Cary $300 \mathrm{UV}$-VIS spectrophotometer (USA) equipped with a temperature controller was used to determine the effect of temperature on the absorbance of PNIPAM particles. The absorbance of the polymer solutions was monitored at $500 \mathrm{~nm}$ and recorded for temperatures from $25^{\circ} \mathrm{C}$ to $40^{\circ} \mathrm{C}( \pm 0.2$ $\left.{ }^{\circ} \mathrm{C}\right)$. The diameter of the air-dried microgels was obtained by Scanning Electron Microscopy (SEM) (Jeol JSM-5600 LV, Japan) operated at $5 \mathrm{kV}$. The microgel particles were deposited onto cleaned silicon substrates prior to measurements. The substrates were cleaned using a solution of $30 \%(\mathrm{v} / \mathrm{v})$ hydrogen peroxide and $70 \%(\mathrm{v} / \mathrm{v})$ concentrated sulfuric acid and subsequently stored in ethanol.

Atomic force microscopy: All AFM experiments were performed with a Multimode NanoScope III (Veeco-Digital Instruments, CA, USA). The instrument was equipped with a J-scanner $\left(100 \times 100 \mu^{2} \mathrm{x}-\mathrm{y}\right.$ scan size) and with a high-temperature accessory, diTAC, provided by Veeco (USA). Commercially available triangular silicon nitride cantilevers were used (Veeco, USA).

Concentrated or diluted PNIPAM solutions were deposited directly onto $1 \times 1 \mathrm{~cm}^{2}$ pieces of a silicon wafer and dried in air. The particles were first visualized in air, which was followed by observations in the liquid cell in aqueous solutions. The sample topography in 
air, and water, was obtained by measurements in the contact mode. Temperature dependent experiments in the liquid cell were performed at $25^{\circ} \mathrm{C}$ and $40{ }^{\circ} \mathrm{C}$. To perform simultaneous topographical and force-spectroscopy measurements the AFM was operated in the ForceVolume (FV) mode. The sample height images (512 samples per line) and force-distance curves (64 samples per line, with 64 points per force-distance curve) were acquired at a frequency of $9.3006 \mathrm{~Hz}$. The spring constant of the cantilevers used was calculated according to the procedure reported in [45], where a reference cantilever with a known spring constant $\left(k_{r e f}\right)$ is used. The sensitivity of the cantilever deflection detection system was measured on a hard substrate $\left(\delta_{t o t}\right)$ and at the free end of a reference cantilever $\left(\delta_{\text {test }}\right)$. The following formula (Eq. 4.1) was used to calculate the spring constant of the cantilever $\left(k_{\text {test }}\right)$ :

$$
k_{\text {test }}=k_{\text {ref }} \frac{\delta_{\text {tot }}-\delta_{\text {test }}}{\delta_{\text {test }} \cos \theta}
$$

where $\theta$ is the angle formed between the cantilever under test and the reference cantilever. The calculated cantilever spring constant was equal to $0.46 \pm 0.05 \mathrm{~N} / \mathrm{m}$.

For the evaluation of the surface nanomechanical properties of PNIPAM only the force distance curves on top of each of the spheres were taken for subsequent analysis. To estimate the Young's modulus, the raw deflection-piezo travel data were transformed into force-indentation curves. The sensitivity of the deflection measurements was determined form the slope of the contact part of the deflection-piezo travel curve obtained on a hard silicon substrate. The force $(F)$ was obtained from the deflection data by applying Hooke's law $F=k_{\text {test }} d$, where $\mathrm{d}$ is the cantilever deflection. For each point on the curve, the indentation values were calculated by subtracting the obtained deflection from the piezo displacement.

\subsection{Results and discussion}

The crosslinked PNIPAM microgels were synthesized using surfactant-free radical polymerization. The synthesis was carried out at temperatures above the LCST of PNIPAM in the presence of a crosslinker, the result of which was that as the polymerization proceeded, PNIPAM phase separated and formed a crosslinked network. The stability of the resulting PNIPAM colloidal system is a result of a combination of steric and electrostatic repulsion effects [44]. 
The diameter of representative crosslinked microgel particles as a function of temperature is presented in Figure 4.1. At room temperature (RT), the particles have an average diameter of $550 \mathrm{~nm}$ with a polydispersity index of $0.12 \pm 0.08$ as determined by dynamic light scattering. When the temperature was increased, the diameter decreased slightly; a drop of the particle diameter was observed and then finally the diameter reached a value of approximately $280 \mathrm{~nm}$.

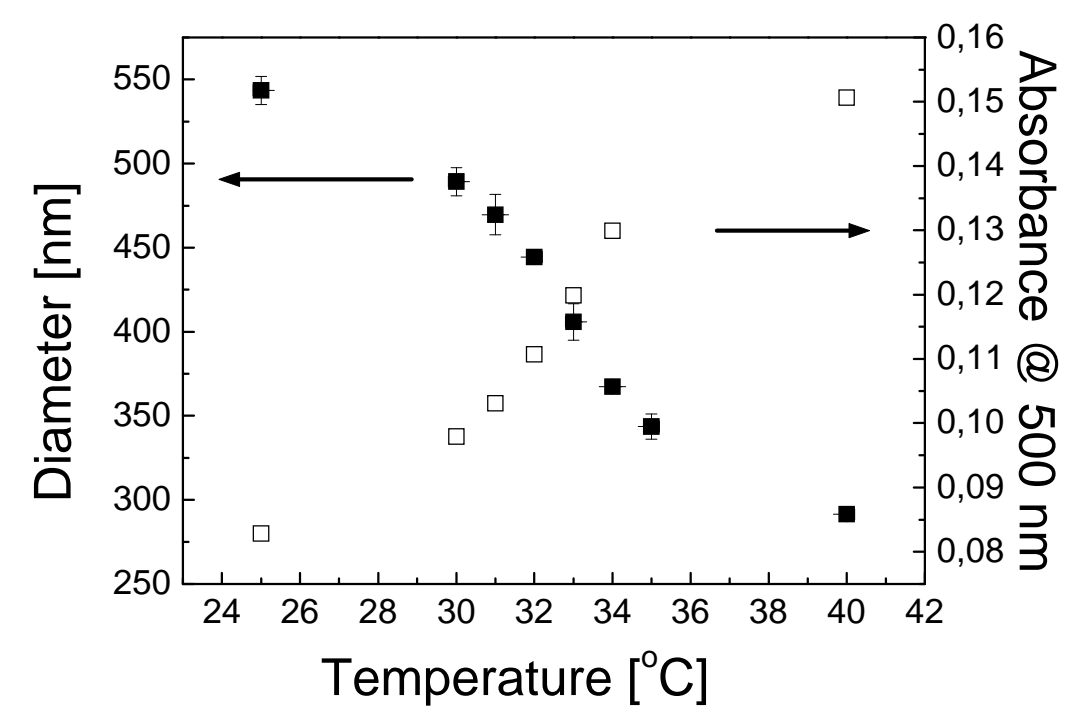

Figure 4.1 . PNIPAM particles diameter ( $\square$ ) and absorbance ( $\square$ ) as a function of temperature. The PNIPAM particle diameter decreases by almost $50 \%$ when crossing the polymer VPTT.

This decrease in particle diameter corresponds to a $\sim 7.6$-fold decrease in particle volume. Such a behavior is a signature of a VPTT, and the temperature, at which the transition occurs, is associated with the LCST of the polymer. The mechanism of this transition is related to the decreasing solvency of PNIPAM in water with increasing temperature $[15,46,47]$.

Using Figure 4.1 and by plotting the first derivative of the particle diameter versus temperature, we estimate the VPTT of the sample to be around $32{ }^{\circ} \mathrm{C}$. This value is in good agreement with the data reported in the literature for the same system [8]. The VPTT can be also monitored by UV-Vis spectroscopy. Temperature-dependent UV-Vis measurements were performed in order to monitor the temperature dependence of the absorbance of the polymer solution at $500 \mathrm{~nm}[48,49]$. The increase of the optical density (OD) of the PNIPAM solution at the monitoring wavelength as a function of temperature is shown in Figure 4.1. It is observed that the OD doubles at T>LCST, when compared to the value 
measured at RT. Such increase in OD implies that thermo-responsive PNIPAM particles collapse at T $>$ LCST and aggregate.

In Figure 4.2a and 2c scanning electron microscopy (SEM) and AFM height images of dry PNIPAM particles obtained at $22{ }^{\circ} \mathrm{C}$ deposited from low-concentration solutions onto the native oxide layer of a silicon wafer are shown, respectively.

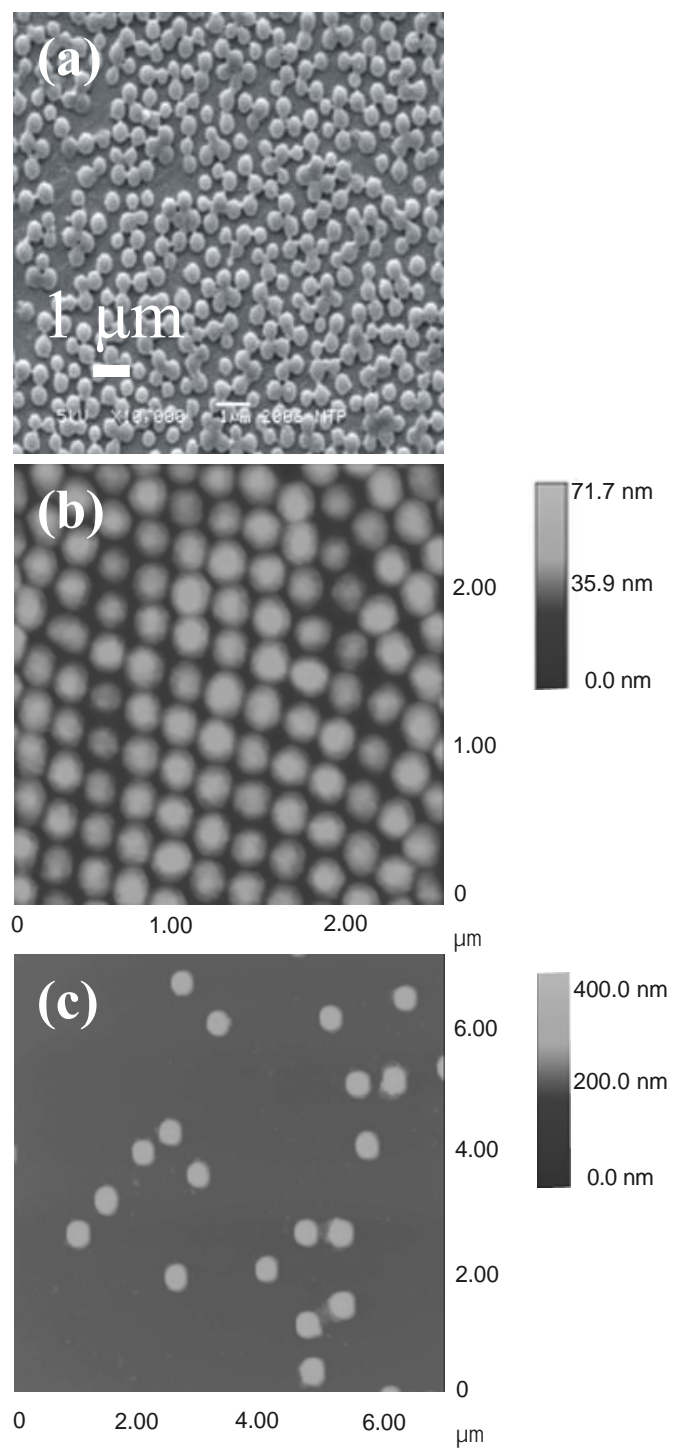

Figure 4.2. (a) SEM image of PNIPAM particles on silicon oxide substrate. AFM height images of PNIPAM deposited from high (b) and low concentration solutions (c). The particles are spherical with a narrow size distribution as evidenced by particle packing exhibiting long-range order in part (b).

When deposited from concentrated solutions (Figure 4.2b), the PNIPAM particles formed a closely packed structure. This structure is likely formed as a result of packing 
constraints and particle interaction. Additionally, the negative charges present at the surface of the PNIPAM particles can stabilize the packing [44].

Height images and cross sections of PNIPAM particles deposited onto silicon substrates, in water, at temperatures below and above the VPTT are shown in Figure 4.3a, and Figure 4.3b, respectively.
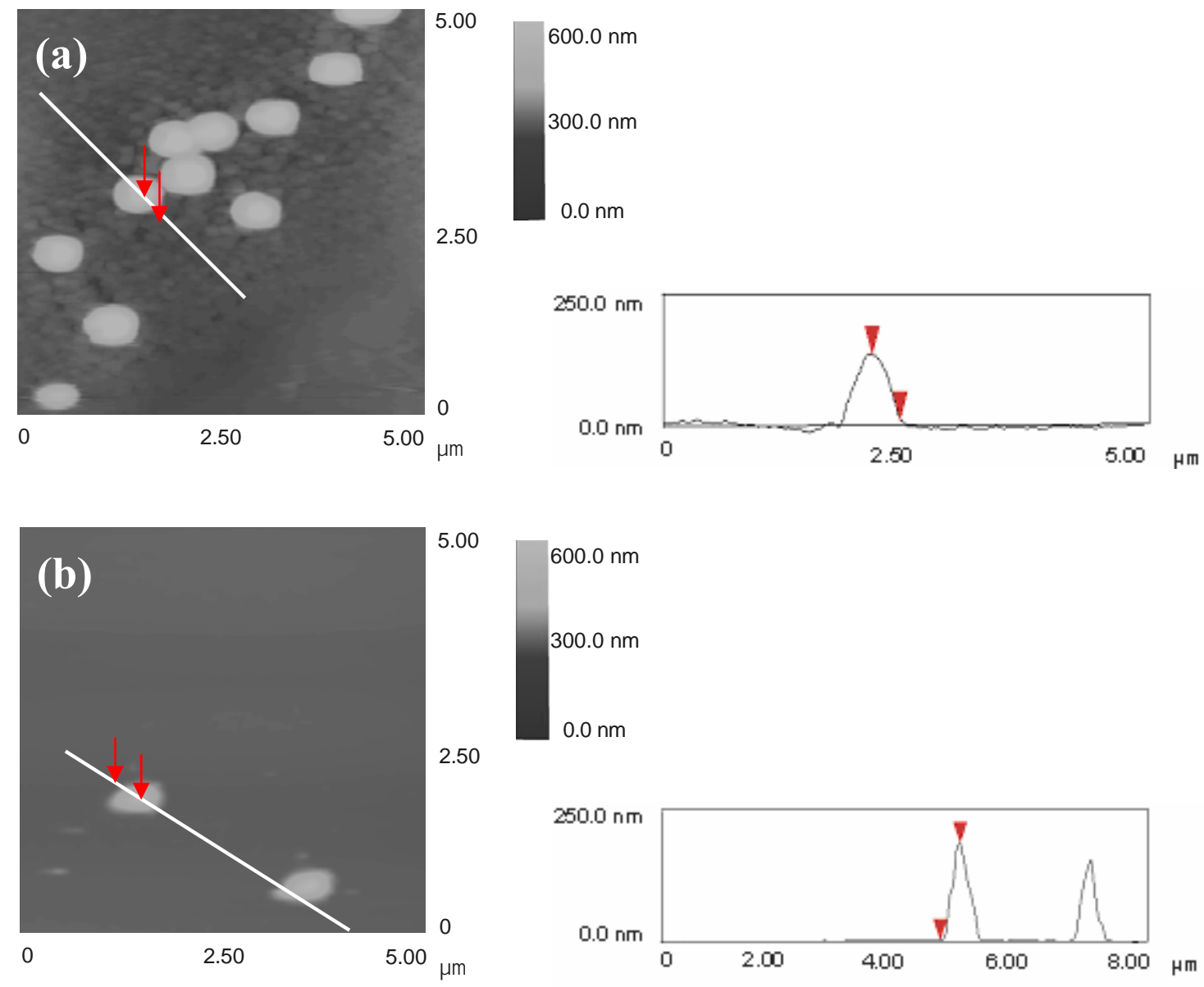

Figure 4.3. AFM height images and corresponding cross sections for PNIPAM particles in water at (a) $22{ }^{\circ} \mathrm{C}$ and at (b) $40{ }^{\circ} \mathrm{C}$.

The cross section analysis was performed on isolated particles and height and width values were extracted. The vertical and horizontal distances between the arrows were taken as apparent height and half-width of the particles. More than 100 isolated particles were analyzed in order to calculate statistically relevant average height and width values for each experimental condition. The results obtained are shown in Table 4.1. The corresponding histograms are displayed in Figure 4.4. 
Table 4.1. Average height and apparent width values for PNIPAM nanoparticles obtained by AFM imaging in air at $22{ }^{\circ} \mathrm{C}$, in water at $22{ }^{\circ} \mathrm{C}$, and in water at $40{ }^{\circ} \mathrm{C}$.

\begin{tabular}{|c|c|c|}
\hline Experimental Conditions & Height [nm] & Width [nm] \\
\hline Air, 22 ${ }^{\circ} \mathbf{C}$ & $142 \pm 18$ & $581 \pm 59$ \\
\hline $\mathbf{H}_{2} \mathbf{O}, \mathbf{2 2} \mathbf{C}$ & $169 \pm 25$ & $405 \pm 76$ \\
\hline $\mathbf{H}_{2} \mathbf{O}, \mathbf{4 0} \mathbf{C}$ & $203 \pm 31$ & $390 \pm 55$ \\
\hline
\end{tabular}

AFM imaging of the PNIPAM particles at $22{ }^{\circ} \mathrm{C}$ in air revealed that the dried particles exhibited a flattened shape with average particle height values being almost four times smaller than their (apparent) widths. Upon introduction of water the particles swelled and an increase in height and a decrease in width were observed (at $22{ }^{\circ} \mathrm{C}$ ). Upon increasing the temperature above the LCST $\left(40^{\circ} \mathrm{C}\right)$, the width of the particles further decreased. However, the height of the particles increased as well. A scheme representing the morphology of particles under three imaging conditions (air at $22{ }^{\circ} \mathrm{C}$, water $22{ }^{\circ} \mathrm{C}$, and water at $40{ }^{\circ} \mathrm{C}$ ) is shown in Figure 4.4c.

In air, at $22^{\circ} \mathrm{C}$, the dried PNIPAM particles adsorbed on the silicon surface adopt a "pancake"-like shape (case I in Figure 4.4c) indicating that the polymer chain network is mildly crosslinked and relatively porous. The increase in height accompanied by a decrease in width for the particles in water indicates that the particles adopt a more rounded shape (case II in Figure 4.4c). At temperature lower than the VPTT the polymer is hydrophilic and water is able to penetrate into the interior of the PNIPAM spheres. As the temperature is increased above the VPTT, a further increase in height was observed with a decrease in width (case III in Figure 4.4c). 

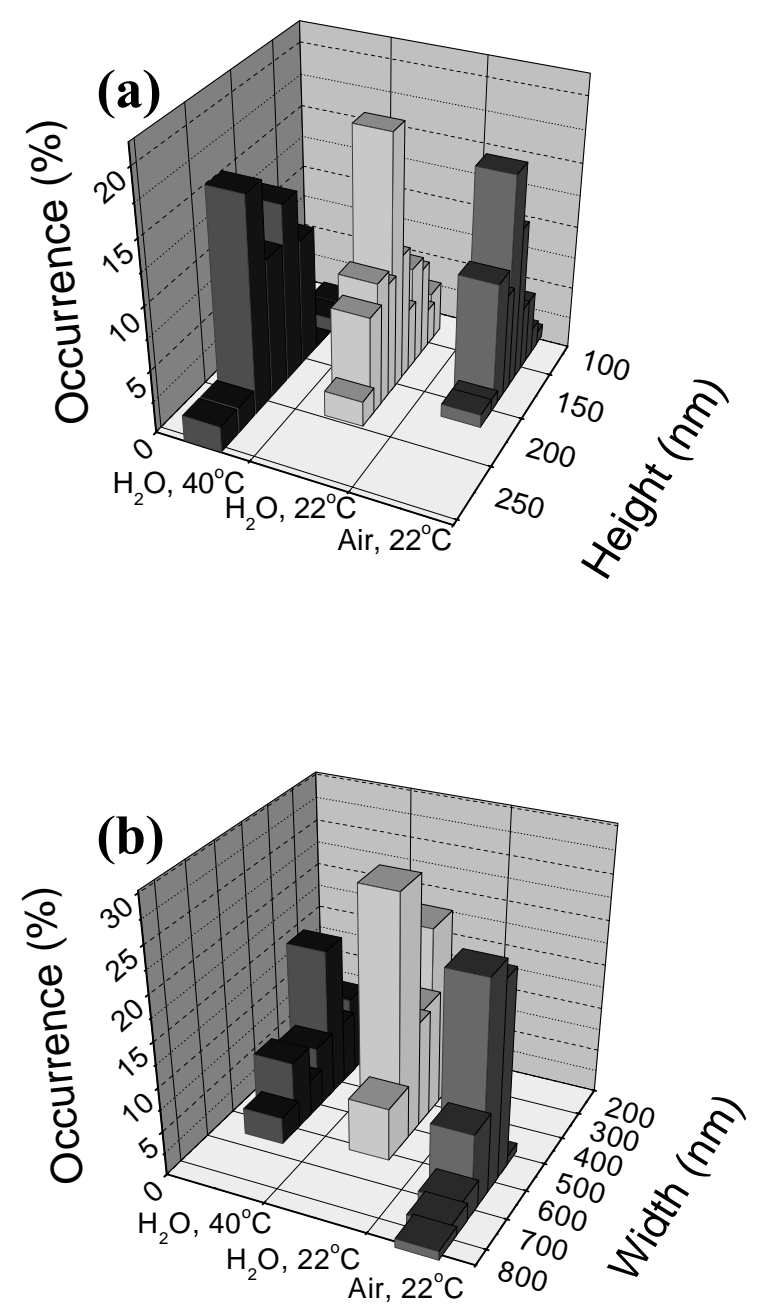

\section{$\begin{array}{lll}\text { I. Air, } 22^{\circ} \mathrm{C} & \text { II. Water, } 22^{\circ} \mathrm{C} & \text { III. Water, } 40^{\circ} \mathrm{C}\end{array}$}

(c)
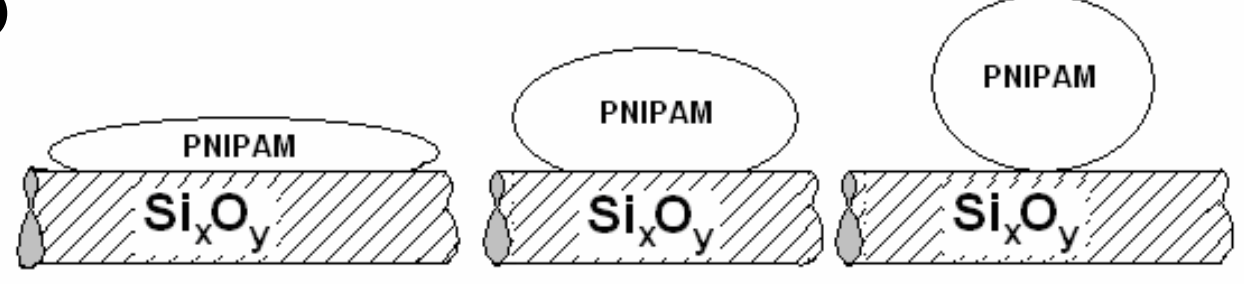

Figure 4.4. (a) Height and, (b) width histograms of PNIPAM particles under different experimental condition, as obtained from the AFM height images. (c) Proposed morphologies of the PNIPAM particles at different experimental conditions. In air, at $22{ }^{\circ} \mathrm{C}$, (I) the particle are dry and collapsed (T<VPTT). In water, at $22{ }^{\circ} \mathrm{C}$, (II) the particles swell (T<VPTT), and finally in water, at $40{ }^{\circ} \mathrm{C}$ (T>VPTT) (III), the hydrophobic particles tend to decrease the contact area with the hydrophilic substrate. 
When crossing the LCST of PNIPAM the polymer becomes hydrophobic and the water should be expelled from the particle interior, resulting in a decrease of the average particle size. The increase in height observed in our study seems to contradict this statement. However, it should be kept in mind that the PNIPAM particles are adsorbed onto a hydrophilic silicon oxide substrate. It is reasonable to argue that morphological changes of surface-adsorbed PNIPAM will be controlled not only by the interactions between the polymer chains with each other, and with the surrounding medium, but also with the substrate. The native oxide surface of the silicon substrates becomes hydrophilic after treatment with a Piranha solution. At T $>$ VPTT the PNIPAM particles become hydrophobic and tend to decrease the contact area with the hydrophilic substrate. This in turn will result in an increase in height. Interactions between the PNIPAM chains and the substrate at T $<$ VPTT would be also responsible for the nonspherical, globular, morphology of the PNIPAM particles. To substantiate this claim, we imaged PNIPAM particles deposited on a hydrophobic graphite substrate above the LCST in water. The measured average height and apparent width values were found to be $142 \pm 30 \mathrm{~nm}$ and $300 \pm 48 \mathrm{~nm}$, respectively. Clearly, in the case of a hydrophobic substrate the particles became flattened normal to the substrate for T $>$ LCST. The wetting properties of the substrate therefore play a major role in defining the final particle morphology.

The particle volume of the microgel was estimated from AFM height and width data assuming that the particles have spheroid morphology on the surface. For PNIPAM on silicon substrate the particle volume at $\mathrm{T}<\mathrm{LCST}$ was approximately doubled with respect to the size observed at T>LCST. This increase is significantly lower than the eight fold change in volume obtained from light scattering experiments. However, due to tip convolution effects, the real width, thus the volume, can not be accurately determined.

AFM force-volume (FV) imaging was used for the simultaneous determination of topography and indentation modulus of the PNIPAM particles. FV imaging is an AFM surface-mapping technique, in which a force-distance curve is obtained at each pixel of the image. The force-distance curves can be used to determine the value of the indentation modulus. It is therefore possible to correlate topographical features with the adhesive and mechanical properties. An example of such force-volume images obtained for PNIPAM deposited on silicon in air, at $22{ }^{\circ} \mathrm{C}$, is shown in Figure 4.5. A clear correlation between the topography (Figure 4.5a) and the force-volume image (Figure 4.5b) is visible. This allows us to preferentially choose the force-distance curves obtained only on top of the PNIPAM spheres, i.e. to probe indentation modulus in the surface-normal direction. Such procedure 
also ensures that e.g. no probing at the nanoparticle side-walls was performed (increased contact areas can influence the measured values).
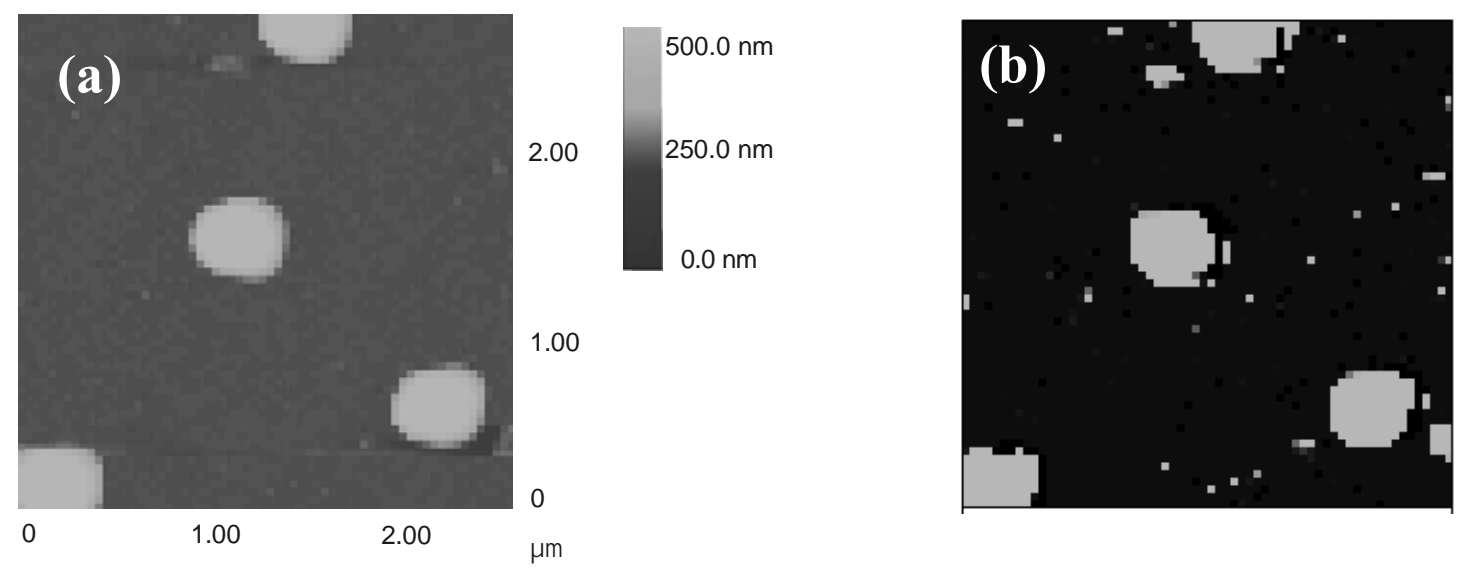

Figure 4.5. Representative height (a) and force volume (b) images obtained simultaneously for isolated PNIPAM spheres in air at $22^{\circ} \mathrm{C}$. Correlation between the height and force-volume is clearly visible. The force distance curves are collected on top of each of the spheres. High adhesion corresponds to light color in the force volume image.

In order to quantitatively compare the surface mechanical properties we proceed with the calculation of the surface elastic modulus. In order to calculate the elastic modulus of the samples, the cantilever deflection $(d)$ and piezo displacement $(z)$ data were converted into force and indentation, respectively. The analysis method employed in this study assumes that the material recovery during the initial unloading is largely elastic. Another assumption is that during the initial withdrawal of the AFM tip indenter, the contact area between the indenter and the sample remains constant $[50,51]$. To further ensure that the deformations probed are largely elastic only the initial slope of the retraction part of the force-indentation curve was used for the calculations. The elastic modulus was calculated according to the method reported by Oliver and Pharr as shown in Eq. 4.2 [50, 51]:

$$
E=\left(1-v^{2}\right) \frac{\sqrt{\pi}}{2} \frac{d P}{d h} \frac{1}{\sqrt{A}}
$$

where $v$ is the Poisson ratio, $d P / d h$ is the slope of the retraction curve and $A$ is the contact area between the tip and the sample. The contact area was calculated using the Eq. 4.3: 


$$
A=3 \sqrt{3} h_{p}^{2} \tan ^{2} \alpha
$$

where $\alpha$ is the half angle of the indenter and $h_{p}$ is the effective penetration depth [51] determined from the force-indentation curve.

A set of force-indentation curves for PNIPAM particles and the native oxide surface of the silicon substrate under each experimental condition are shown in Figure 4.6.

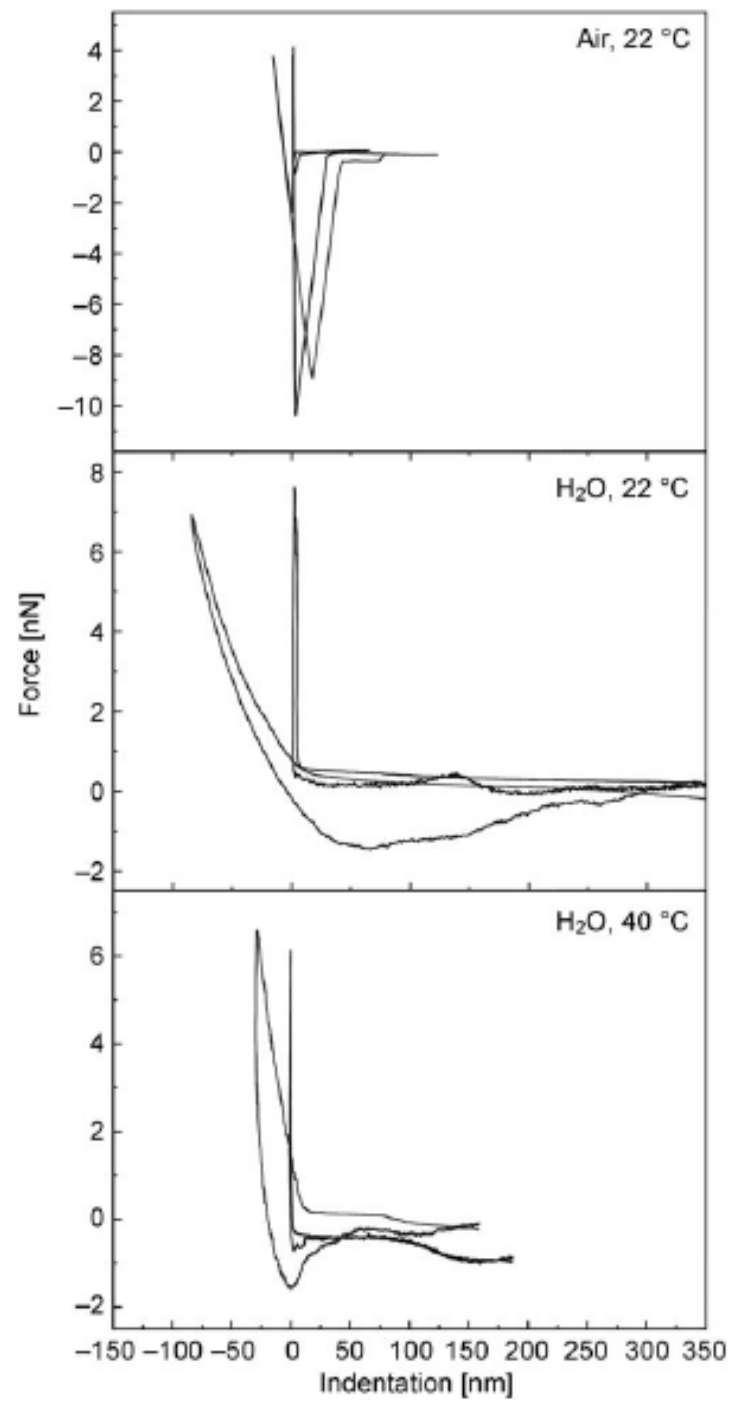

Figure 4.6. Representative force-indentation curves obtained from force-distance curves collected on top of the spheres for isolated PNIPAM spheres in air at $22^{\circ} \mathrm{C}$, in water at $22^{\circ} \mathrm{C}$ and in water at $40^{\circ} \mathrm{C}$. The penetration depth is the highest for the swollen particles measured in water at $22^{\circ} \mathrm{C}$. The indentation is the smallest for the air-dried samples measured at $22^{\circ} \mathrm{C}$. Two sets of curves are shown for each experimental condition. Those curves with a sharp increase in force correspond to curves acquired on hard substrate, while the remaining ones were obtained on the microgels. 
The 'zero indentation' for hard silicon surface was taken as the reference for determination of penetration depths for PNIPAM particles. The highest penetration was observed in the measurements performed at $22^{\circ} \mathrm{C}$ in water. Crossing the VPTT, the penetration depth decreased by approximately $50 \%$, indicating presence of a stiffer surface. The initial slopes of the retraction curves were used to calculate the modulus values. It should be noted at this stage that because the maximum indentation depths are in the range of tens of nanometers, the assumption of pyramidal tip geometry (Eq. 4.3) can introduce errors into calculations and the calculated modulus values could be slightly overestimated. In addition, we applied the approach reported by Akhremitchev et al. [52] to estimate the correction factors related to the substrate effects on the modulus values.

The value of the elastic modulus obtained for dry PNIPAM spheres in air was estimated to be $125.9 \pm 0.9 \mathrm{MPa}$. When the spheres swell in water below the VPTT their modulus dramatically decreases by almost two orders of magnitude to $1.8 \pm 0.2 \mathrm{MPa}$. Above the VPTT the elastic modulus of the spheres increases an order of magnitude to $12.8 \pm 3.6$ $\mathrm{MPa}$. In other words, upon shrinking the cross-linked particles lose water and become stiffer. This trend in the elastic modulus of PNIPAM particles at temperatures above the LCST is in good agreement with the values reported e.g. by Wiedemair et al. [42], where a 15 fold decrease in elasticity was observed above the LCST of PNIPAM.

Rubber elasticity behavior of crosslinked systems is governed by the effective crosslink density of the elastically active junctions. It was previously reported that in the case of 'ideal' vulcanized rubber containing no network defects in the form of 'loose ends', or, intramolecular crosslinks, the resulting network elasticity can be described by the Eq. 4.4 [53]:

$$
G=\frac{E}{3}=\frac{\rho R T}{M_{c}}
$$

where $G$ is the shear modulus $(\mathrm{Pa}), E$ is the Young's modulus of elasticity (Pa), $\rho / M_{c}$ is the density of the elastically active crosslinks $\left(\mathrm{mol} \mathrm{cm}^{-3}\right)$ and $R$, and $T$ are the universal gas constant and temperature, respectively. Here, we correlate the elasticity of PNIPAM particles measured under three different conditions (air at $22^{\circ} \mathrm{C}, \mathrm{H}_{2} \mathrm{O}$ at $22^{\circ} \mathrm{C}$, and $\mathrm{H}_{2} \mathrm{O}$ at $40^{\circ} \mathrm{C}$ ) to the crosslink densities per probed volume by using the Eq. 4.4. The crosslink density of the elastically active network junctions (physical as well as chemical crosslinks) determined for our air-dried particles at $22^{\circ} \mathrm{C}$ is $\left.1.71( \pm 0.1) \times 10^{-2}(\mathrm{~mol} \mathrm{~cm})^{-3}\right)$ and this value decreases dramatically to $2.44( \pm 0.27) \times 10^{-4}\left(\mathrm{~mol} \mathrm{~cm}^{-3}\right)$ upon water uptake by the particles. At 
temperatures higher than LCST of the PNIPAM, the crosslink density increases almost ten fold to $1.64( \pm 0.46) \times 10^{-3}\left(\mathrm{~mol} \mathrm{~cm}^{-3}\right)$.

For PNIPAM microgels in water an almost 10 -fold increase in crosslink density is observed for a twofold decrease in the particle volume. Considering the correlation reported in Reference [52] for natural rubber, we would anticipate only a twofold increase in crosslink density for a twofold decrease in the particle volume. It follows that the AFM indentation experiments probe not only the chemical crosslinks but also the formation of new physical crosslinks at temperatures higher than LCST [15].

The synthesis of PNIPAM microgels takes place at temperatures higher than LCST, where the individual PNIPAM chains are in the collapsed state. As the temperature is lowered to $22^{\circ} \mathrm{C}$, the particles swell in water. During swelling the physical crosslinks are inevitably broken. Upon increasing the temperature above the LCST, the chains collapse and the effective increase in the number of crosslinks is attributed to physical crosslinks. These crosslinks can be associated with the hydrophobic interactions between isopropyl groups.

It has been shown that PNIPAM microgel particles (obtained under very similar conditions) exhibit heterogeneous core/shell structure with a highly crosslinked core and a nearly noncrosslinked shell [54]. Hence, we may assume that the crosslinks sensed by AFM, which are near the corona, are mostly physical. Thus, the observed change in crosslink density can be primarily attributed to the changes in the number of physical crosslinks upon passing the LCST.

Since the formation of physical crosslinks requires intermolecular interactions between polymer chains, it is important to study how the amount of the crosslinker introduced during the synthesis procedure would influence the formation of the physical crosslinks. In particular, functionalized nanoparticles would act as multiple crosslink points and influence the nanomechanical behavior of the PNIPAM hydrogels by preventing intermolecular interactions [25].

\subsection{Conclusions}

AFM was used to probe the morphology and the nanomechanical properties of individual PNIPAM microgels deposited on surfaces of silicon oxide. The particles were imaged in air and in water at temperatures below $\left(22^{\circ} \mathrm{C}\right)$ and above $\left(40^{\circ} \mathrm{C}\right)$ the polymer VPTT $\left(32^{\circ} \mathrm{C}\right)$. The measured height and apparent width values of the PNIPAM particles in water revealed that the particle volume changed by a factor of approximately two upon crossing the VPTT. The particle morphology also changed, surprisingly, from a flat 
pancake-like to a more spherical, round shape upon increasing the temperature. Decreased solubility of the particles at T>VPTT, as well as the changes in the interactions between the particles with the substrate were mainly responsible for the observed morphological changes. Modulus values and corresponding effective crosslink densities were quantitatively determined by AFM nanoindentaion experiments. The change of the surface modulus in water when crossing the VPTT was approximately ten fold. We attribute the changes in the crosslink densities to a combination of the chemical crosslinks and introduction of large number of physical crosslinks above the VPTT of PNIPAM microgels.

\subsection{References}

1- Winnik, F.M.; Whitten, D.G., Editorial Langmuir 2007, 23: p. 1.

2- $\quad$ Li, Y.; Tanaka, T. J. Chem. Phys. 1990, 92: p. 1365.

3- Duracher, D.; Sauzedde, F.; Elaissari, A.; Perrin, A.; Pichot, C., Colloid Polym. Sci. 1998, 276: p. 219.

4- Moselhy, J.; Wu, X.Y.; Nicholov, R.; Kodaria, K., J. Biomater. Sci. Polym. Ed. 2000, 11: p. 123.

5- $\quad$ Sershen, S.R.; Westcott, S.L.; Halas, N.J.; West, J.L., J. Biomed. Mater. Res. 2000, 51: p. 293.

6- $\quad$ Tanaka, T.; Nishio, I.; Sun, S.T.; Ueno-Nishio, S., Science 1982, 218: p. 467.

7- $\quad$ Osada, Y.; Gong, J.P., Adv. Mater. 1998, 10: p. 827.

8- $\quad$ Heskins, M.; Guillet, J.E., J. Macromol. Sci. Chem 1968, A2: p. 1441.

9- $\quad$ Kasgoz, H.; Ozgumus, S.; Orbay, M., Polymer 2004, 44: p. 1785.

10- Peppas, N.A.; Hilt, J.Z.; Khademhosseini, A.; Langer, R., Adv. Mater, 2006 18: p. 1345.

11- Lee, K.Y.; Mooney, D.J., Chem.Rev. 2001, 101: p. 1869.

12- Lopez, C.V.; Raghavan, S. L.; Snowden, M. J., React. Funct. Polym. 2004, 58: p. 175.

13- Gerlach, G.; Günther, M.; Suchaneck, G.; Sorber, J.; Arndt, K.-F.; Richter, A., Macromol. Symp. 2004, 21: p. 403.

14- Cornelius, V. J.; Snowden, M. J.; Silver, J.; Fern, G. R., React. Funct. Polym. 2004, 58: p. 165.

15- Schild, H.G., Prog. Polym. Sci. 1992, 17: p. 163.

16- Pelton, R.H., Macromol Symp. 2004, 207: p. 57.

17- Lu, Y.; Mei, Y.; Drechsler, M.; Ballauff, M., Angew.Chem. 2006, 118: p. 827; Angew. Chem. Int. Ed. 2006, 45: p. 813. 
18- Suzuki, D.; Kawaguchi, H., Langmuir 2006, 22: p. 3818.

19- Pardo-Yissar, V.; Gabai, R.; Shipway, A.N.; Bourenko, T.; Willner, I., Adv. Mater. 2001, 13: p. 1320.

20- Wang, C.; Flynn, N.T., Langer, R., Adv. Mater. 2004, 16: p. 1074.

21- Kim, S.-W.; Kim, S.; Tracy, J.B.; Jasanoff, A.; Bawendi, M.G., J. Am. Chem. Soc. 2005, 127: p. 4556.

22- Zhao, X.; Ding, X.; Deng, Z.; Zheng, Z.; Peng, Y.; Long, X., Macromol. Rapid Comm. 2005, 26: p. 1784.

23- Gatas-Asfura, K.M.; Zheng, Y.; Micic, M.; Snedaker, M.J.; Ji, X.; Sui, G.; Orbulescu, J.; Andreopoulos, F.M.; Pham, S.M.; Wang, C.; Leblanc, R.M., J. Phys. Chem. B 2003, 107: p. 10464.

24- Kuang, M.; Wang, D.; Bao, H.; Gao, M.; Möhwald, H.; Jiang, M., Adv. Mater. 2005, 17: p. 267.

25- Gong, Y.; Gao, M.; Wang, D.; Möhwald, H., Chem. Mater. 2005, 17: p. 2648.

26- Matzelle, T.R.; Geuskens, G.; Kruse, N., Macromolecules 2003, 36: p. 2926.

27- Briscoe, B.; Fiori, L.; Pelillo, E., J. Phys. D 1998, 31: p. 2395.

28- Butt, H.-J. ; Cappella, B. ; Kappl, M., Surf. Sci. Rep. 2005, 59: p. 1.

29- Dimitriadis, E.K.; Horkay, F.; Maresca, J.; Kachar, B.; Chadwick, R.S., Biophys. J. 2002, 82: p. 2798.

30- Mermut, O.; Lefebre, J.; Gray, D.G.; Barrett, C.J., Macromolecules 2003, 36: p. 8819.

31- Cappella, B.; Kaliappan, S.K.; Sturm, H., Macromolecules 2005, 38: p. 1874.

32- Chizhik, S.A.; Huang, Z.; Gorbunov, V.V.; Myshkin, N.K.; Tsukruk, V.V., Langmuir 1998, 14: p. 2606.

33- Reynaud, C.; Sommer, F.; Quet, C.; El Bounia, M.; Duc, T.M., Surf. Interface Anal. 2000, 30: p. 185.

34- Raghavan, D.; Gu, X.; Nguyen, T.; VanLandingham, M.R.; Karim, A., Macromolecules 2000, 33: p. 2573.

35- Kaliappan, S.K. and Cappella, B., Polymer 2005, 46: p. 11416.

36- Yao, K.D.; Liu, W.G.; Lin, Z.; Qiu, X.H., Polym. Int. 1999, 48: p. 794.

37- Tan, S.; Sherman Jr., R.L.; Ford, W.T., Langmuir 2004, 20: p. 7015.

38- Rice, C.V., Biomacromolecules 2006, 7: p. 2923.

39- Tanaka, T.; Sato, E.; Hirokawa, Y.; Hirotsu, S.; Peetermans, J., Phys. Rev. Lett. 1985, 55: p. 2455. 
40- Suzuki, A.; Yamazaki, M.; Kobiki, Y.; Suzuki, H., Macromolecules 1997, 30: p. 2350.

41- Harmon, M.E., Langmuir 2003, 19: p. 10660.

42- Wiedemair, J.; Serpe, M.J.; Kim, J.; Masson, J-F.; Lyon, L.A.; Mizaikoff, B.; Kranz, C., Langmuir 2007, 23: p. 130.

43- Hofl, S.; Zitzler, L.; Hellweg, H.; Herminghaus, S.; Mugele, F., Polymer 2007, 48: p. 245.

44- Pelton, R.H.; Chibante, P., Colloids \& Surfaces 1986, 20: p. 247.

45- $\quad$ Tortonese, M.; Kirk, M., Proc. SPIE 1997, 3009: p. 53.

46- Pelton, R.H., Adv. Coll. Int. Sci. 2000, 85: p.1.

47- Nayak, S.; Lyon, L.A., Angew.Chem. 2005, 117: p. 7862; Angew. Chem. Int. Ed. 2005, 44: p. 7686.

48- Maeda, T.; Kanda, T.; Yonekura, Y.; Yamamoto, K.; Aoyagi, T., Biomacromolecules 2006, 7: p.545.

49- Mori, T.; Maeda, M., Langmuir 2004, 20: p. 313.

50- $\quad$ Pharr, G.M.; Oliver, W.C.; Botzen, F.R., J. Mater. Res. 1992, 7: p. 613.

51- Oliver, W.C.; Pharr, G.M., J. Mater. Res. 1992, 7: 1564.

52- Guo, S.; Akhremitchev, B.B., Biomacromolecules 2006, 7: p. 1630.

53- Treloar, L. R. G. The Physics of Rubber Elasticity, 3rd ed.; Oxford Clarendon Press: England, $1975 ; 160$ pp.

54- Fernandez-Barbero, A.; Fernandez-Nieves, A. ; Grillo, I. ; Lopez-Cabaros, E., Phys Rev E 2002, 66 : p. 051803 
40- Suzuki, A.; Yamazaki, M.; Kobiki, Y.; Suzuki, H., Macromolecules 1997, 30: p. 2350.

41- Harmon, M.E., Langmuir 2003, 19: p. 10660.

42- Wiedemair, J.; Serpe, M.J.; Kim, J.; Masson, J-F.; Lyon, L.A.; Mizaikoff, B.; Kranz, C., Langmuir 2007, 23: p. 130.

43- Hofl, S.; Zitzler, L.; Hellweg, H.; Herminghaus, S.; Mugele, F., Polymer 2007, 48: p. 245.

44- Pelton, R.H.; Chibante, P., Colloids \& Surfaces 1986, 20: p. 247.

45- $\quad$ Tortonese, M.; Kirk, M., Proc. SPIE 1997, 3009: p. 53.

46- Pelton, R.H., Adv. Coll. Int. Sci. 2000, 85: p.1.

47- Nayak, S.; Lyon, L.A., Angew.Chem. 2005, 117: p. 7862; Angew. Chem. Int. Ed. 2005, 44: p. 7686.

48- Maeda, T.; Kanda, T.; Yonekura, Y.; Yamamoto, K.; Aoyagi, T., Biomacromolecules 2006, 7: p.545.

49- Mori, T.; Maeda, M., Langmuir 2004, 20: p. 313.

50- $\quad$ Pharr, G.M.; Oliver, W.C.; Botzen, F.R., J. Mater. Res. 1992, 7: p. 613.

51- Oliver, W.C.; Pharr, G.M., J. Mater. Res. 1992, 7: 1564.

52- Guo, S.; Akhremitchev, B.B., Biomacromolecules 2006, 7: p. 1630.

53- Treloar, L. R. G. The Physics of Rubber Elasticity, 3rd ed.; Oxford Clarendon Press: England, $1975 ; 160$ pp.

54- Fernandez-Barbero, A.; Fernandez-Nieves, A. ; Grillo, I. ; Lopez-Cabaros, E., Phys Rev E 2002, 66 : p. 051803 



\section{Chapter 5}

\section{Temperature modulated quenching of quantum dots covalently coupled to chain ends of poly( $\mathrm{N}$-isopropyl acrylamide) brushes on gold*}

A thermo-responsive polymer/quantum dot platform based on poly( $N$-isopropyl acrylamide) (PNIPAM) brushes 'grafted from' a gold substrate and quantum dots (QDs) covalently attached to the PNIPAM layer is presented. The PNIPAM brushes are grafted from the gold surface using an iniferter initiated controlled radical polymerization. The PNIPAM chainends are functionalized with amine groups for coupling to water-dispersible COOHfunctionalized QDs. Upon increasing the temperature above the lower critical solution temperature (LCST) of PNIPAM the QD luminescence is quenched. The luminescence was observed to recover upon decreasing the temperature below the LCTS. The data obtained are consistent with temperature-modulated thickness changes of the PNIPAM layer and quenching of the QDs by the gold surface via nonradiative energy transfer. Such systems may constitute useful combination of QDs and polymers for example in sensing applications.

\footnotetext{
Parts of this chapter have been published in: Tagit, O., Tomczak, N., Benetti, E.M., Cesa, Y., Blum, C., Subramaniam, V., Herek, J.L., Vancso G.J. Temperature-modulated quenching of quantum dots covalently coupled to chain ends of poly(N-isopropyl acrylamide) brushes on gold Nanotechnology 201 - 6 (2009)
} 



\subsection{Introduction}

Optical transduction of stimulus-induced events in polymeric thin film materials is often explored for various sensing applications $[1,2]$. In this context, polymer brushes $[3,4]$ made of polymers undergoing distinct conformational changes [5] in response to changes in $\mathrm{pH}[1][6,7]$, electric field, solvent composition [8], light absorption, temperature [9, 10], or to biochemical stimulus [11] are conveniently used as responsive layers. $\operatorname{Poly}(N-$ isopropylacrylamide) (PNIPAM) is a temperature-responsive polymer that undergoes a reversible phase transition in water when crossing the polymer lower critical solution temperature (LCST, $\sim 32^{\circ} \mathrm{C}$ ) [12]. Above the LCST, the PNIPAM chains are hydrophobic and are in a collapsed state while below the LCST the PNIPAM chains are hydrophilic and extend in water. Optical transduction of this temperature modulated PNIPAM phase transition can be realized by combining nanoparticles with polymer brushes and monitoring their optical properties as a function of temperature. Here, we probe the phase transition of PNIPAM brushes using semiconductor nanocrystals (i.e. quantum dots, QDs).

QDs have gained much attention as optical transducers due to their unique composition and size-tuneable optical properties, broad excitation and narrow emission spectra, and high chemical and photochemical stability [13, 14]. Strategies to immobilize QDs on different types of polymeric surfaces include adhesion [15], adsorption via electrostatic interactions [16], ligand exchange [17] and covalent attachment [7]. Covalent attachment of QDs to polymer layers offers some advantages. Namely, it reduces the possible problems associated with particle aggregation, weak or reversible bonding, and polymer film infiltration [17]. Combining stimulus responsive surfaces and semiconductor nanoparticles allows one to fabricate robust and cost-effective sensors for various applications.

In this contribution, we prepare a hybrid multilayer Au/PNIPAM/QD assembly and probe its optical response to changes of temperature in water. The PNIPAM brushes are grafted from the gold surface using an iniferter initiated controlled radical polymerization. The PNIPAM chain ends are functionalized with amine groups for covalent coupling to carboxylic acid-functionalized QDs. Upon heating and crossing the LCST of PNIPAM, the brushes collapse and QD luminescence intensity decreases. Upon cooling the assembly, the QD luminescence increases again, demonstrating the reversibility of the system. We attribute the decrease and recovery of the luminescence intensity to the non-radiative energy transfer to the gold surface. The data obtained are consistent with temperature modulated thickness changes of the PNIPAM layer and quenching of the QDs by the proximity of the gold surface. 


\subsection{Experimental section}

Core/shell CdSe/ZnS quantum dots were obtained as reported in [18]. The QDs were dispersed in water using mercaptoacetic acid (MAA, 97\%, Aldrich) as reported in [19]. Photopolymerization of NIPAM from the gold substrate was performed using a disulfidebased photo-initiator dithio diundecane-11,1-diylbis $\{4[([($ diethylamino $)$ carbonothioyl $]$ thioethyl)phenyl]carbamate (DTCA) synthesized as reported in [20]. 1,2dioctadecyldisulfane (DDS) was obtained by oxidation of octadecane-1-thiol (ODT, Sigma) in chloroform using an equimolar aqueous solution of iodine and potassium iodide (Merck and Sigma, respectively). To obtain the initiator layer on the gold surface, the gold substrates were incubated overnight in $10 \mathrm{mM}$ chloroform mixed solution of the initiator DTCA and DDS (1:1 molar ratio). The substrates were then placed in a quartz flask equipped with a 280 $\mathrm{nm}$ cut-off optical filter and containing 5\% aqueous solution of NIPAM (Aldrich, recrystallized from hexane before use). The flasks were purged with nitrogen and subsequently irradiated for 10 minutes by UV-B lamps (280-320 nm range, $15 \mathrm{~W}$ G15T8E, Ushio Japan) at a distance of $20 \mathrm{~cm}$. After the photopolymerization, the substrates were extensively rinsed with water and methanol and dried in nitrogen. After grafting the PNIPAM to the gold surface, the substrates were placed in a quartz flask containing $5 \mathrm{~mL}$ of a 0.01M aqueous solution of 4-Amino-2,2,6,6-tetramethylpiperidine-1-oxyl (4-amino-TEMPO) and were irradiated again for 5 minutes by UV-B lamps. The substrates were subsequently rinsed with methanol and dried in nitrogen stream. It was previously shown that the height of PNIPAM brushes obtained with this procedure is around $10 \mathrm{~nm}[20]$.

To attach MAA-functionalized QDs covalently to the PNIPAM chain ends, $76 \mathrm{mg}$ of (1-ethyl-3-[3-dimethylaminopropyl]carbodiimide) (EDC, Aldrich) and $12 \mathrm{mg}$ of $\mathrm{N}$ hydroxysuccinimide (NHS, Aldrich) were separately dissolved in $1 \mathrm{~mL}$ PBS buffer $(0.01 \mathrm{M}$, $\mathrm{pH}$ 7.4) and then mixed in a quartz flask containing gold substrates with PNIPAM brushes. The reaction was left to proceed with gentle shaking for 30 minutes in dark conditions. After the reaction, in order to remove the excess of physisorbed QDs, the substrates were rinsed with methanol and dried in nitrogen.

The UV-Vis absorption spectra of TOPO-coated CdSe/ZnS QDs in chloroform and MAA-coated QDs in water were obtained using a Varian Cary $300 \mathrm{UV}-\mathrm{V}$ is spectrometer. The concentration of the QD solutions and the diameter of the QDs was estimated based on the absorbance of the first absorption peak following the procedure reported in [21]. Luminescence emission spectra were obtained using an Edinburgh FL 900 spectrofluorometer. 
Atomic force microscope (AFM) was used to characterize the morphology of the gold substrates and the PNIPAM polymer brushes before and after QD immobilization. The imaging was performed using a NanoScope III (Veeco-Digital Instruments, CA, USA) AFM equipped with an EV scanner (15 x $15 \mu \mathrm{m}$ scan size). Commercially available triangular silicon nitride cantilevers $(\mathrm{k}=0.3 \mathrm{~N} / \mathrm{m}$, Veeco, CA, USA) were used for all measurements.

Fluorescence images of the QD/PNIPAM brush layers were obtained using a custombuilt microscope based on an inverted Olympus IX71 microscope incorporating spectroscopic capabilities and equipped with suitable filters and dichroic mirrors. Fluorescence images were obtained using a Mercury lamp (Olympus, Japan) as excitation light source, a 40X objective and a sensitive colour camera (AxioCam HRC, Zeiss, Germany). Fluorescence spectra were obtained by $469 \mathrm{~nm}$ laser excitation and confocal detection of the emitted light via a prism spectrometer by a cooled CCD camera (Newton EMCCD, Andor, Northern Ireland). The size of the excitation spot was $\sim 3 \mu \mathrm{m}$. For measurement in aqueous environment, a drop of Milli Q (Millipore, 18.2 M $\Omega$ ) water was placed on top of the sample. A PDMS seal was used to avoid water-spillage. A custom made heating stage was used to perform the above-described photoluminescence imaging at $25{ }^{\circ} \mathrm{C}\left(+/-1{ }^{\circ} \mathrm{C}\right)$ and $50{ }^{\circ} \mathrm{C}\left(+/-2{ }^{\circ} \mathrm{C}\right)$. The imaging experiments were performed 30 minutes after the input of the initial temperature settings ensuring that the temperature drift was minimized.

\subsection{Results and discussion}

$\mathrm{CdSe} / \mathrm{ZnS}$ core-shell quantum dots are commonly synthesized by pyrolysis of organometallic precursors in the presence of organic coordinating solvents [18]. Among the methods used to render the QDs dispersible in water, a ligand exchange reaction with bifunctional ligands is commonly performed [19, 22, 23]. This type of ligand has a functional group which is able to bind to the surface of the QDs and a hydrophilic part (usually $\mathrm{COOH}$ or $\mathrm{OH}$ ) which makes the QDs dispersible in aqueous medium. We have replaced the TOPO ligands on the QD surface with MAA (see experimental section). MAA-coated QDs were dispersible in water with no obvious signs of nanoparticle aggregation. We present the absorption and emission (excited at $460 \mathrm{~nm}$ ) spectra of QDs before and after MAA coating in Figure 5.1., which was chosen among the ligand exchange reactions we performed for QDs of different sizes. 


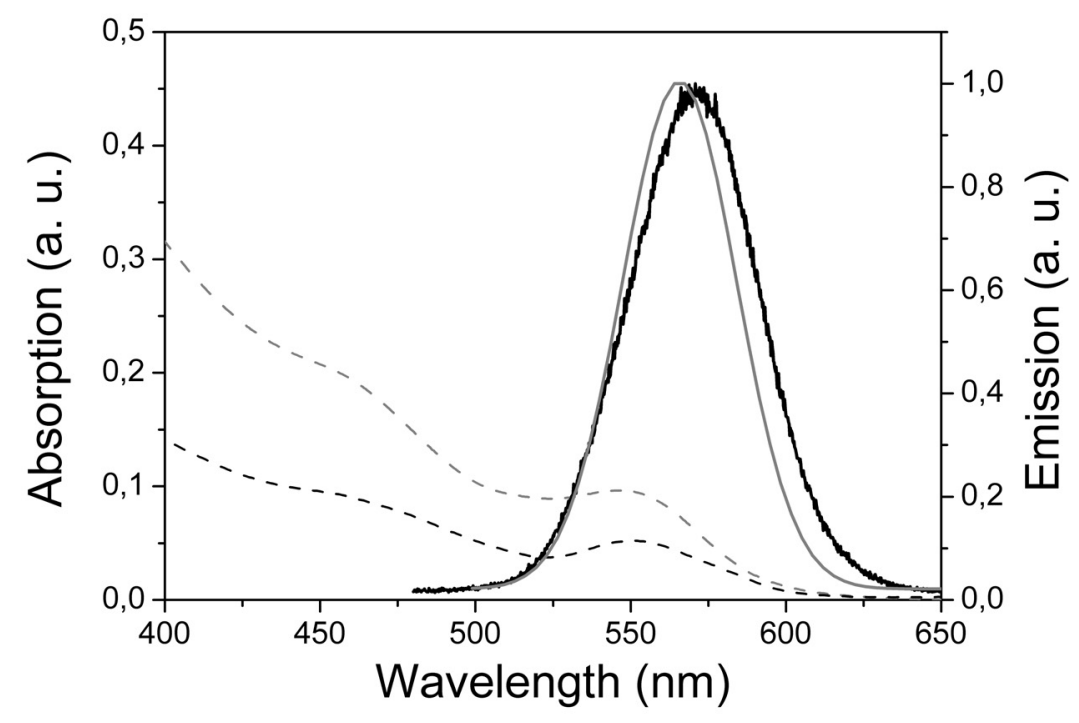

Figure 5.1. Absorption (dashed line) and normalized emission (solid line) spectra of QDs in chloroform (grey) and in water (black).

The lowest energy absorption bands of the spectra of both compounds peak at a similar wavelength $\sim 550 \mathrm{~nm}$, although the spectrum of the MAA-coated QDs is slightly broader. The emission peak of the MAA-coated QDs $(\sim 570 \mathrm{~nm})$ is slightly shifted with respect to that observed for the TOPO-coated QDs $(\sim 565 \mathrm{~nm})$. It is known that exchanging the TOPO ligands on the surface of QDs with thiols (or other types of ligands) may cause changes in the absorption and emission profiles [24]. Nevertheless, the slight differences observed here could be related simply to the changes in the dielectric environment of the QDs (different solvents) or to the presence of charges on the QD surface. The size of the CdSe core calculated from the first absorption peak is $\sim 3 \mathrm{~nm}[21]$.

The PNIPAM polymer films were obtained by an "iniferter-type" controlled radical polymerization. Iniferters are initiators of radical polymerization that also act as propagation and termination agents [20]. In this study, we synthesized a disulfide-based initiator, DTCA, to graft the polymer chains to the gold substrate [20]. (The structure of the initiator is shown in Figure 5.2.a). In context of the present work there are three attractive features which such type of polymerization may provide. First, the initiator was shown to bind effectively to the gold surface, while retaining its polymerization initiating functions [20]. This feature allows us to grow polymeric chains directly form the gold substrate. 
a
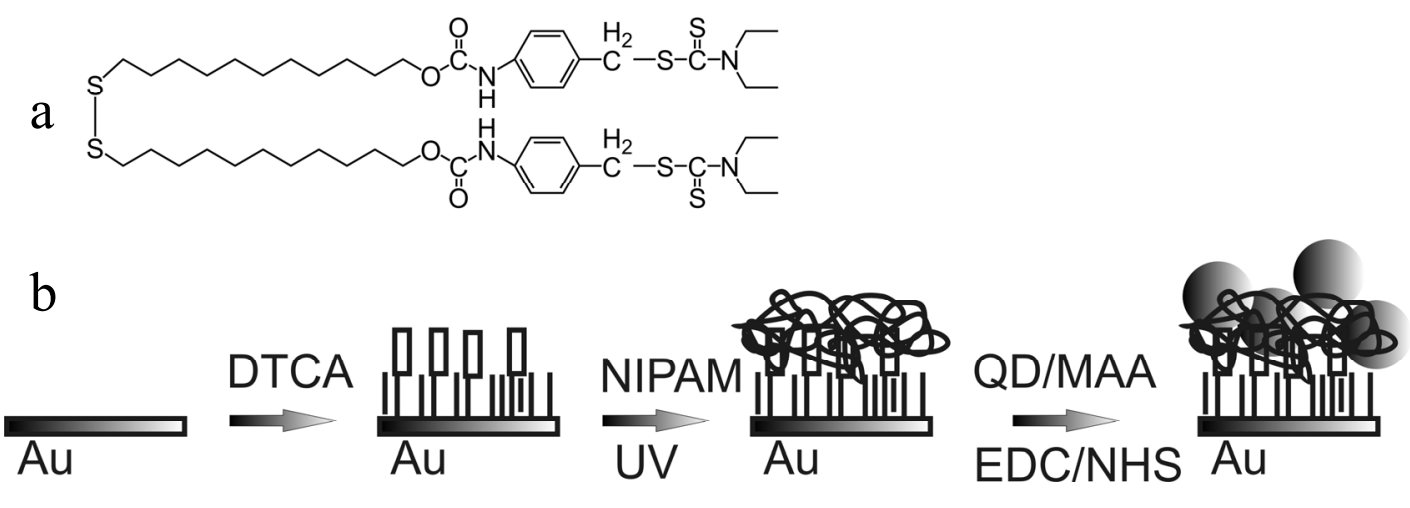

Figure 5.2. a) The chemical structure of the iniferter DTCA, b) scheme of the gold surface functionalization with PNIPAM and QDs. The polymer chains are grown directly from the $\mathrm{Au}$ surface by UV initiated controlled radical polymerization. The radical chain ends are transformed to amines for coupling to $\mathrm{COOH}$ modified QDs (see experimental section).

Second, controlled living radical polymerization allows us to set the molar mass of the polymer, and therefore the resulting film thickness. Finally, the reactive diethyldithiocarbamyl group remaining at the end of the growing PNIPAM chain can be exchanged for another chemical group to introduce functionality for further coupling with QDs [25]. A more detailed description and characterization of the PNIPAM brushes can be found in [20]. In order to couple the QDs to the PNIPAM chain-ends we exchanged the radical chain ends of PNIPAM with 4-amino-TEMPO groups. EDC-assisted coupling between carboxylic acids and primary amines permits linking the functionalized PNIPAM chain ends to the MAA-coated QDs, resulting in an amide bond formation [26]. Addition of NHS improves the efficiency of the reaction by increased stability of the NHS ester intermediate. The general experimental scheme is presented in Figure 5.2b.

Fluorescence imaging of a sample with a removed initiator layer (by scratching it from the glass surface) showed that, after washing the substrate, emission originated only from the areas where the brush has been grown and the quantum dots were covalently attached to the chain ends (Figure 5.3). 


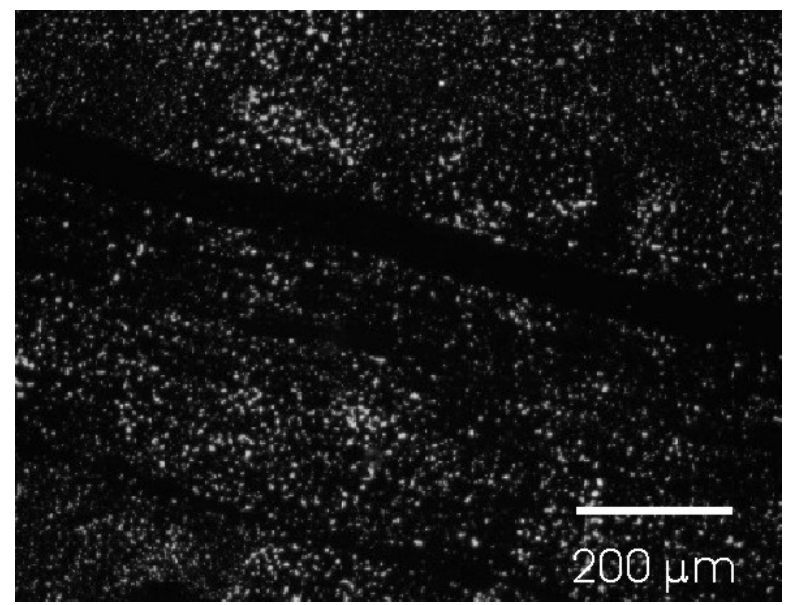

Figure 5.3. Scratched surface of iniferter coated gold substrate. The attachment of QDs occurs only in the non-scratched portion of the surface, where the iniferter-terminated chain ends are present.

The surface of the QD/PNIPAM layer was investigated by AFM. Figure 5.4 shows AFM height images of the PNIPAM brushes obtained before and after the attachment of QDs. Upon attachment of the QDs the surface roughness and variations in height increase significantly, indicating the presence of the nanoparticles on top of the PNIPAM brushes. The root mean square $(\mathrm{rms})$ roughness of the surface calculated before $(0.8 \mathrm{~nm})$ and after $(2.3 \mathrm{~nm})$ QD attachment is in good agreement with the results obtained by Gupta et al., where the rms values for PAA brushes without immobilized QDs was $0.7 \mathrm{~nm}$, and $2.2 \mathrm{~nm}$ after QD attachment [7]. AFM results suggest that the amino end-functionalized PNIPAM brushes can serve as efficient layers for covalent immobilization of carboxy-terminated QDs.
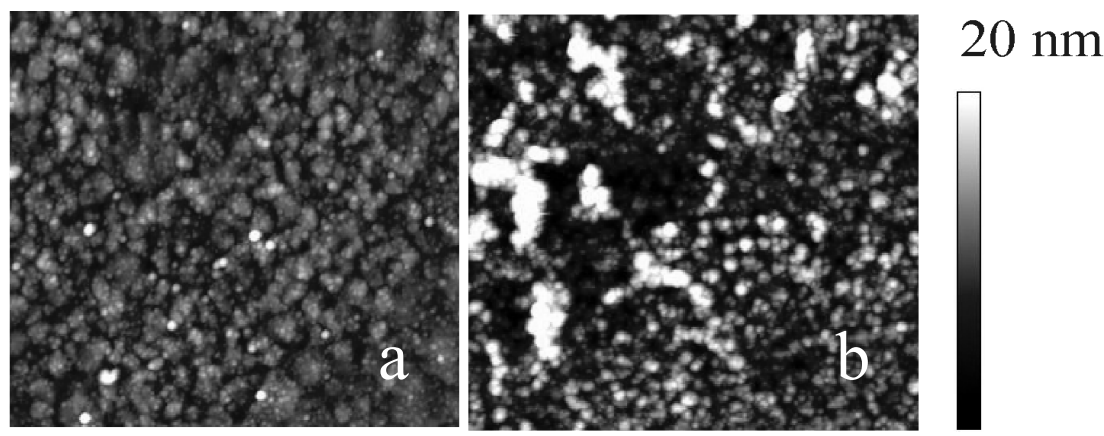

Figure 5.4. AFM height images of PNIPAM brush layer on gold before (a) and after (b) attachment of the QDs. (Scan size: 1 x $1 \mu \mathrm{m}$ ). 
The central analysis of the hybrid polymer/QD platform was performed via temperature variation. The PNIPAM brushes were shown previously to display LCST behaviour similar to that of linear polymers or polymer networks [25, 27, 28]. Upon increasing the temperature and crossing the LCST, the polymer chains collapse, reducing the thickness of the polymer layer on the substrate. Upon decreasing the temperature below the LCST, the brush layer swells in water to restore the original thickness. This behaviour suggests that in the present study, in which a layer of QDs sits on top of the brush layer, it should therefore be possible to modulate the distance of the nanoparticles to the gold substrate. The temperature-induced collapse and swelling will result in a modulation of the distance between the QDs and gold substrate, which will be reflected in the luminescence properties.

The temperature response of the QD/PNIPAM luminescence upon heating and cooling the system in an aqueous environment is shown in Figure 5.5 (left). The temperature was varied from $25^{\circ} \mathrm{C}$ to $50^{\circ} \mathrm{C}$. During heating, a decrease in the luminescence intensity was observed. Upon crossing the LCST and reaching $50^{\circ} \mathrm{C}$, almost no luminescence $(94 \%$ less intensity than initially) could be observed from the sample (Figure 5.5(2)). Subsequently, the heating was switched off and the luminescence intensity could be observed to recover $(68 \%$ of the initial intensity) (Figure 5.5(3)).
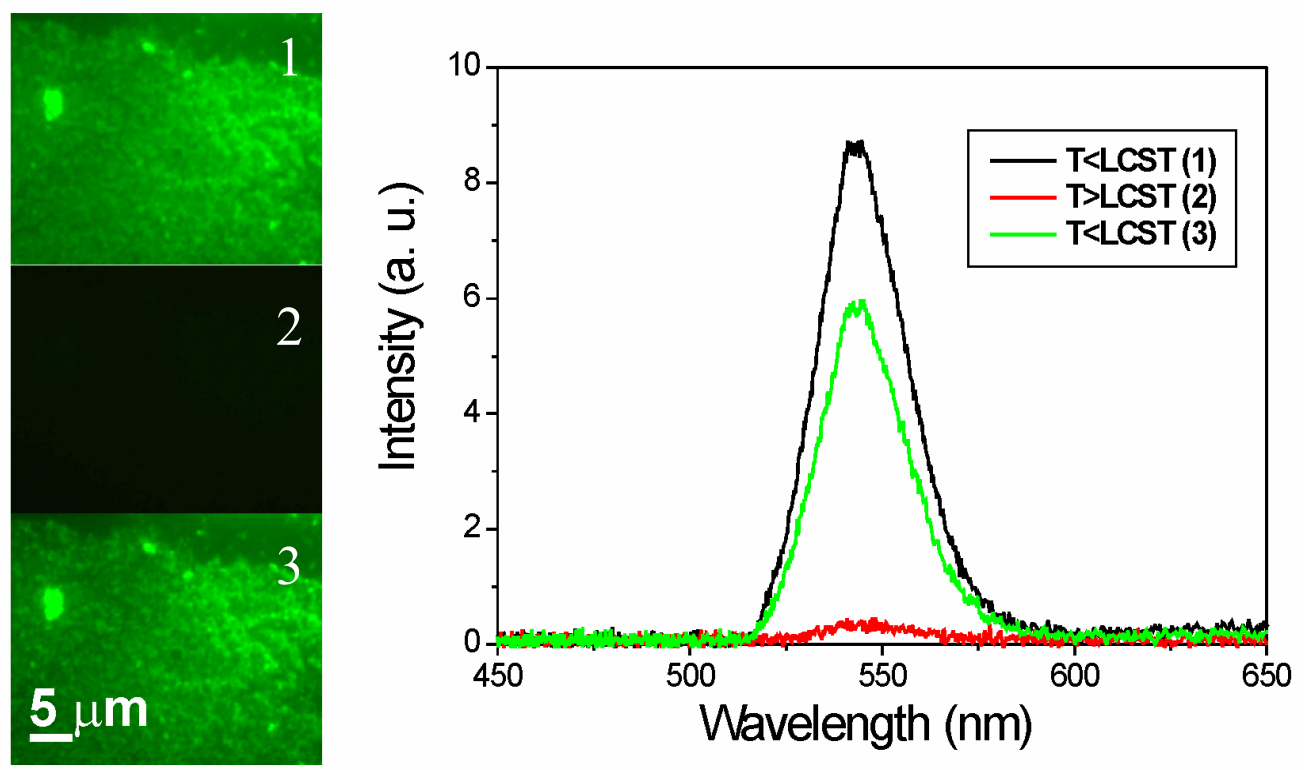

Figure 5.5. Left: Luminescence images of $\mathrm{Au} / \mathrm{PNIPAM} / \mathrm{QD}$ assembly obtained at $25^{\circ} \mathrm{C}(1)$, after heating to $50^{\circ} \mathrm{C}(2)$, and after cooling back to $25{ }^{\circ} \mathrm{C}$ (3). Right: Spectra measured at below the LCST $(1,3)$ and above the LCST (2), showing the quenching and recovery behaviour. 
Corresponding spectral measurements obtained for temperatures below and above the LCST are shown in Figure 5.5 (right). The emission peak upon excitation of the sample with a $469 \mathrm{~nm}$ laser line is centred around $547 \mathrm{~nm}$ and no apparent shift is observed comparing to the emission peak of MAA coated QDs in water. The incomplete recovery of the fluorescence upon cooling the sample below its LCST could be explained by the hysteresis in the swelling/deswelling processes, which are usually observed in PNIPAM-related systems due to intra- and inter-chain interactions formed in the collapsed state at T $>$ LCST [27]. Such hysteresis in the first cycle was reported to increase for thin hydrogel films due to constrain imposed by the crosslink points [27]. In our system, the immobilized QDs might act as additional surface crosslink points influencing the hysteresis.

The hybrid polymer/QD system shows a remarkable ON/OFF switching and good recovery of the emission spectra, indicating high photostability of both the QDs and assembly during prolonged heating and irradiation. In order to elucidate the mechanism of switching, we consider a number of possible scenarios. There is a possibility that the QDs could be incorporated into the polymer layer by swelling and hydrogen bond formation with PNIPAM. However, at T>LCST, PNIPAM becomes hydrophobic and would expel the noncovalently attached QDs. The good recovery of the fluorescence intensity after each heating/cooling cycle indicates that only the emission originating from the covalently bound QDs is observed. Additionally Snaith et al [17] reported that QDs with diameters larger than $3 \mathrm{~nm}$ form bilayer structures on polymer brushes rather than infiltrate the brush interior due to unfavourable entropic penalties. Hence, we can conclude that the observed intensity changes are related to changes in the $\mathrm{Au} / \mathrm{PNIPAM} / \mathrm{QD}$ assembly that originate from the temperature induced thickness variations of the PNIPAM layer.

The photoluminescence of QDs embedded in various matrices (also polymers) was found to be temperature dependent and to decrease linearly by $1.3 \% /{ }^{\circ} \mathrm{C}$ with increasing temperature [29]. Given the temperature range investigated here, this effect would correspond to around $30 \%$ of decrease in intensity, which is far below the quenching observed in our study.

We have additionally investigated the effects of the temperature on the emission of the bare QDs in solution and found a decrease in luminescence only around 15\%, much below the $87 \%$ decrease observed when the QDs are coupled to the PNIPAM brushes grown from the gold substrate (Figure 5.6). 


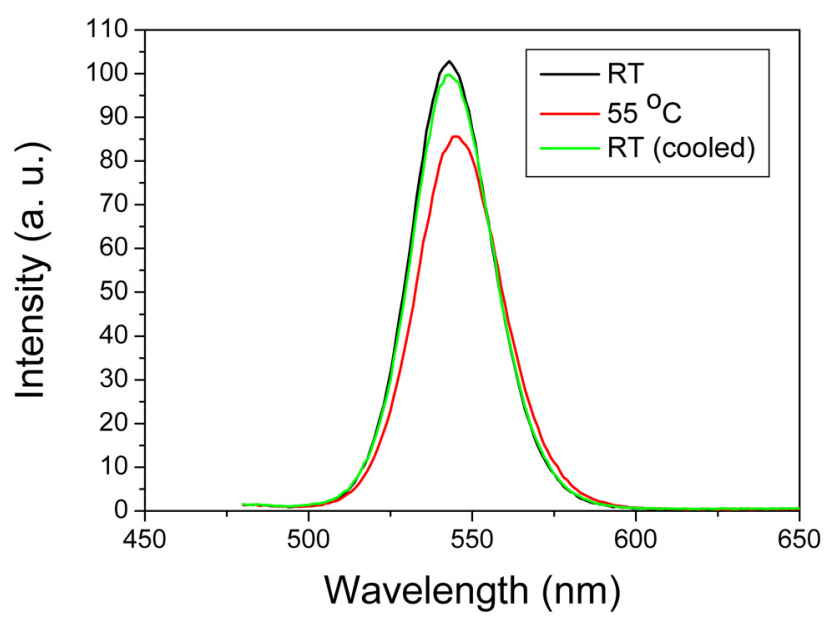

Figure 5.6. Luminescence emission of MAA coated QDs in solution as a function of temperature. The emission was first measured at $20{ }^{\circ} \mathrm{C}(\mathrm{RT})$, luminescence intensity drops and slightly red shifts upon heating using a Peltier thermoelectric cuvette holder $\left(55^{\circ} \mathrm{C}\right)$ by $15 \%$ and recovers on cooling the solution to $20{ }^{\circ} \mathrm{C}$ (RT-cooled).

The most plausible explanation for the observed emission changes is quenching of the luminescence of the QDs by the $\mathrm{Au}$ substrate (Figure 5.7). In general, the interaction between QDs and metals may result in enhanced photoluminescence [30,31] or in increased nonradiative decay rates due to energy transfer via electromagnetic interaction [31, 32].

\section{CdSe/ZnS QDs}

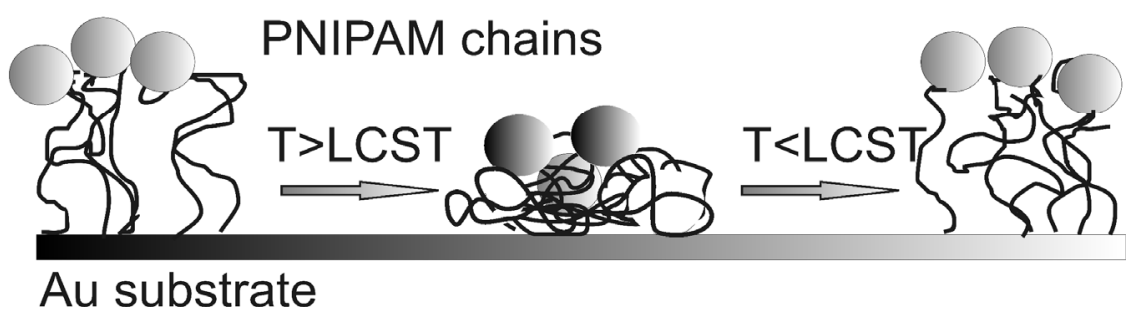

Figure 5.7. Proposed structure and temperature-dependent behaviour of the $\mathrm{Au} / \mathrm{PNIPAM} / \mathrm{QD}$ assembly. At $\mathrm{T}<\mathrm{LCST}$ the polymer brushes are hydrophilic and are in the extended configuration. The quenching of the emission by the gold substrate is limited. At T $>$ LCST the PNIPAM chains collapse reducing the brush film thickness and bringing the QDs closer to the gold surface where pronounced quenching takes place. The transition is reversible and upon cooling the PNIPAM brushes swell in water and the luminescence is recovered. 
Photoluminescence enhancement is not expected to be pronounced in our study as we excited the QDs at frequencies far from the gold surface plasmon resonance. Quenching of the luminescence by energy transfer to metals is a short-distance (in the $10 \mathrm{~nm}$ range) effect, which is modulated by the spectral overlap and distance between the energy donor and acceptor, and which is sensitive to surface roughness and to the local environment. In our system the distance between the QDs and gold is modulated via temperature-induced swelling and deswelling of the PNIPAM layer. This in turn influences the decay rates and results in luminescence quenching. The photoluminescence decay rate is a sum of the internal decay rates (radiative and nonradiative) of the QD and the energy transfer rate from the QD to the metal. To elucidate the exact mechanism for the quenching a more detailed study including life time measurements shall be performed in the future.

\subsection{Conclusions}

We have demonstrated a thermo-responsive metal/polymer/quantum dot platform based on PNIPAM brushes grafted from gold substrate using an iniferter-type polymerization, and QDs covalently attached to the PNIPAM chain ends. The EDC/NHS chemistry was shown to be efficient in coupling of $\mathrm{COOH}$ functionalized QDs to amineterminated PNIPAM brushes. Luminescence imaging and, luminescence spectra measurements showed that upon increasing the temperature above the LCST of PNIPAM the QD emission is quenched. When decreasing the temperature below the LCTS, recovery of the luminescence can be observed. The results obtained are consistent with temperature modulated thickness of the PNIPAM brushes and quenching of the nanoparticles luminescence by the gold substrate. The Au/PNIPAM/QD system reported here may be explored in sensing and surface thermometry. The iniferter-type polymerization would also allow one to control the film thickness and improve the sensitivity and time response of the system.

\subsection{References}

1. Tokareva, I., Minko, S., Fendler, J.H., Hutter, E. J Am Chem Soc, 2004. 126(49): p. 15950.

2. Mack, N.H., Wackerly, J.W., Malyarchuk, V., Rogers, J.A., Moore, J.S.; Nuzzo, R.G., Nano Lett, 2007. 7(3): p. 733.

3. Zhao, B.B., Brittain, W.J., Prog Pol Sci, 2000. 25(5): p. 677. 
4. Advincula, R.C., Brittain, W.J., Caster, K.C., Rühe, J. Polymer Brushes: Synthesis, Characterization, Applications. 1st ed. 2004, Weinheim: Wiley-VCH.

5. Luzinov, I., Minko, S., Tsukruk, V.V., Prog Pol Sci, 2004. 29(7): p. 635.

6. Ionov, L., Sapra, S., Synytska, A., Rogach, A.L., Stamm, M., Diez, S., Adv Mater, 2006. 18(11): p. 1453.

7. Gupta, S., Uhlmann, P., Agrawal, M., Lesnyak, V., Gaponik, N., Simon, F., Stamm, M., Eychmüller, A., J Mater Chem, 2008. 18(2): p. 214.

8. Pardo-Yissar, V., Bourenko, T., Wasserman, J., Willner, I., Adv Mater, 2002. 14(9): p. 670 .

9. Mitsuishi, M., Koishikawa, Y., Tanaka, H., Sato, E., Mikayama, T., Matsui, J., Miyashita, T., Langmuir, 2007. 23(14): p. 7472.

10. Ionov, L., Synytska, A., Diez, S. ,Adv Funct Mater, 2008. 18(10): p. 1501.

11. Alexander, C., Shakesheff, K.M. , Adv Mater, 2006. 18(24): p. 3321.

12. Schild, H.G., Prog Pol Sci, 1992. 17(2): p. 163.

13. Bruchez, M., Jr., Moronne, M., Gin, P., Weiss, S., Alivisatos, A.P., Science, 1998. 281(5385): p. 2013.

14. Chan, W.C., Maxwell, D.J., Gao, X., Bailey, R.E., Han, M., Nie, S., Curr Opin Biotechnol, 2002. 13(1): p. 40.

15. Shavel, A., Gaponik, N., Eychmuller, A. ,Chem Phys Chem, 2005. 6(3): p. 449.

16. Ostrander, J.W., Mamedov, A.A., Kotov, N.A., J Am Chem Soc, 2001. 123(6): p. 1101.

17. Snaith, H.J., Whiting, G.L., Sun, B., Greenham, N.C., Huck, W.T.S., Friend, R.H., Nano Lett, 2005. 5(9): p. 1653.

18. Janczewski, D., Tomczak, N., Khin, Y.W., Han, M.Y., Vancso, G.J., Eur Polym J, 2009, 45: p. 3.

19. Chan, W.C.W., and Nie, S., Science, 1998. 281(5385): p. 2016.

20. Benetti, E.M., Zapotoczny, S., Vancso, G.J., Adv Mater, 2007. 19(2): p. 268.

21. Yu, W.W., Qu, L., Guo, W., Peng, X., Chem Mater, 2003. 15(14): p. 2854.

22. Aldana, J., Wang, Y.A., Peng, X., J Am Chem Soc, 2001. 123(36): p. 8844.

23. Wuister, S.F., Swart, I., van Driel, F., Hickey, S.G., de Mello Donega, C., Nano Lett, 2003. 3(4): p. 503.

24. Mitchell, G.P., Mirkin, C.A., Letsinger, R.L., J Am Chem Soc, 1999. 121(35): p. 8122.

25. Matsuda, T. and Ohya, S., Langmuir, 2005. 21(21): p. 9660. 
26. Cameron, P.J., Zhong, X., Knoll, W., J Phys Chem C, 2007. 111(28): p. 10313.

27. Cao, Z., Du, B., Chen, T., Li, H., Xu, J., Fan, Z., Langmuir, 2008. 24(10): p. 5543.

28. Tagit, O., Tomczak, N., Vancso, G.J., Small, 2008. 4(1): p. 119.

29. Walker, G.W., Sundar, V.C., Rudzinski, C.M., Wun, A.W., Bawendi, M.G., Nocera, D.G., Appl Phys Lett, 2003. 83(17): p. 3555.

30. Kulakovich, O., Strekal, N., Yaroshevich, A., Maskevich, S., Gaponenko, S., Nabiev, I., Woggon, U., Artemyev,M., Nano Lett, 2002. 2(12): p. 1449.

31. Matsuda, K., Ito, Y., Kanemitsu, Y., Appl Phys Lett, 2008. 92(21): p. 211911.

32. Ueda, A., Tayagaki, T., Kanemitsu, Y., Appl Phys Lett, 2008. 92(13): p. 133118. 


\section{Chapter 6}

\section{Thermoresponsive quantum dot/PNIPAM assemblies*}

Synthesis, characterization, and application of novel thermoresponsive polymeric coatings for Quantum Dots (QDs) is presented. Comb-copolymers featuring hydrophobic alkyl groups, carboxylic groups and poly(N-isopropylacrylamide) (PNIPAM) side chains with molar masses ranging from $1000 \mathrm{~g} / \mathrm{mol}$ to $25400 \mathrm{~g} / \mathrm{mol}$ were obtained. The amphiphilic combcopolymers were shown to efficiently transfer the QDs to aqueous media. The PNIPAMcoated QD materials display a lower critical solution temperature (LCST). The absorbance, luminescence emission, size of the assemblies, and electrophoretic mobility were followed as a function of temperature and the reversibility of the temperature induced changes is demonstrated by cyclic heating and cooling.

\footnotetext{
* Parts of this chapter have been submitted for publication to: Tagit, O., Jańczewski, D., Tomczak, N., Han, MY., Herek, J.L., Vancso, G.J. Thermoresponsive quantum dot/PNIPAM assemblies Macromolecules.
} 



\subsection{Introduction}

Stimulus responsive organic-inorganic hybrid materials find applications in drug delivery [1], bionanotechnology [2, 3] and sensing [4], among other areas. The organic part of the hybrids is made of so-called "smart" polymers, i.e., polymers that undergo conformational changes in response to external stimuli such as temperature [5], $\mathrm{pH}$ [6], or solvent [7]. Poly( $N$-isopropyl acrylamide), PNIPAM, is probably the best-known and the most-studied thermoresponsive polymer exhibiting a lower critical solution temperature (LCST). Below the critical transition, the polymer is hydrophilic and disperses well in aqueous solutions. Above the $\operatorname{LCST}\left(\sim 32^{\circ} \mathrm{C}\right.$ for PNIPAM), the polymer becomes hydrophobic. Thus, at or above the LCST the polymer deswells and the chains collapse [8]. The LCST behavior of PNIPAM has been widely explored for drug delivery [9] and sensing applications [10]. The inorganic part of the hybrids may serve not only as a scaffold for the organic part but may also display functionalities such as magnetic or luminescent properties. Regarding hybrid materials, thermoresponsive polymeric coatings have been applied to magnetic iron oxide [1, 3, 4, 11-14], gold [15-22] and silica nanoparticles [18, 23-25].

Semiconductor nanoparticles such as quantum dots (QDs), gained much attention as luminescent materials due to their unique composition and size-tunable properties, broad excitation and narrow emission spectra, and high photochemical stability [26]. Combining QDs with thermoresponsive coatings, such as PNIPAM, offers many attractive possibilities, but has not yet been widely explored [27]. Hybrid thermoresponsive polymer/QD assemblies have previously been prepared by grafting end-functionalized polymer chains [28, 29], surface initiated polymerization [30], hydrogen bond formation [31], or solvent induced encapsulation [32]. Alternatively, one can employ amphiphilic polymers to coat the surface of hydrophobic QDs [33-35]. A major advantage of amphiphilic coatings is the ability to introduce diverse functionalities at the QD surface. Coatings with grafted polymer side chains can also be prepared, and a one-step coating procedure may be employed to prepare QDs with grafted polymer chains. Such coating would endow the QDs with water-dispersability and thermoresponsiveness. Application of amphiphilic coatings with thermoresponsive side polymer chains for QDs however was not studied to date. The limits imposed on the molar mass of the polymer side-chains for effective transfer of the QDs to water using amphiphilic polymers are also not known. The molar mass of the thermoresponsive polymer grafted onto the nanoparticles may in addition influence the thermally induced collapse of the hybrids 
[20]. Thus, some consideration should be given to the molar mass of the thermoresponsive polymeric coatings on the QD surface.

In this chapter, we show that aqueous dispersions of hybrid PNIPAM/QD assemblies can be prepared using TOPO-coated QDs and engineered polymeric coatings. The coatings consist of an amphiphilic polymer bearing PNIPAM side chains. CdSe/ZnS QDs are grafted with PNIPAM chains with molar masses of $1.0 \times 10^{3}, 10.8 \times 10^{3}$, and $25.4 \times 10^{3} \mathrm{~g} / \mathrm{mol}$ and are transferred into water. The hybrid materials exhibit a well-pronounced LCST behavior. Above the LCST the PNIPAM chains on the surface of the QDs become hydrophobic and collapse. However, the presence of carboxylate groups on the amphiphilic coating prevents the nanoparticles from aggregating. As a result, higher electrophoretic mobility is observed above LCST. The transition is fully reversible, as observed during a number of heating and cooling cycles.

\subsection{Experimental section}

Core-shell CdSe/ZnS QDs were synthesized as reported previously [35]. Aminoterminated PNIPAM ( $\mathrm{NH}_{2}$-PNIPAM) with three different molar masses $\left(\mathrm{M}_{\mathrm{w}}\right) 1.0 \times 10^{3}, 10.8$ $\mathrm{x} 10^{3}$, and $25.4 \times 10^{3} \mathrm{~g} / \mathrm{mol}$ were purchased from Polymer Source Inc. (Canada). $n$ octylamine, $N, N$-diisopropylethylamine (DIPEA), poly(isobutylene-alt-maleic anhydride) $\left(\mathrm{M}_{\mathrm{w}}=6000 \mathrm{~g} / \mathrm{mol}\right)$ and other chemicals were purchased from Sigma-Aldrich.

Synthesis of polymer 1 (P1). $0.4 \mathrm{~mL}$ of $n$-octylamine and $1.0 \mathrm{~mL}$ of DIPEA, were added to $300 \mathrm{~mL}$ of a THF solution containing $0.35 \mathrm{~g}$ of isobutylene-alt-maleic anhydride and stirred until a clear solution was obtained. $1.00 \mathrm{~g}$ of PNIPAM $\left(1 \times 10^{3} \mathrm{~g} / \mathrm{mol}, \mathrm{M}_{\mathrm{w}} / \mathrm{M}_{\mathrm{n}}\right.$ : 1.8) was added to the solution and the reaction was left to proceed for 12 hours at $50{ }^{\circ} \mathrm{C}$. Subsequently THF was evaporated and the resulting material was suspended in water with small excess of $\mathrm{NaOH}(\mathrm{pH}=11)$ with respect to the carboxylic groups on the polymer backbone. After evaporation of water and DIPEA, the remaining residue was dissolved in water and dialyzed against $0.01 \mathrm{M} \mathrm{NaOH}$ and pure water for a week. Evaporation of water by freeze-drying resulted with $0.4877 \mathrm{~g}$ of polymer $\mathbf{P 1}$ as white powder. The polymer composition was determined by ${ }^{1} \mathrm{H}$ NMR : $\delta_{\mathrm{H}}\left(400 \mathrm{MHz} ; \mathrm{D}_{2} \mathrm{O}\right): 7.35$ (9 H, br), $3.93(153 \mathrm{H}$, br), $3.77-2.45$ (112 H, m), 2.45 - 1.85 (255 H, m), 1.85 - 1.47 (350 H, br), 1.38 (253 H, br), 1.20 (921 H, br), $1.15-0.82(284 \mathrm{H}, \mathrm{m})$. 
Synthesis of polymer 2 (P10). $0.06 \mathrm{~mL}$ of $n$-octylamine and $0.15 \mathrm{~mL}$ of DIPEA, were added to $300 \mathrm{~mL}$ of a THF solution containing $0.14 \mathrm{~g}$ of isobutylene-alt-maleic anhydride and stirred until a clear solution was obtained. $1.00 \mathrm{~g}$ of PNIPAM $\left(10.8 \times 10^{3} \mathrm{~g} / \mathrm{mol}, \mathrm{M}_{\mathrm{w}} / \mathrm{M}_{\mathrm{n}}\right.$ : 1.55) was added to the solution and the reaction was left to proceed for 12 hours at $50{ }^{\circ} \mathrm{C}$. Subsequently THF was evaporated and the resulting material was suspended in water with small excess of $\mathrm{NaOH}(\mathrm{pH}=11)$ with respect to the carboxylic groups on the polymer backbone. After evaporation of water and DIPEA, the remaining residue was dissolved in water and dialyzed against $0.01 \mathrm{M} \mathrm{NaOH}$ and pure water for a week. Evaporation of water by freeze-drying resulted with $1.0708 \mathrm{~g}$ of polymer P10 as white powder. The polymer composition was determined by ${ }^{1} \mathrm{H}$ NMR: $\delta_{\mathrm{H}}\left(400 \mathrm{MHz} ; \mathrm{D}_{2} \mathrm{O}\right): 7.34(7 \mathrm{H}, \mathrm{br}), 3.93(281 \mathrm{H}$, br), $3.79-2.45(102 \mathrm{H}, \mathrm{m}), 2.45-1.88(362 \mathrm{H}, \mathrm{m}), 1.85-1.42$ (539 H, br), 1.33 (257 H, br), $1.18(1687 \mathrm{H}, \mathrm{br}), 1.15-0.82(285 \mathrm{H}, \mathrm{m})$.

Synthesis of polymer 3 (P25). $0.12 \mathrm{~mL}$ of $n$-octylamine and $0.3 \mathrm{~mL}$ of DIPEA, were added to $300 \mathrm{~mL}$ of a THF solution containing $0.31 \mathrm{~g}$ of isobutylene-alt-maleic anhydride and stirred until a clear solution was obtained. $1.00 \mathrm{~g}$ of PNIPAM $\left(25.4 \times 10^{3} \mathrm{~g} / \mathrm{mol}, \mathrm{M}_{\mathrm{w}} / \mathrm{M}_{\mathrm{n}}\right.$ : 2.49) was added to the solution and the reaction was left to proceed for 12 hours at $50{ }^{\circ} \mathrm{C}$. Subsequently THF was evaporated and the resulting material was suspended in water with small excess of $\mathrm{NaOH}(\mathrm{pH}=11)$ with respect to the carboxylic groups on the polymer backbone. After evaporation of water and DIPEA, the remaining residue was dissolved in water and dialyzed against $0.01 \mathrm{M} \mathrm{NaOH}$ and pure water for a week. Evaporation of water by freeze-drying resulted with $1.318 \mathrm{~g}$ of polymer $\mathbf{P 2 5}$ as white powder. The polymer composition was determined by ${ }^{1} \mathrm{H}$ NMR: $\delta_{\mathrm{H}}\left(400 \mathrm{MHz} ; \mathrm{D}_{2} \mathrm{O}\right): 7.33(9 \mathrm{H}, \mathrm{br}), 3.92(92 \mathrm{H}, \mathrm{br})$, $3.79-2.45$ (68 H, m), $2.45-1.84(167 \mathrm{H}, \mathrm{m}), 1.84-1.42$ (213 H, br), 1.32 (179 H, br), 1.17 (567 H, br), $1.15-0.75(266 \mathrm{H}, \mathrm{m})$.

Coating of QDs with amphiphilic polymers. $3 \mathrm{mg}$ of purified QDs were suspended in $3 \mathrm{~mL}$ of THF. 30, 60 and $30 \mathrm{mg}$, of polymers P1, P10 and P25, respectively, were dissolved in 2 $\mathrm{mL}$ of water. The polymer and QD solutions were mixed while purging with argon. Following the evaporation of THF and half of the volume of water, the solutions were diluted with $2 \mathrm{~mL}$ of water. The resulting aqueous solutions of polymer coated QDs R1, R10 and R25 were filtered through a $0.22 \mu \mathrm{m}$ MILEX PES membrane filter and centrifuged at 10000 rpm for 30 minutes. 
Characterization methods. ${ }^{1} \mathrm{H}$ NMR spectra were obtained using a Bruker spectrometer (DRX $400 \mathrm{MHz}$ ). UV-Vis absorption was recorded using a Shimadzu spectrophotometer (UV-1601). A Cary Eclipse spectrofluorometer with a Peltier thermoelectric cuvette holder was used to obtain the luminescence spectra of the QD solutions. Particle size and surface charge measurements were performed with a Zetasizer Nano ZS (Malvern Instruments, Malvern, UK) using light with a wavelength of $633 \mathrm{~nm}\left(4 \mathrm{~mW}, 173^{\circ}\right.$ measurement angle). Dynamic light scattering (DLS) is a powerful tool to characterize the effective Stokes hydrodynamic size of micro- or nanoparticles, including polymers in solution, and the electrophoretic mobility of charged particles [36]. In principle, if absorbing (and/or luminescent) particles are present in the scattering volume, a complex refractive index must be used in the Rayleigh formalism of light scattering. Chromophore absorption causes intensity loss, heating, and convection effects in the scattering volume. The (incoherent, scattering angle independent) emission intensity is added to the scattered intensity measured. As a result, the scattering function becomes distorted. However, in DLS essentially only local heating can perturb the experiment in routine characterization studies, which can be reduced by low concentration of the particles, low incident light intensity (at the cost of experimental sensitivity), and using a heat dissipating experimental geometry [37]. If proper precautions are taken, DLS can be used with success to characterize the hydrodynamic dimensions, electrophoretic mobility and stability of water soluble QDs capped with various surface ligands as shown by Mattoussi et al. [38]. In our work we used a low intensity laser and a Malvern particle sizer to characterize the QD-PNIPAM assemblies.

\subsection{Results and discussion}

Core-shell CdSe/ZnS QDs were synthesized in the presence of trioctylphosphine oxide (TOPO), triphenylphosphine (TOP) and $n$-hexadecylamine according to the reported procedure [35]. The size of the QDs was controlled by the reaction temperature and the time elapsed until the shell material was added to the reaction mixture after the initial core formation. Reaction at $235{ }^{\circ} \mathrm{C}$ for 30 minutes resulted in red-emitting QDs with a first absorption peak located at $620 \mathrm{~nm}$ and with a narrow emission spectrum (35 nm full width at half maximum) with a peak located at $638 \mathrm{~nm}$ (Figure 6.1). The core size of the QDs was estimated to be equal to $\sim 5.6 \mathrm{~nm}$ by using the reported empirical equation [39]. 


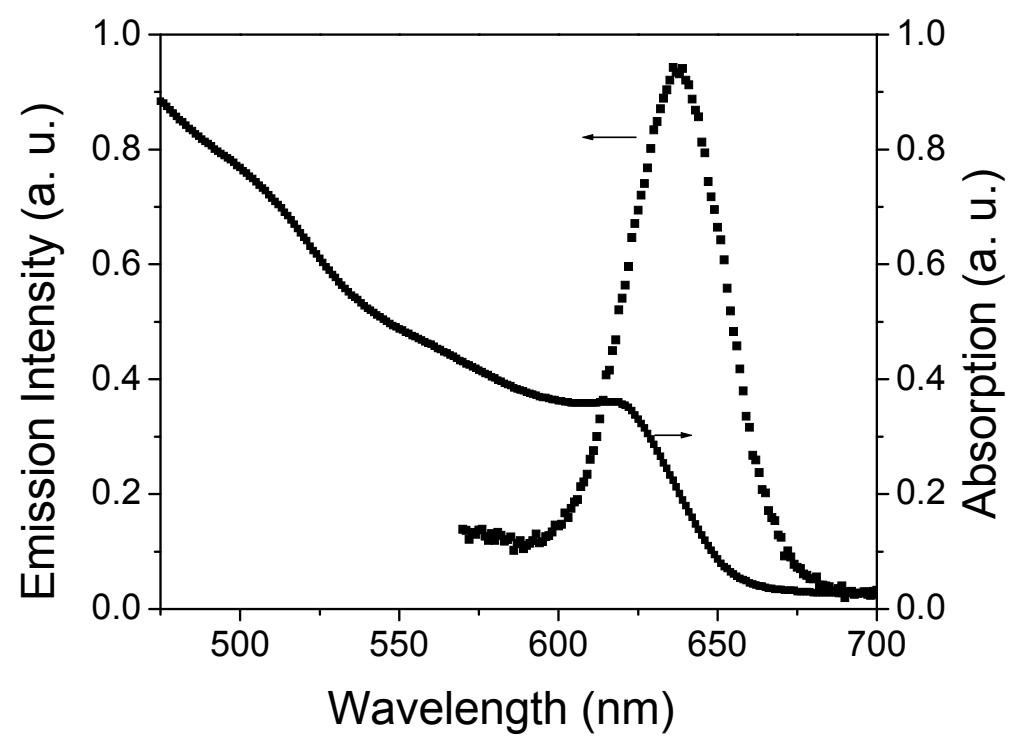

Figure 6.1. Normalized absorption and emission spectra of TOPO-coated CdSe/ZnS QDs in chloroform solution.

Our strategy to fabricate QD materials with temperature responsiveness is based on the design of suitable polymeric coatings for QDs, which include functional parts composed of PNIPAM. To this end, we have employed an amphiphilic polymer structure, which was already reported to efficiently coat hydrophobically-functionalized QDs [35]. A number of different functionalities including bulky side groups and longer PEG chains appended to the amphiphilic polymer were shown not to preclude the transfer of the QDs into water. The synthesis of functionalized amphiphilic polymers is based on anhydride ring opening with amine-functionalized molecules in presence of an amine catalyst. For the polymer to serve as an efficient amphiphilic coating one needs to include hydrophobic side groups for interactions with the hydrophobic ligands on the QD surface, and hydrophilic groups to attain colloidally stable suspension of the nanoparticles in water. Reaction of the anhydride polymer backbones with $n$-octylamine and amine-terminated PNIPAM of different molar masses resulted in a series of amphiphilic polymers with a general structure shown in Figure 6.2. 
a
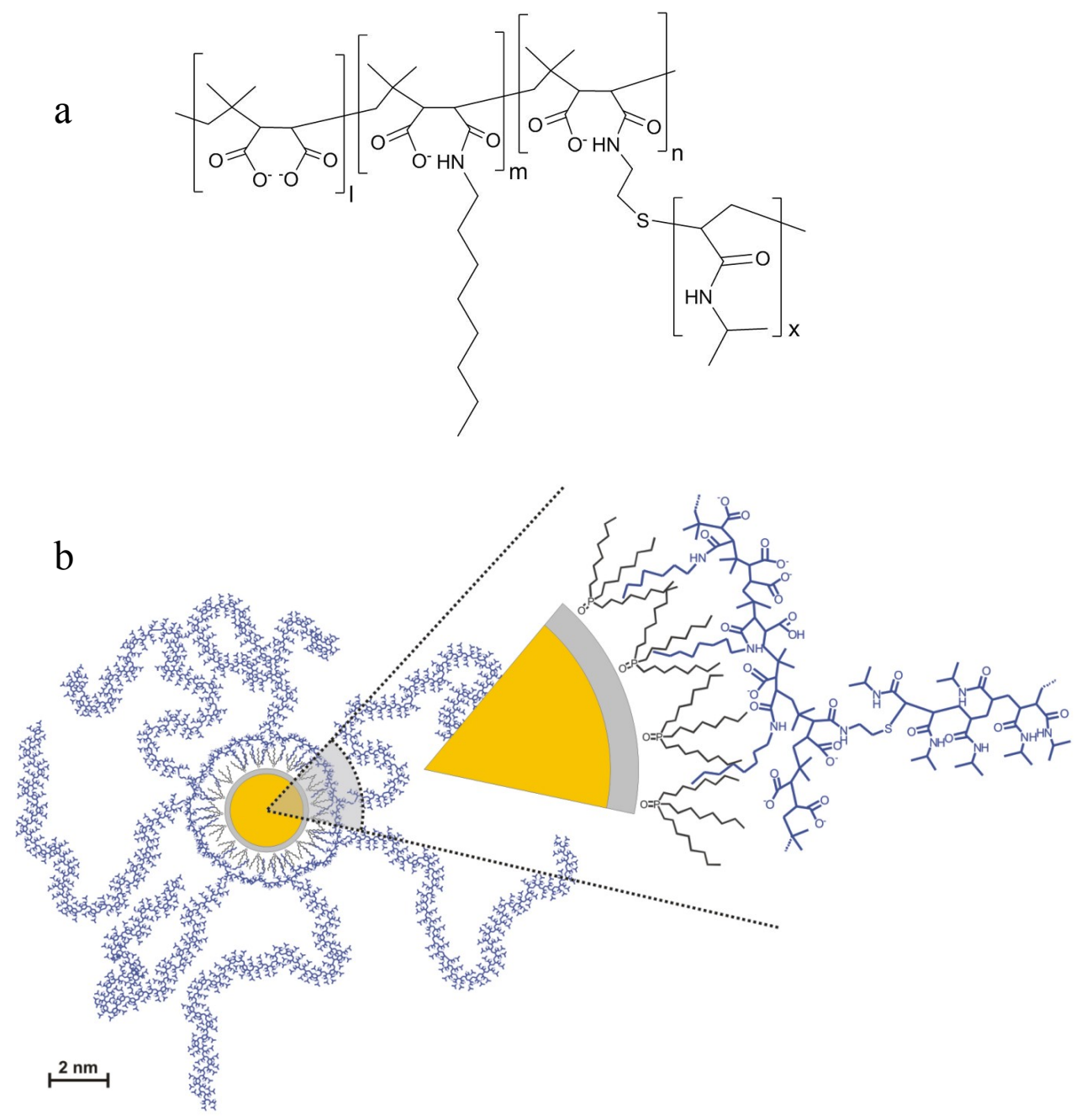

Figure 6.2. a) Schematic structure of the amphiphilic polymeric coatings after grafting of $\mathrm{NH}_{2}$-terminated PNIPAM and $n$-octylamine. $(1+\mathrm{m}+\mathrm{n}=38)$ to a poly(isobutylene-altmaleic anhydride) backbone. The number of monomer units in the PNIPAM chain is denoted with $\mathrm{x}$. b) Schematic representation of a TOPO-QD coated with the amphiphilic polymeric coating bearing PNIPAM side chains. The zoomed-in section shows details of the polymeric coating.

Upon completion of the reaction the amphiphilic polymers include octyl side groups for interaction with TOPO present on the QD surface, carboxyl groups for water dispersability and PNIPAM chains to induce temperature responsiveness. The compositions of polymers P1, P10, and P25 were evaluated by ${ }^{1} \mathrm{H}$ NMR and are presented in Table 6.1. 
Table 6.1. Composition of amphiphilic, PNIPAM-functionalized polymers used to transfer QDs into water.

\begin{tabular}{|c|c|c|c|c|c|c|}
\hline \multirow[b]{2}{*}{ Polymer } & \multirow{2}{*}{$\begin{array}{l}\mathrm{M}_{\mathrm{n}}^{\mathrm{a}} \times 10^{3} \\
{[\mathrm{~g} / \mathrm{mol}]}\end{array}$} & \multirow{2}{*}{$\begin{array}{l}\text { PNIPAM } \\
M_{\mathrm{w}} \times 10^{3} \\
{[\mathrm{~g} / \mathrm{mol}]}\end{array}$} & \multirow{2}{*}{$\begin{array}{l}\text { Indices } \\
1, \mathrm{~m}, \mathrm{n}, \mathrm{x}\end{array}$} & \multicolumn{3}{|c|}{$\%$ of functional groups } \\
\hline & & & & carboxylic & $\begin{array}{l}n \text {-octyl } \\
\text { amide }\end{array}$ & PNIPAM \\
\hline P1 & 28.7 & 1 & $0,19,19,8$ & 50 & 25 & 25 \\
\hline P10 & 41.9 & 10.8 & $16,19,3,95$ & 71 & 25 & 4 \\
\hline $\mathbf{P 2 5}$ & $19.8^{b}$ & 25.4 & $25,13,0.4,220$ & 82.7 & 16.7 & 0.6 \\
\hline
\end{tabular}

${ }^{\mathrm{a}}$ Number molar mass calculated from the NMR spectrum.

${ }^{\mathrm{b}}$ Average molar mass for a mixture of two polymers. ${ }^{\dagger}$

The number of PNIPAM chains per poly(isobutylene-alt-maleic anhydride) backbone was equal to 19, 3, and 0.4 for polymers P1, P10, and P25, respectively. All polymers displayed an LCST with a transition onset occurring at temperatures to within $1{ }^{\circ} \mathrm{C}$ equal to that of the PNIPAM (Figure 6.3). Therefore, grafting of the PNIPAM chains to the anhydride backbone did not modify their LCST behavior.

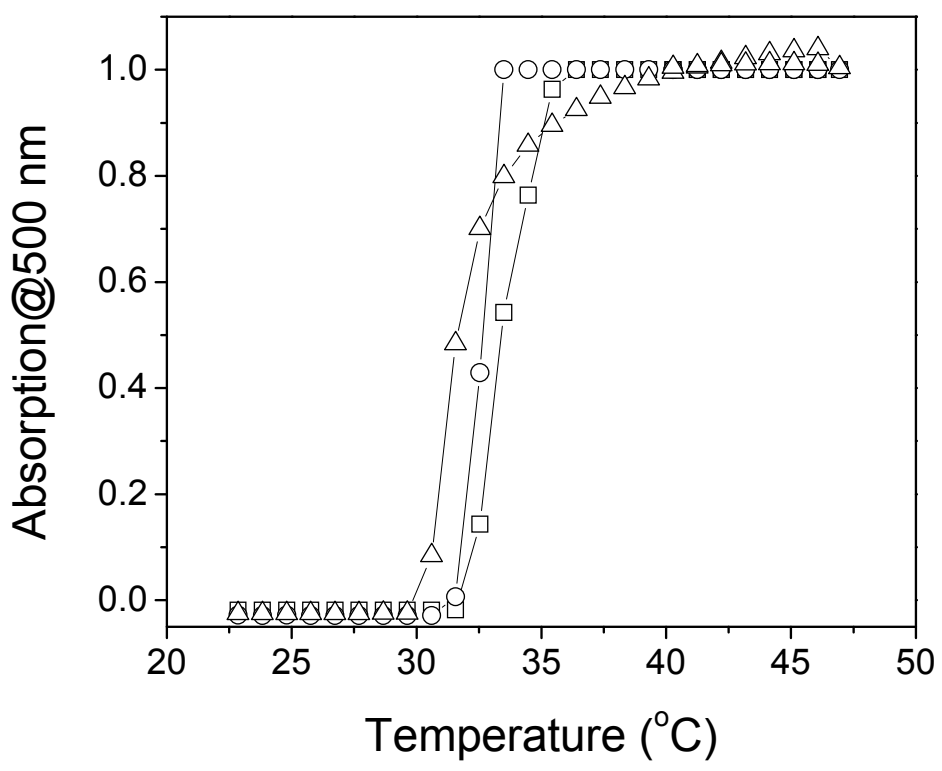

Figure 6.3. Normalized absorption of aqueous solutions of amphiphilic polymers P1 (口), P10 $(\circ)$ and P25 $(\Delta)$ measured at $500 \mathrm{~nm}$ as a function of temperature.

\footnotetext{
${ }^{\dagger}$ Considering the size of PNIPAM and $\mathrm{n}=0.4$ there are most likely two types of chains present, one without any PNIPAM side chain $\left(\mathrm{n}=0 \mathrm{M}_{\mathrm{w}}=9000 \mathrm{~g} / \mathrm{mol}\right)$ and the other with a single PNIPAM side chain $\left(\mathrm{n}=1, \mathrm{M}_{\mathrm{w}}=34000 \mathrm{~g} / \mathrm{mol}\right)$.
} 
Hydrophobic TOPO-coated QDs were transferred from organic solution to water using polymers P1, P10 and P25; as a result assemblies R1, R10 and R25 were formed. All polymers efficiently coated the QD surface rendering the QD/polymer assemblies hydrophilic.

The absorption and emission spectra of QDs R1, R10, and R25 coated with the polymers P1, P10 and P25, respectively, are shown in Figure 6.4. The position of the first absorption peak and that of the emission peak was not altered significantly by the presence of the polymeric coating. However, the emission intensity clearly decreased in comparison with TOPO-coated QDs of the same concentration (data not shown). Although such a decrease in intensity was reported previously for hydrophilic, polymer-coated QDs [34], we cannot rule out that other factors, including multiple particle aggregation play here a role. Once transferred to water the PNIPAM-coated QDs displayed colloidal stability for a period of several months with no visible precipitation. The stability of the polymer coating covering the QD surface is a result of hydrophobic-hydrophobic interactions between the octylamine groups of the polymer with the TOPO layer at the QD surface. The colloidal stability of QD/PNIPAM assemblies in water is provided by the hydrophilic nature of PNIPAM and the carboxyl groups on the polymer backbone.

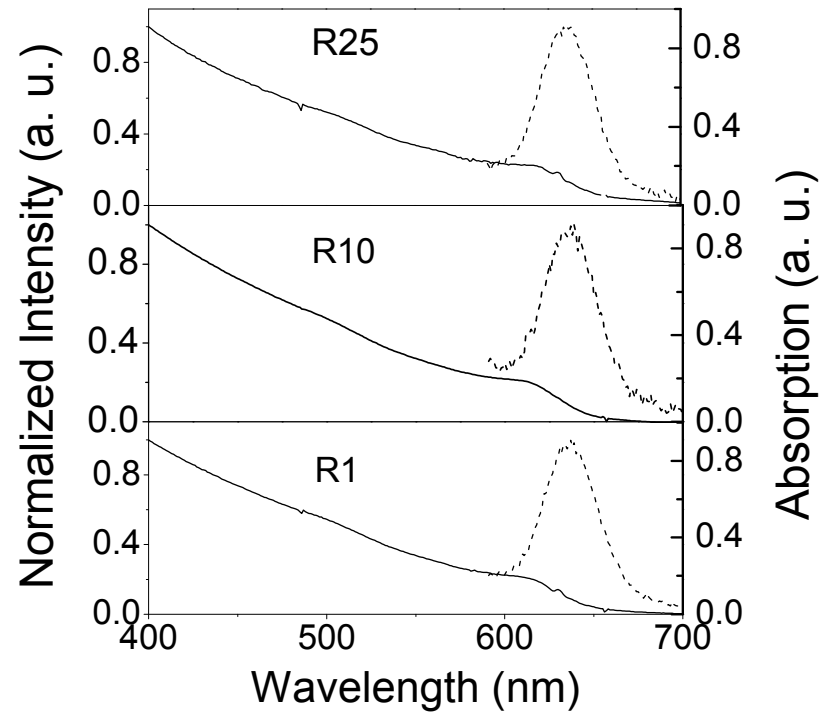

Figure 6.4. Normalized emission (dash) and absorption (solid) spectra of water solutions of CdSe/ZnS QDs R1, R10 and R25 coated with polymers P1, P10 and P25, respectively. 
In order to characterize the temperature response of QDs coated with the new amphiphilic coatings, a series of temperature-dependent absorption and emission measurements was performed (Figure 6.5). Upon reaching temperatures above the LCST, the absorbance of samples R1, R10 and R25 increased. A sharp transition at $32{ }^{\circ} \mathrm{C}$ was observed for sample R25. The transitions for samples R10 and R1 occurred at $34{ }^{\circ} \mathrm{C}$ and $33{ }^{\circ} \mathrm{C}$, respectively.

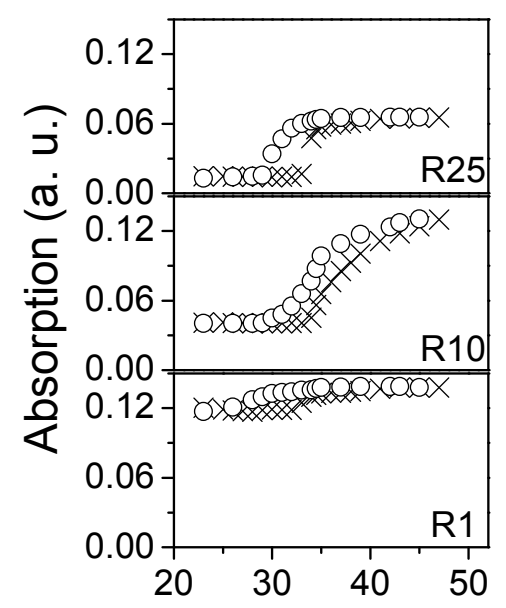

Temperature $\left({ }^{\circ} \mathrm{C}\right)$

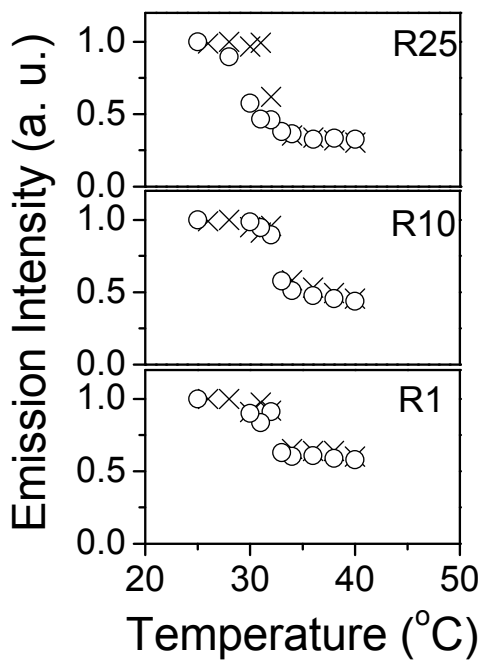

Figure 6.5. Absorption at $500 \mathrm{~nm}$ (left), and luminescence emission at $640 \mathrm{~nm}$ (right) recorded during a heating $(\mathrm{X})$ and subsequent cooling $(\mathrm{O})$ cycle. For comparison, the initial measurements were normalized to 1.0 on the emission plots.

Interestingly, the QD luminescence emission intensity was also influenced by temperature. Pronounced transitions in the luminescence intensity for all polymer-coated QD samples, as shown in Figure 6.5, occurred to within the experimental error at the same temperatures as the transitions visible in the absorbance. This suggests that the LCST behavior of PNIPAM was responsible for the changes in the luminescence emission of the QDs. We performed a control experiment, where we heated the same QDs coated with a PEG-modified amphiphilic polymer [35] instead of PNIPAM. No transition was observed within the probed temperature range, and the changes in the emission intensity followed a similar trend as reported in the literature [5]. This indicates that changes within the PNIPAM shell were responsible for the observed behavior. 
The transitions in absorption and luminescence associated with the LCST of PNIPAM were clearly reversible (Figure 6.5). The absorption and the luminescence intensities recovered back to the initial values when the temperature was decreased below the LCST. Similar trends in the emission and absorption, including the extent of the hysteretic behavior was observed for each of the samples, giving additional evidence that PNIPAM is responsible for the observed changes. The reversibility of the LCST-induced transitions was also studied by heating above LCST to $50{ }^{\circ} \mathrm{C}$ and cooling down to $25^{\circ} \mathrm{C}$ over multiple heating/cooling cycles (Figure 6.6). To compare the influence of temperature on the absorption and emission intensities for different samples, the initial values were normalized to 0.1 for absorption and to 1.0 for emission.
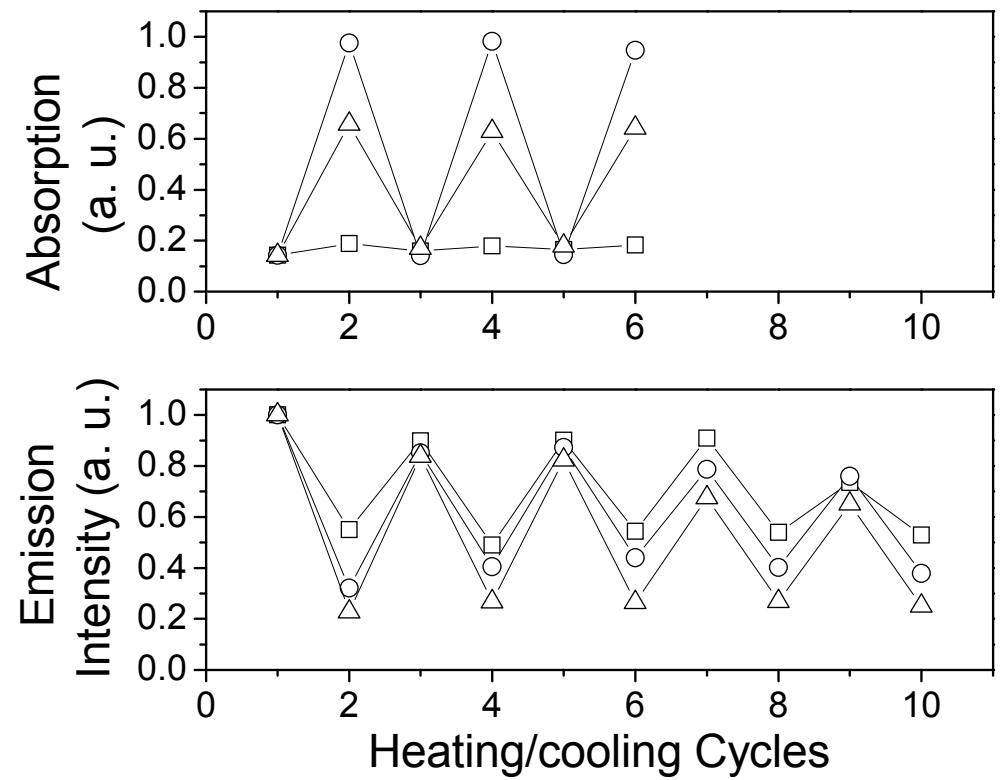

Figure 6.6. Optical absorption at $500 \mathrm{~nm}$ and emission intensity at $\lambda=640 \mathrm{~nm}$ of R1 (口), $\mathbf{R 1 0}(\circ)$ and $\mathbf{R 2 5}(\Delta)$ recorded at $25^{\circ} \mathrm{C}$ (odd numbers) and at $50{ }^{\circ} \mathrm{C}$ (even numbers) in water.

Different response to changes in temperature depending on the polymer coating is a result of a convolution of different factors including the molar mass of the grafted PNIPAM chains to the anhydride backbone, its grafting density, and the number of the PNIPAM chains on the QD surface. Unfortunately we are unable to separate each of these factors. For our system, it is notoriously difficult to estimate the number of polymer chains in the polymeric coating on the QD surface. Additionally, we do not rule out that larger assemblies are formed in water, especially for coatings with longer PNIPAM chains. 
Changes in the morphology of the samples upon crossing the LCST of PNIPAM were followed by temperature-dependent light scattering experiments (Figure 6.7). Upon heating to $50{ }^{\circ} \mathrm{C}$, the hydrodynamic radius decreased. A transition was clearly observed at temperatures slightly higher than the onset temperatures of the transitions observed in absorption and luminescence experiments, however still within the transitions widths.

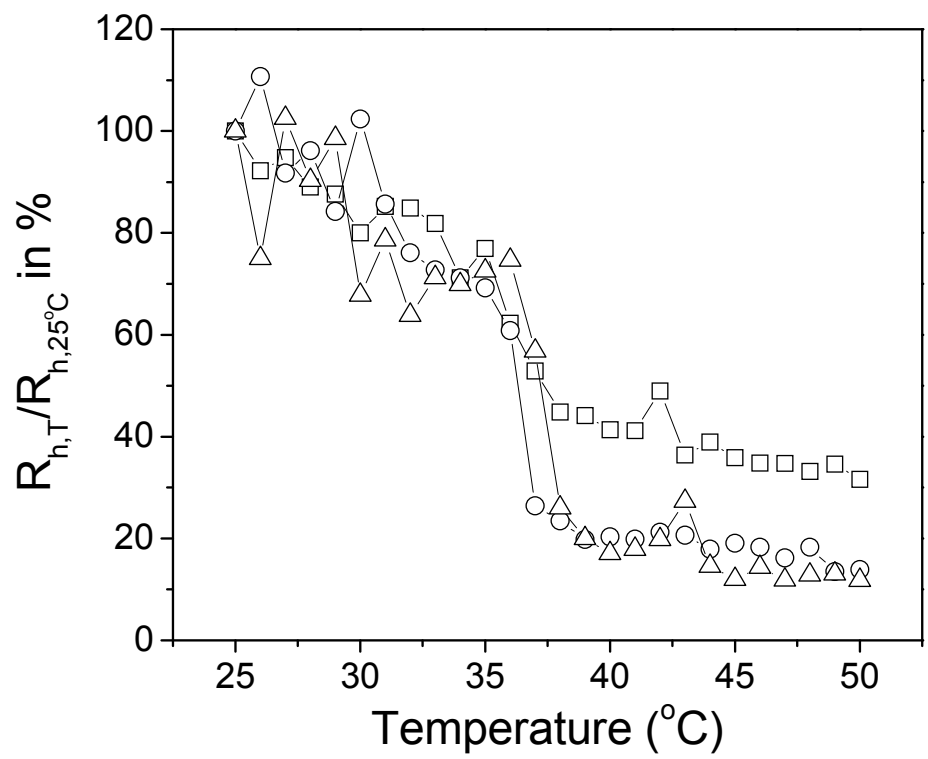

Figure 6.7. Effective hydrodynamic radius $\left(\mathrm{R}_{\mathrm{h}, \mathrm{T}}\right)$ of PNIPAM-coated QDs R1 ( $\left.\square\right)$, R10 (O) and $\mathbf{R 2 5}(\Delta)$ as a function of temperature normalized to the hydrodynamic radius at $25{ }^{\circ} \mathrm{C}$

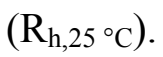

Temperature dependent electrophoretic mobility experiments shown in Figure 6.8 revealed that regardless of the polymeric coating used, the samples displayed low electrophoretic mobility at $\mathrm{T}<\mathrm{LCST}$. The particle mobility was observed to increase sharply as the temperature was raised above the LCST, with a transition onset at temperatures similar to those observed in light scattering experiments. The high electrophoretic mobility above LCST was attributed to the presence of appreciable amount of carboxylic groups on the QD surface. Similar results were obtained by Liu et al. for PNIPAM-coated silica particles [23]. They explained their results by shifts of the shear plane away and closer to the particle surface depending on the morphology of the PNIPAM chains. Exposure of highly hydrophilic carboxylate groups prevents the QDs forming large aggregates [40], what is consistent with the observed decrease of the size of the assemblies upon heating. This is in contrast with previous studies of the LCST behavior of nanoparticles coated with thermoresponsive 
polymers where the collapse of the polymer chains on the surface of the nanoparticles leads to aggregation $[4,28-30]$.

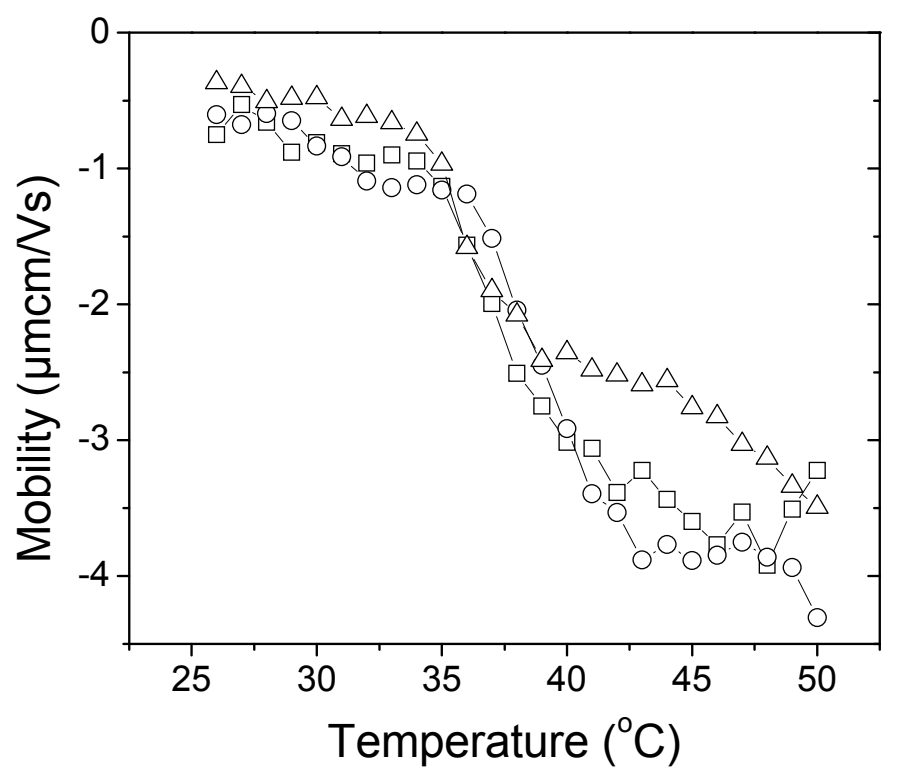

Figure 6.8. Electrophoretic mobility of samples R1 ( $\square)$, R10 (०) and R25 ( $\Delta$ ) measured as a function of temperature.

The above-presented results suggest a complicated process during the LCST transition involving PNIPAM chain collapse and modification of inter-dot interactions resulting in a decrease of the assemblies' size. More studies on the morphology of the samples, which is beyond the scope of this thesis, are needed to elucidate the exact mechanism for the changes in the luminescence intensity. Regardless, it is important to note that our approach offered an easy QD functionalization protocol based on amphiphilic polymeric coatings, and that the PNIPAM-coated QDs display temperature induced changes in the emission. Additionally the presence of the carboxylate groups on the surace of the QD/polymer assembly prevented aggregation of the QDs above LCST. This may prove useful in context of QD applications in drug delivery and sensing.

\subsection{Conclusions}

An efficient method for the fabrication of water soluble, temperature-responsive QD/PNIPAM assemblies was presented. A maleic anhydride-based polymer was used as backbone for grafting PNIPAM chains of three different molar masses. The QDs coated with the polymers were successfully transferred to water. The hybrid nanoparticles exhibited 
LCST behavior at temperature similar to those of free polymers. These nano-sized, temperature responsive hybrid particles hold a great promise towards realization of sensor devices with a broad range of applications.

\subsection{References}

1. $\quad$ Zhang, J.L., Srivastava, R.S.; Misra, R.D.K., Langmuir 2007. 23: p. 6342.

2. Hoffman, A.S. and Stayton, P.S. Prog Polym Sci, 2007. 32: p. 922.

3. Narain, R.; Gonzales, M.; Hoffman, A.S.; Stayton, P.S.; Krishnan, K.M. Langmuir, 2007. 23(11): p. 6299-304.

4. Lai, J.J.; Hoffman, J.M.; Ebara, M.; Hoffman, A.S.; Estournes, C.; Wattiaux, A.; Stayton, P.S., Langmuir, 2007. 23(13): p. 7385-91.

5. Tagit, O.; Tomczak, N.; Benetti, E.M.; Cesa, Y.; Blum, C.; Subramaniam, V.; Herek, J.L.; Vancso, G.J., Nanotechnology, 2009. 20: p. 185501.

6. Wang, S.; Song, H.; Ong, W.Y.; Han, M.Y.; Huang, D., Nanotechnology, 2009. 20: p. 425102.

7. Ionov, L.; Sapra, S.; Synytska, A.; Rogach, A.L.; Stamm, M.; Diez, S., Adv Mater, 2006. 18(11): p. 1453.

8. Schild, H.G., Prog Polym Sci, 1992. 17(2): p. 163.

9. $\quad$ Schmaljohann, D., Adv Drug Deliv Rev, 2006. 58: p. 1655.

10. Wu, K.; Shi, L.; Zhang, W.; An, Y.; Zhu, X.X., J App Polym Sci, 2006. 102: p. 3144.

11. Deng, Y.H.; Yang, W.L.; Wang, C.C.; Fu, S.K., Adv Mater, 2003. 15: p. 1729.

12. Zhang, S.; Zhang, L.; He, B.; Wu, Z., Nanotechnology, 2008. 19: p. 325608.

13. He, J.; Chen, J.-Y.; Wang, P.; Wang, P.-N.; Guo, J.; Yang, W.-L.; Wang, C.-C.; Peng, Q., Nanotechnology, 2007. 18(41): p. 415101.

14. Cheng, Z.; Liu, S.; Gao, H.; Tremel, W.; Ding, N.; Liu, R.; Beines, P.W.; Knoll, W., Macromol Chem Phys, 2008. 209: p. 1145.

15. Zhu, M.Q.; Wang, L.Q.; Exarhos, G.J.; Li, A.D., J Am Chem Soc, 2004. 126(9): p. 2656.

16. Raula, J.; Shan, J.; Nuopponen, M.; Niskanen, A.; Jiang, H.; Kauppinen, E.I.; Tenhu, H., Langmuir, 2003. 19: p. 3499.

17. Shan, J.; Nuopponen, M.; Jiang, H.; Kauppinen, E,; Tenhu, E., Macromolecules, 2003. 36: p. 4526.

18. Li, D.; He, Q.; Cui, Y.; Wang, K.; Zhang, X.; Li, J,.Chem A Eur J, 2007. 13(8): p. 2224. 
19. Shan, J.; Nuopponen, M.; Jiang, H.; Viitala, T.; Kauppinen, E.; Kontturi, K.; Tenhu, H., Macromolecules, 2005. 38: p. 2918.

20. Shan, J.; Zhao, Y,; Granqvist, N.; Tenhu, H., Macromolecules, 2009. 42: p. 2696.

21. Salmaso, S.; Caliceti, P.; Amendola, V.; Meneghetti, M.; Magnusson, J.P.; Pasparakis, G.; Alexander, C., J Mater Chem, 2009. 19: p. 1608.

22. Kim, D.J.; Kang, S.M.; Kong, B.; Kim, W.-J.; Paik, H.-J.; Choi, H.; Choi, I.S., Macromol Chem Phys, 2005. 206: p. 1941.

23. Liu, J.; Pelton, R.; Hrymak, A.N., J Colloid Interface Sci, 2000. 227(2): p. 408.

24. Li, D.; Jones, G.L.; Dunlap, J.R.; Hua, F.; Zhao, B., Langmuir, 2006. 22(7): p. 3344.

25. Karg, M.; Pastoriza-Santos, I.; Liz-Marzan, L.M.; Hellweg, T., Chem Phys Chem, 2006. 7(11): p. 2298-301.

26. Bruchez, M., Jr.; Moronne, M.; Gin, P.; Weiss, S.; Alivisatos, A.P., Science, 1998. 281(5385): p. 2013-6.

27. a) Tomczak, N.; Janczewski, D.; Han, M.-Y.; Vancso, G.J., Prog Polym Sci, 2009. 34: p. 393. b) Janczewski, D.; Tomczak, N.; Han, M.-Y.; Vancso, G.J., Macromolecules, 2009. 42: p. 1801.

28. Hou, Y.; Ye, J.; Gui, Z.; Zhang, G., Langmuir, 2008. 24: p. 9682.

29. Ye, J.; Hou, Y.; Zhang, G.; Wu, C., Langmuir, 2008. 24: p. 2727.

30. Zhou, L.; Gao, C.; Xu, W., J Mater Chem, 2009. 19: p. 5655.

31. Shiraishi, Y.; Adachi, K.; Tanaka, S.; Hirai, T., J Photochem Photobiol A: Chem, 2009. 205: p. 51.

32. Cheng, Z.; Liu, S.; Beines, P.W.; Ding, N.; Jakubowicz, P.; Knoll, W., Chem Mater, 2008. 20: p. 7215.

33. Yu, W.W.; Chang, E.; Falkner, J. C.; Zhang, J.; Al-Somali, A. M.; Sayes, C. M.; Johns, J.; Drezek, R.; Colvin, V. L., J Am Chem Soc, 2007. 129(10): p. 2871.

34. Pellegrino, T.; Manna, L.; Kudera, S.; Liedl, T.; Koktysh, D.; Rogach, A.L.; Keller, S.; Rädler, J.; Natile, G.; Parak, W.J. H, Nano Lett, 2004. 4(4): p. 703.

35. Jańczewski, D.; Tomczak, N.; Khin, Y.W.; Han, M.Y.; Vancso, G.J., Eur Poly J, 2009. 45(1): p. 3.

36. Berne, B.J.; Pecora, R. Dynamic Light Scattering with Applications to Chemistry, Biology and Physics, Wiley, New York, 1976.

37. Van Blaaderen, A.; Vrij, A., Langmuir 1992. 8(12): p.2921

38. Pons, T.; Uyeda, H.T.; Medintz, I.L.; Mattoussi, H., J. Phys.Chem.B. 2006, 110(41): p. 20308 
39. Yu, W.W.; Qu, L.; Guo, W.; Peng, X., Chem Mater, 2003. 15(14): p. 2854

40. Hou, Y.; Ye, J.; Wei, X.; Zhang, G., J Phys Chem B, 2009. 113: p. 7457 



\section{Chapter 7}

\section{Optical characterization of thermo-responsive polymer- quantum dot nanoparticles}

Structural and optical characterization of water soluble, thermo-responsive quantum dot/ poly(N-isopropyl acrylamide) (QD/PNIPAM) hybrid particles using fluorescence correlation spectroscopy (FCS) and time correlated single photon counting (TCSPC) measurements performed at temperatures below and above the lower critical solution temperature (LCST) of PNIPAM is reported. By increasing the temperature above the LCST, the collapse of the PNIPAM chains covering the QDs is revealed by FCS. Despite the significant structural change, the TCSPC measurements show that fluorescence lifetimes remain on the same order of magnitude at T>LCST. Such QD/PNIPAM hybrid particles with water solubility and robust thermo-responsive behavior at physiologically relevant temperatures will prove useful for in vivo applications. 



\subsection{Introduction}

The unique optical properties of quantum dots (QDs), such as broad absorption spectrum and narrow, size-tunable emission peaks [1, 2], make them attractive for many applications in life sciences and optoelectronics [3, 4]. Stimuli-responsive polymers have received great interest due to their physical or chemical changes in response to changes in environmental conditions such as temperature, $\mathrm{pH}$, ionic strength, and electromagnetic radiation [5]. Poly( $N$-isopropyl acrylamide) (PNIPAM) is a well-known example of a thermoresponsive polymer, which undergoes a sharp coil-globule transition in water at its lower critical solution temperature (LCST, $\sim 32{ }^{\circ} \mathrm{C}$ ). The polymer changes from a hydrophilic state below the LCST into a hydrophobic state above the critical temperature [6]. Fortuitously, the phase transition of PNIPAM occurs in the physiological temperature range, making the polymer especially promising for biological applications. Recently, integration of PNIPAM with QDs has been pursued in an attempt to create smart, nanosized hybrid luminescent materials [7-12]. The synthesis of such materials is discussed in Chapter 6. The optical characterization of QDs and QD/polymer assemblies as a function of temperature has been limited to simple absorption and emission measurements [13], and the colloidal properties, such as particle size distribution and aggregation behavior have been monitored mostly by light scattering and TEM measurements [14]. Conventional light scattering measurements of QDs lack single molecule sensitivity, and bulk measurements can be influenced by aggregation of the nanoparticles [15]. TEM measurements give accurate and quantitative size values; however, it is not possible to study the materials in solution and at high temperatures with this technique. Therefore, it is necessary to use complementary techniques to determine the colloidal and optical properties of temperature-responsive QD/polymer hybrid assemblies [16] at different temperatures.

Fluorescence correlation spectroscopy (FCS) is a sensitive optical detection method with a wide range of applications [17-21]. FCS is based on the measurement of the fluorescence fluctuations as a light emitter passes through the confocal volume. In contrast to photon correlation spectroscopy based on dynamic light scattering, which is also used for characterization of diffusing molecules, FCS provides information about the photophysical properties of the fluorescent particles, such as photon count rates. FCS is therefore a particularly attractive method for studying the optical and colloidal properties of engineered QDs for situations, where the surface coating affects both the colloidal and photophysical stabilities. In addition, detection of fluorescence rather than scattered light makes FCS a more sensitive and specific technique for luminescent particles. 
The theory of FCS has been described elsewhere [19, 21, 22]. Briefly, the observed fluorescence intensity fluctuations are translated into the autocorrelation function shown in Eq. (7.1):

$$
g(\tau)=\langle\delta I(t) \delta I(t+\tau)\rangle+\langle I(t)\rangle^{2}
$$

The fluorescence fluctuation around the average intensity, $\delta I(t)$, is monitored and correlated to the intensity obtained at time $t+\tau$ to generate the autocorrelation function. Normalization of the autocorrelation function yields Eq. (7.2.):

$$
G(\tau)=1+\frac{\langle\delta I(t) \delta I(t+\tau)\rangle}{\langle I(t)\rangle^{2}}
$$

The normalized autocorrelation function of the fluorescence fluctuations resulting from the diffusion of the particles through the confocal volume is used to obtain information about the number of particles per detection volume and the characteristic timescale of diffusion, by equating Eq. (7.2) to the autocorrelation function obtained for a 3D Gaussian volume shown in Eq. (7.3):

$$
G(\tau)=1+\frac{1}{N}\left(1+\frac{\tau}{\tau_{D}}\right)^{-1}\left(1+S^{2} \frac{\tau}{\tau_{D}}\right)^{-\frac{1}{2}}
$$

where, $N$ represents the number of fluorophores in the confocal volume at any time, $\tau_{D}$ is the diffusion time of the molecule through the confocal volume, and $S$ is the structure parameter. $\mathrm{S}$ is equal to the ratio of axial $\left(\omega_{z}\right)$ and radial $\left(\omega_{r}\right)$ distances at which the intensity of the Gaussian excitation falls to $\mathrm{e}^{-2}$ of its maximum [14]. The inverse relation between the autocorrelation signal and the number of fluorophores enables measurements in the nanomolar range.

FCS has been recently employed to determine the diffusion behavior of fluorescent nanoparticles [14, 23, 24], including Si nanoparticles and QDs [15, 25, 26], and the optical properties of fluorescent nanoparticles [27]. Since polymer coatings influence both the colloidal stability and the emission properties of the QDs, FCS turns out to be a very 
attractive characterization method for QDs coated with thermo-responsive polymers as a function of temperature.

A complementary technique, TCSPC, allows measurements of fluorescence decay profiles [28]. TCSPC has been widely used for the characterization of colloidal semiconductor nanoparticles [29], organic fluorophores [30], and molecules labeled with fluorescent moieties [31] with picosecond time resolution down to the single molecule level [32]. The pertaining details of both methods can be found in Chapter 3.

In this chapter, we report a detailed optical characterization study of QD/PNIPAM hybrid nanoparticles fabricated using an amphiphilic coating (synthesis of these particles is discussed in Chapter 6) at temperatures below and above the LCST of PNIPAM, using fluorescence correlation spectroscopy (FCS) and time-correlated single photon counting (TCSPC). These techniques can give novel insights into the colloidal and optical properties of the investigated systems.

\subsection{Experimental section}

The synthesis of CdSe/ZnS core/shell QDs and grafting of poly(isobutylene-altmaleic anhydride $)\left(\mathrm{M}_{\mathrm{n}}=6000 \mathrm{~g} / \mathrm{mol}\right)$ with n-octylamine and amino-terminated PNIPAM $\left(\mathrm{NH}_{2}\right.$-PNIPAM, with three different molar masses $\left(\mathrm{M}_{\mathrm{n}}\right) 1000 \mathrm{~g} / \mathrm{mol}, \mathrm{P} 1 ; 10800 \mathrm{~g} / \mathrm{mol}, \mathrm{P} 10$; and $25400 \mathrm{~g} / \mathrm{mol}, \mathrm{P} 25$ ) was described in Chapter 6 . The aqueous solutions of polymer-coated QDs R1, R10 and R25 were filtered through a $0.22 \mu \mathrm{m}$ MILEX PES membrane filter and centrifuged at $10000 \mathrm{rpm}$ for 30 minutes prior to measurements.

The polymers P1, P10 and P25 used for coating the QDs had different PNIPAM content, of $19 \%, 3 \%$, and $0.4 \%$, respectively, as determined by ${ }^{1} \mathrm{H}$ NMR. This means that in addition to different molar mass of the PNIPAM chains, the grafting density of the PNIPAM chains was also different. The details of the temperature-dependent steady-state absorption and emission measurements, and light scattering experiments (DLS) were described in Chapter 6.

Fluorescence correlation spectroscopy (FCS) measurements were performed using a ConfoCor2/LSM510 microscope (Carl Zeiss, Germany). Excitation light was provided by an Ar ion laser (458, 488 and $514 \mathrm{~nm}$ ) and two He-Ne lasers (543 and $633 \mathrm{~nm})$. The ConfoCor2 microscope contained a set of appropriate excitation, dichroic and emission filters. The excitation laser power was set at approximately $2 \mu \mathrm{W}$. Light was focused onto the sample and the luminescence was collected using a water immersion C-Apochromat 40x objective lens with a numerical aperture of 1.2 (Carl Zeiss, Germany), and detected using two fiber coupled 
avalanche photodiodes. AIM software (Zeiss-EMBL) was used for the autocorrelation analysis. Data analysis was performed on a PC workstation equipped with either the AIM software or with an FCS Data Processor 1.4 (Scientific Software Technologies Software Centre, Blarus), which allows global fitting using an autocorrelation function describing three-dimensional diffusion with triplet state kinetics. The volume of the confocal spot was determined using a solution of $50 \mathrm{nM}$ Rhodamine 110 with known diffusion coefficient $(\mathrm{D}=$ $\left.420 \mu \mathrm{m}^{2} / \mathrm{s}\right)$.

Time-resolved fluorescence data were obtained at an emission wavelength of $640 \mathrm{~nm}$ by using a HORIBA Jobin Yvon FluoroMax-4 equipped with a NanoLED pulsed laser diode excitation source from IBH $(F W H M=1 \mathrm{~ns}, 462 \mathrm{~nm})$ and a TCSPC detection system (FluoroHub, HORIBA Jobin Yvon) based on time to amplitude conversion (TAC). The fluorescence time-resolved data were fitted by using the DAS6 Decay Analysis Software package from HORIBA Jobin Yvon. An instrument response function was recorded on strongly scattering particles (Ludox) in water by using identical settings as when measuring the samples; only the emission wavelength was adjusted. Deconvolution analysis of the luminescence decay with 3 exponentials was carried out. The data were recorded at $20{ }^{\circ} \mathrm{C}$ and at $55^{\circ} \mathrm{C}$.

\subsection{Results and discussion}

The QDs exhibited a broad absorption spectrum with a first absorption peak at $\sim 620$ $\mathrm{nm}$ and a narrow emission spectrum (35 nm full width at half maximum) located at $638 \mathrm{~nm}$. Coating the QDs with the polymer did not induce any noticeable changes in the positions of the absorption and emission peaks; however, the fluorescence intensity was observed to decrease compared to that of the bare QDs in chloroform at the same concentration (see Chapter 6).

Fluorescence correlation spectroscopy (FCS) at temperatures below and above the LCST of PNIPAM was performed in order to determine the colloidal properties of QD/PNIPAM hybrid particles using the fluctuations in their luminescence intensities. A representative raw data set, showing the intensity time-traces and corresponding correlation curves for QDs coated with thermo-responsive PNIPAM at temperatures below $\left(20{ }^{\circ} \mathrm{C}\right)$ and above $\left(55^{\circ} \mathrm{C}\right)$ the LCST is shown in Figure 7.1. Comparison of the raw data for each sample (only R10 is shown here) obtained at T<LCST and T $>$ LCST show distinct changes in the shape and amplitude of the correlation curves. 


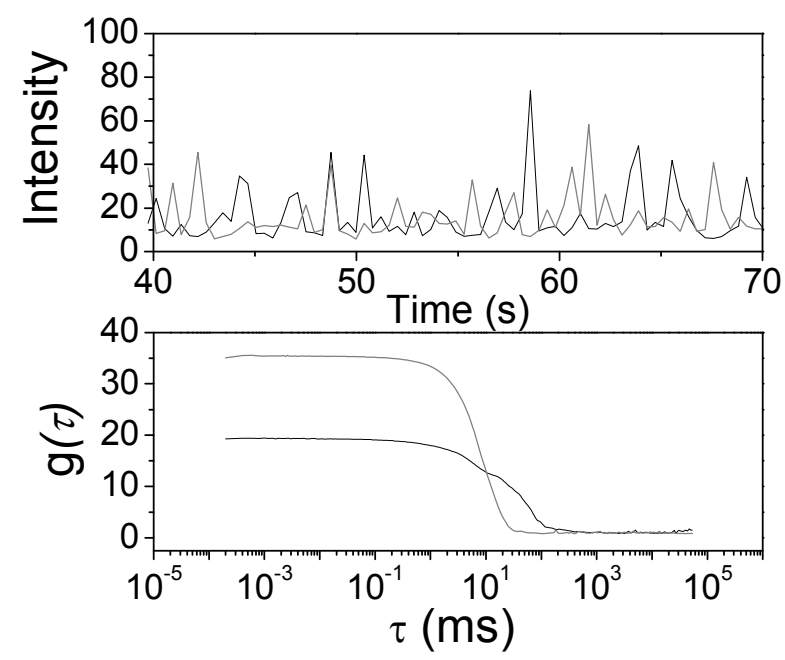

Figure 7.1. Raw correlation curves and intensity time-traces of sample R10 measured at $\mathrm{T}<$ LCST (black) and T $>$ LCST (gray).

Crossing the LCST resulted in higher amplitudes and steeper slopes of the correlation curves. The observed increase in the amplitudes of the correlation curves at T>LCST can be due to decrease in the number of luminescent species in the solution with unchanged luminescence intensity or to decrease of the luminescence intensity with unchanged particle number, or both. Dong et al. previously reported that the amplitudes of the FCS curves for CdTe QDs solutions increase upon addition of a quencher [27]. The increase of the amplitudes was explained by a decrease in the number of bright QDs present in the solution, causing the apparent number of molecules in the confocal volume to decrease, giving rise to higher $g$ amplitudes. In another study, the dependence of the shape of the FCS autocorrelation curves on the excitation laser power was presented [33]. At higher intensities the autocorrelation intensity $\left(g_{0}\right)$ drops due to excitation saturation [34]. In our measurements the excitation laser power was kept constant to compare the effects of the different coatings, and to disentangle the intensity-dependence of the FCS curve. The potential artifacts that would be introduced due to heating were eliminated by considering the temperature-induced changes in the physical parameters, such as viscosity of water in our calculations. Therefore, it is very likely that temperature-induced changes in the correlation curves are solely due to changes in the properties of QD/PNIPAM hybrids. As reported in Chapter 6, the steady-state measurements at T $>$ LCST showed that the luminescence intensity of the hybrid particles decrease at T $>$ LCST. Such decrease in the luminescence intensity at T $>$ LCST is most likely 
the reason of the higher amplitudes observed in the FCS autocorrelation curves upon crossing the LCST.

The $G(\tau)$ function used to obtain the diffusion times were averaged over 5 measurements, and normalized to $\mathrm{G}(0)=1$ for each sample. As a control, the autocorrelation curve of Rh110 was measured in parallel (Figure 7.2).

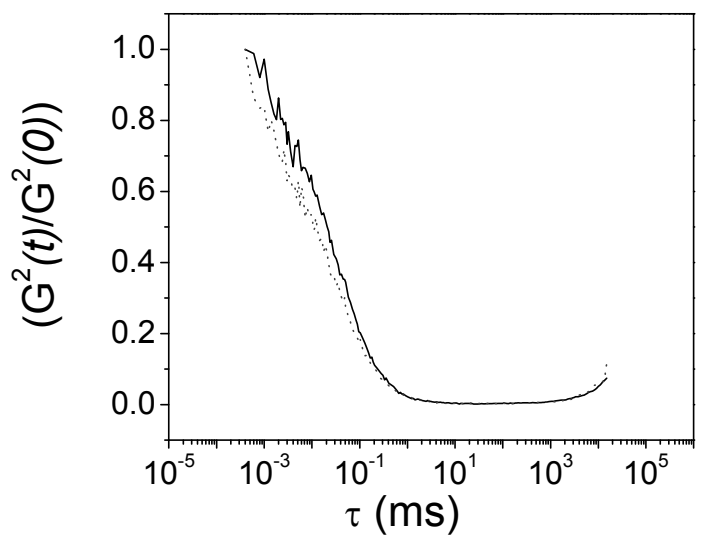

Figure 7.2. Normalized correlation functions of Rh1 10 measured at $\mathrm{T}<\mathrm{LCST}\left(20{ }^{\circ} \mathrm{C}\right.$, solid) and $\mathrm{T}>\operatorname{LCST}\left(55^{\circ} \mathrm{C}\right.$, dotted).

The correlation curves obtained at $\mathrm{T}>\mathrm{LCST}$ had steeper slopes when compared to those obtained at $\mathrm{T}<\mathrm{LCST}$ (Figure 7.3 left and right panels). It has previously been shown that the width of the correlation functions increase with the increasing molecule size [27], i.e., the characteristic diffusion times of the molecules are positively correlated with their size.
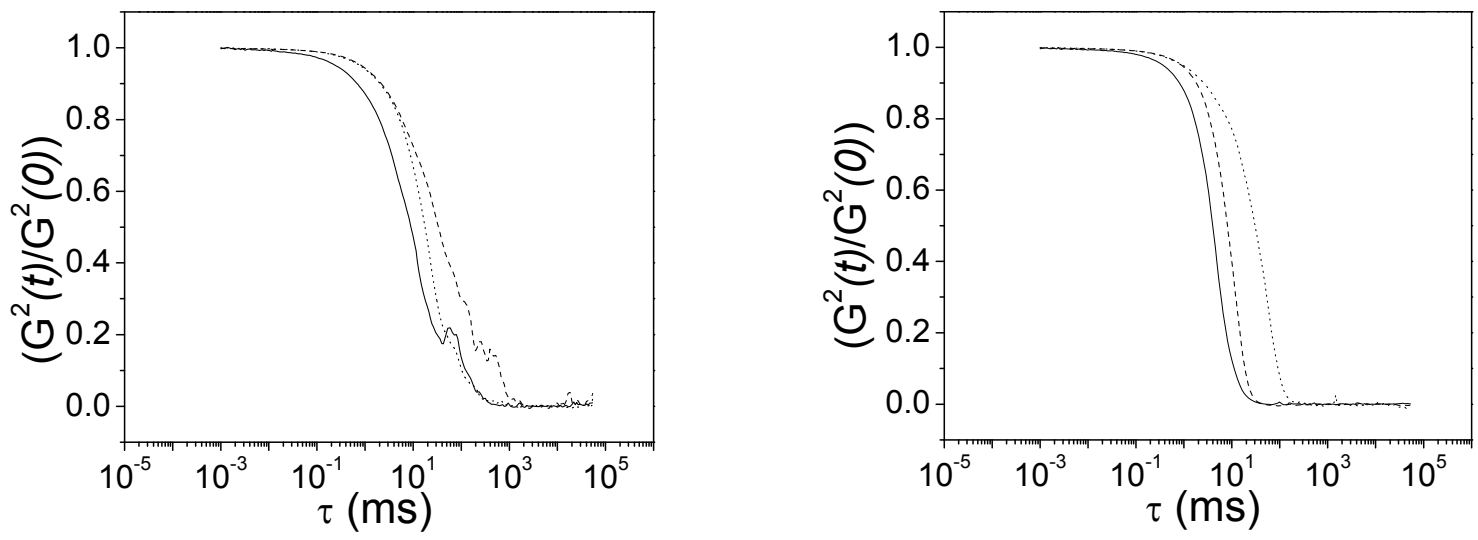

Figure 7.3. Normalized correlation functions of R1 (solid), R10 (dash) and R25 (dot) measured at $\mathrm{T}<\operatorname{LCST}\left(20^{\circ} \mathrm{C}\right.$, left $)$ and $\mathrm{T}>\operatorname{LCST}\left(55^{\circ} \mathrm{C}\right.$ right $)$. 
The diffusion times of the samples were determined using the slopes of the autocorrelation curves. At T $>$ LCST, the hybrid particles were observed to diffuse faster through the confocal volume, as indicated by the sharper decay in the correlation functions. In addition, the diffusion times of the nanoparticles increased as a function of PNIPAM chain-size grafted to the QD coatings.

The diffusion coefficients of the samples at temperatures below and above the LCST of PNIPAM were calculated using the Eq. (7.4):

$$
\tau_{D}=\frac{\omega_{0}^{2}}{4 D}
$$

where $\tau_{D}$ is the diffusion time of the particles through the confocal volume, $\omega_{0}$ is the radius of the confocal volume, and $D$ is the diffusion coefficient. $\omega_{0}$ was calculated by inserting the $\tau_{D}$ obtained for Rhodamine 110 into Eq. 7.4.

The diffusion coefficients obtained for each sample at temperatures below $\left(20^{\circ} \mathrm{C}\right)$ and above $\left(55^{\circ} \mathrm{C}\right)$ the LCST of PNIPAM are compared in Table 7.1.

Table 7.1. Diffusion coefficients of R1, R10 and R 25 at $\mathrm{T}<\mathrm{LCST}$ and $\mathrm{T}>\mathrm{LCST}$ as calculated from FCS correlation curves and light scattering measurements (DLS).

\begin{tabular}{c|c|c|c|c|c|c}
\cline { 2 - 6 } & \multicolumn{5}{c}{ Diffusion Coefficient D $\left(\times 10^{-12} \mathrm{~m}^{2} / \mathrm{s}\right)$} \\
\cline { 2 - 7 } & \multicolumn{3}{c}{ FCS } & R1 & R10 & R25 \\
\cline { 2 - 7 } & R1 & R10 & R25 & R1 & 0.05 & 0.3 \\
\hline T $<$ LCST & 1.4 & 0.3 & 0.98 & 0.7 & 0.7 & 4.4 \\
\hline T $>$ LCST & 7.7 & 4.9 & 1.00 & 3.6 & 0.7 \\
\hline
\end{tabular}

We attribute the changes in the diffusion coefficients at different temperatures to changes in the morphology of the PNIPAM chains at the QD surface. The hydrodynamic radius of the QDs depends on the conformation of the PNIPAM chains. Extended chains will increase the apparent hydrodynamic radius and as a result, the diffusion coefficient will decrease. The most pronounced changes in the diffusion coefficients upon crossing the LCST were observed for samples R10 (more than ten fold) and R1 (more than five fold). It is likely that the low grafting density of PNIPAM chains $(0.4 \%)$ for sample R25 resulted in a morphology that was less sensitive to chain collapse at $\mathrm{T}>\mathrm{LCST}$, and therefore the diffusion coefficient was less affected. 
The degrees of change in the diffusion coefficients are in good agreement as determined by both FCS and DLS techniques. For the sample R25, however, light scattering experiments showed that diffusion coefficient increased by a factor of $\sim 15$ at T $>$ LCST, while FCS results suggested that the change was not remarkable. FCS is the fluorescence-based analogue of DLS and there are a number of factors to consider before comparing the results obtained from these techniques. In DLS, the diffusion of the particles through the scattering volume and changes in the refractive index are probed, whereas in FCS fluctuations in the absorption cross-section, quantum yield and local particle concentration due to diffusion are probed. In other words, molecular brightness as well as the molecular size contributes to the observed fluctuations in FCS. In addition, DLS and FCS differ in their spatial resolution. The volume, from which the scattered light is acquired and the diffusion behavior is averaged is on the order of cubic milliliters in DLS, whereas in FCS this range is on the order of cubic microns. Therefore, the number of probed particles is much lower in FCS resulting in higher sensitivity and selectivity. Using confocal volume in FCS measurements greatly reduces the background signal, however in DLS any contaminants would contribute to the observed scattering fluctuations. As shown in Chapter 6, the absorption of the samples increases upon crossing the LCST due to PNIPAM chain collapse, which might contribute to the refractive index changes in the scattering volume and thus to the observed scattering intensities.

In summary, the FCS measurements showed that crossing the LCST resulted in changes in both optical and colloidal properties of the QD/PNIPAM hybrid structures as determined from the amplitudes and widths of the autocorrelation curves. The temperatureinduced changes in the colloidal properties were confirmed also with the DLS measurements. For further investigation of the influence of temperature on the optical properties of the samples, TCSPC measurements were performed at temperatures below and above the LCST.

The luminescence decay curves were best fitted with a tri-exponential decay function.

Figure 7.4 displays the measured decays and the corresponding fits. The goodness of the fits ( $\chi^{2}$ value) varied from 1.2 to 2.0 , which is very close to the ideal value of 1 . 


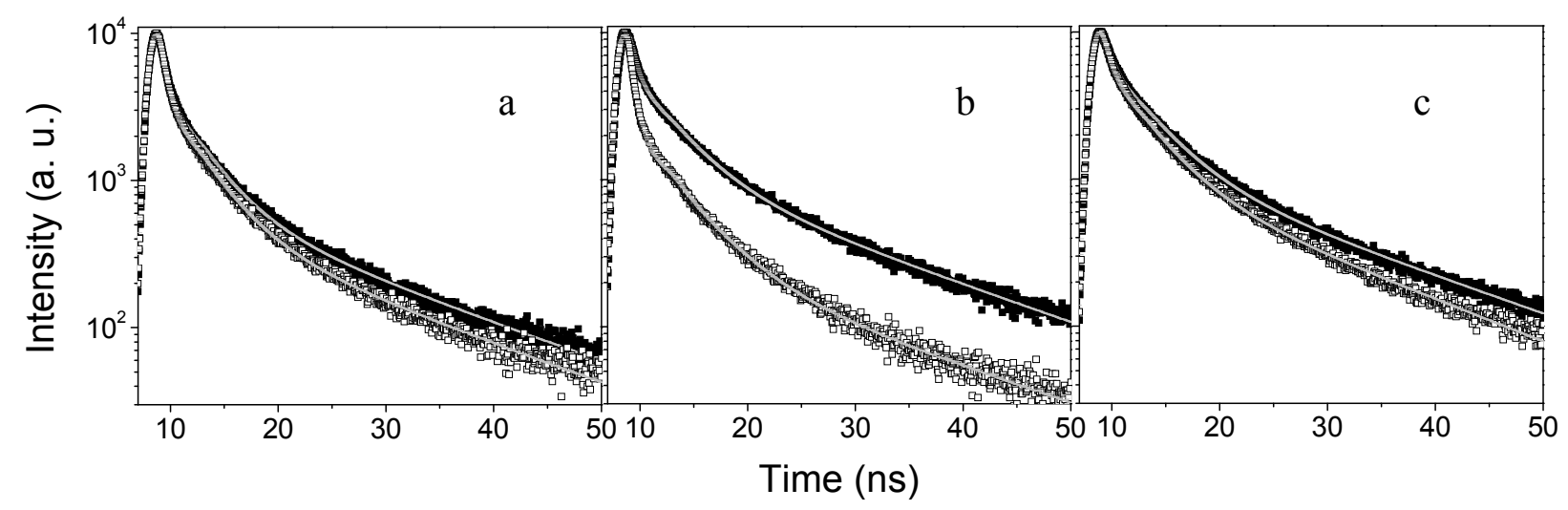

Figure 7.4. Luminescence decay curves of samples R1 (a), R10 (b) and R25 (c) measured at $\mathrm{T}<\operatorname{LCST}\left(20^{\circ} \mathrm{C}, \boldsymbol{\square}\right)$ and $\mathrm{T}>\operatorname{LCST}\left(55^{\circ} \mathrm{C}, \square\right)$ and fitted functions (gray curves).

Each sample exhibited a fast (sub-nanosecond) component $\left(\tau_{1}\right)$, which varied widely in time constant and amplitude (Table 7.2). In contrast, the second and third time constants were nearly the same for all samples: $3.5 \pm 0.5 \mathrm{~ns}$ and $16 \pm 1 \mathrm{~ns}$. When compared to the unmodified QDs, it was observed that the polymer coating process resulted in an overall decrease in the lifetimes of each sample, as well as in an increase of the relative weight of the fast component.

Sadhu et al. showed similar tri-exponential decay profiles for CdS QDs [35]. The fastest decay component was equal to $\sim 300 \mathrm{ps}$; the second and third components were $3 \mathrm{~ns}$ and $23 \mathrm{~ns}$, respectively.

Analogous to previous descriptions of QD photophysics [36], the fastest decay $(<\mathrm{ns})$ component is tentatively assigned to a trapping processes caused by surface defects or impurities. The second decay component (3-4 ns) corresponds to the presence of charged excitons and increases with the increasing laser power. The third component, which is $\sim 15$ $23 \mathrm{~ns}$, is taken to be the intrinsic lifetime, resulting from radiative electron-hole recombination. These assignments are made for unmodified QDs. In order to assess the effect of the polymer coating, we further explore the trend with temperature, length and grafting density of PNIPAM chains. 
Table 7.2. Decay components of the luminescence of R1, R 10 and R 25 and unmodified QDs (in chloroform solution) measured at $\mathrm{T}<\mathrm{LCST}\left(20^{\circ} \mathrm{C}\right)$ and $\mathrm{T}>\mathrm{LCST}\left(55^{\circ} \mathrm{C}\right)$. Each measurement has $10 \%$ uncertainty. The fastest decay components that were below the instrument response are omitted in the table $(<\mathrm{IR})$.

\begin{tabular}{lccccccc}
\hline Sample & $\tau_{1}(\mathrm{ps})$ & $\% \tau_{1}$ & $\tau_{2}(\mathrm{~ns})$ & $\% \tau_{2}$ & $\tau_{3}(\mathrm{~ns})$ & $\% \tau_{3}$ & $\tau_{\text {ave }}(\mathrm{ns})$ \\
\hline $\mathrm{R} 1\left(20^{\circ} \mathrm{C}\right)$ & 100 & 42 & 3.2 & 30 & 15.5 & 28 & $\mathbf{5 . 3}$ \\
\hline $\mathrm{R} 1\left(55^{\circ} \mathrm{C}\right)$ & $<\mathrm{IR}^{*}$ & 51 & 3.2 & 28 & 15.1 & 21 & 4.1 \\
\hline $\mathrm{R} 10\left(20^{\circ} \mathrm{C}\right)$ & 100 & 27 & 3.7 & 34 & 16.6 & 39 & $\mathbf{7 . 8}$ \\
\hline $\mathrm{R} 10\left(55^{\circ} \mathrm{C}\right)$ & $<\mathrm{IR}$ & 91 & 3.9 & 6 & 17.1 & 3 & 0.8 \\
\hline $\mathrm{R} 25\left(20^{\circ} \mathrm{C}\right)$ & 300 & 19 & 3.8 & 41 & 16.8 & 40 & $\mathbf{8 . 3}$ \\
\hline $\mathrm{R} 25\left(55^{\circ} \mathrm{C}\right)$ & 200 & 24 & 3.5 & 40 & 14.9 & 36 & 6.8 \\
\hline $\mathrm{QD} 2\left(20^{\circ} \mathrm{C}\right)$ & 460 & 13 & 4.3 & 31 & 22.2 & 56 & $\mathbf{1 3 . 8}$ \\
\hline $\mathrm{QD}\left(55^{\circ} \mathrm{C}\right)$ & 340 & 17 & 4.6 & 30 & 22.6 & 53 & 13.4 \\
\hline
\end{tabular}

To facilitate the comparison between different samples, we define an average lifetime $\left(\tau_{\text {ave }}\right)$ as the intensity $(I)$ average of the three decay components given by Eq. (7.5):

$\tau_{\text {ave }}=\sum_{i=1}^{n} I_{i} \tau_{i} / \sum_{i=1}^{n} I_{i}$

where $\tau_{\mathrm{i}}$ is the decay time of the $\mathrm{i}^{\text {th }}$ decay component with the intensity $I_{i}$. The QDs coated with the polymer having the longest PNIPAM chain (sample R25) exhibited the longest average lifetime of $8.3 \mathrm{~ns}$, whereas those coated with the shortest PNIPAM chain had $5.3 \mathrm{~ns}$ average lifetime (see Table 7.2).

Increasing the temperature above the LCST of PNIPAM resulted in a decrease in the average lifetime for all samples. The average lifetimes at T>LCST were dominated by the changes in the fastest decay component. The second and third decay components varied \pm 0.5 ns at both temperatures below and above the LCST. 
The influence of the increased temperature on the sample luminescence is twofold. First, the temperature-induced PNIPAM chain collapse can induce changes in the decay kinetics. The second aspect involves the potential influence of temperature on the intrinsic photoluminescence of QDs. In order to determine the influence of temperature on the intrinsic luminescence of QD, we performed lifetime measurements for unmodified QDs at both temperatures below and above the LCST (Table 7.2). Increasing the temperature above the LCST did not cause any substantial change in the lifetime of the QDs. In addition, we performed another control measurement using QDs modified with the same polymer coating, but the PNIPAM chains were replaced by a non-temperature-responsive polymer, poly(ethylene glycol) (PEG-QDs). The PEG-QDs showed no substantial change in their lifetimes as measured at $20{ }^{\circ} \mathrm{C}$ and $55^{\circ} \mathrm{C}$ (data not shown). These findings suggest that the observed changes in the average lifetimes of samples R1, R10 and R25 as a function of temperature are mainly due to PNIPAM chain collapse.

Lee et. al. reported the temperature dependence of fluorescence decay components of QDs, which were characterized by a bi-exponential function [37]. It was found that at temperatures above $200 \mathrm{~K}$, the slow component remained constant; however a gradual decrease in the amplitude of fast decay component accompanied with a decrease of the photoluminescence intensity was observed. This decrease was attributed to the predominance of nonradiative recombination process resulting from thermal activation. Extending this trend in the decrease of the fast decay component to our samples, we predict a decrease in the fast decay component of $11 \%$ at $55{ }^{\circ} \mathrm{C}$. The observed decrease (excluding the sample R10) is $25 \%$, i.e. considerably higher than would be expected from increased temperature alone, which also supports the idea that observed changes in the average lifetimes of the samples resulted from PNIPAM chain collapse.

To obtain more detailed insights into the mechanisms of relaxation dynamics of QD/PNIPAM hybrid assemblies requires better time resolution to capture the fastest processes. To this end, transient absorption spectroscopy, which would enable direct characterization of the charge recombination kinetics [38] could be performed.

\subsection{Conclusions}

The colloidal and optical properties of QD/PNIPAM hybrid materials at temperatures below and above the LCST of PNIPAM have been characterized. The FCS and TCSPC data provide new insights on the temperature-induced chain collapse of PNIPAM at T $>$ LCST. The colloidal and optical stability of QD/PNIPAM hybrids at T $>$ LCST of PNIPAM suggest that 
surface engineering of QDs with thermo-responsive PNIPAM chains enable realization of hybrid assemblies with switchable optical and colloidal properties. These materials are in particular attractive for in vivo applications, given that the LCST of PNIPAM is close to physiologically-relevant temperatures.

\subsection{References}

1. Henglein, A., Chem Rev, 1989. 89: p. 1861.

2. Alivisatos, A.P., Science, 1996. 271: p. 933.

3. Bruchez, M., Jr.; Moronne, M.; Gin, P.; Weiss, S.; Alivisatos, A.P, Science, 1998. 281: p. 2013.

4. Brus, L., Appl Phys A, 1991. 53: p. 465.

5. Gil, E.S.; Hudson, S.M., 2004. 29: p. 1173.

6. Schild, H.G., Prog Polym Sci, 1992. 17(2): p. 163.

7. Tomczak, N.; Janczewski, D.; Han, M.-Y.; Vancso, G.J., Prog Polym Sci, 2009. 34: p. 393.

8. Janczewski, D.; Tomczak, N.; Han, M.-Y.; Vancso, G.J., Macromolecules, 2009. 42: p. 1801.

9. Ye, J.; Hou, Y.; Zhang, G.; Wu, C., Langmuir, 2008. 24: p. 2727.

10. Zhou, L.; Gao, C.; Xu, W., J Mater Chem, 2009. 19: p. 5655.

11. Shiraishi, Y.; Adachi, K.; Tanaka, S.; Hirai, T., J Photochem Photobiol A: Chem, 2009. 205: p. 51.

12. Cheng, Z.; Liu, S.; Beines, P.W.; Ding, N.; Jakubowicz, P.; Knoll, W., Chem Mater, 2008. 20: p. 7215.

13. Doose, S.; Tsay, J.M.; Pinaud, F.; Weiss, S., Anal Chem, 2005. 77: p. 2235.

14. Murcia, M.J.; Shaw, D.L.; Long, E.C.; Naumann, C.A., Optics Commun, 2008. 281: p. 1771.

15. Zhang, P.; Li, L.; Dong, C.; Qian, H.; Ren, J., Anal Chim Acta, 2005. 546: p. 46.

16. Tagit, O.; Janczewski, D.; Tomczak, N.; Han, M.-Y.; Herek, J.L.; Vancso, G.J., Macromolecules, submitted.

17. Bacia, K.; Scherfeld, D.; Kahya, N.; Schwille, P., Biophys J, 2004. 87: p. 1034.

18. Kinjo, M., Anal Chim Acta, 1998. 365: p. 43.

19. Schwille, P., Cell Biochem Biopyhs, 2001. 34: p. 383. 
20. Sengupta, P.; Garai, K.; Balaji, J.; Periasamy, N.; Maiti, S., Biophys J, 2003. 84: p. 1977.

21. Hess, S.T.; Huang, S.; Heikal, A.A.; Webb, W.W., Biochemistry, 2002. 41: p. 697.

22. Magde, D.; Elson, E.; Webb, W.W., Phys Rev Lett, 1972. 29: p. 705.

23. Heuff, R.F.; Cramb, D.T.; Marrocco, M., Chem Phys Lett, 2008. 454: p. 257.

24. Heuff, R.F.; Swift, J.L.; Cramb, D.T., Phys Chem Chem Phys, 2007. 9: p. 1870.

25. Akcakir, O.; Therinnen, J.; Belomoin, G.; Barry, N.; Muller, J.D.; Gratton, E.; Nayfeh, M., Appl Phys Lett, 2000. 76: p. 1857.

26. Pellegrino, T.; Manna, L.; Kudera, S.; Liedl, T.; Koktysh, D.; Rogach, A.L.; Keller, S.; Rädler, J.; Natile, G.; Parak, W.J., Nano Lett, 2004. 4: p. 703.

27. Dong, C.; Huang, X.; Ren, J., Ann NY Acad Sci, 2008. 1130: p. 253.

28. Lakowicz, J.R., Principles of fluorescence spectroscopy. 2006, New York, NY: Springer.

29. Pelton, M.; Smith, G.; Scherer, N.F.; Marcus, R.A., PNAS, 2007. 104: p. 14249.

30. Xie, X.S.; Trautman, J.K., Annu Rev Phys Chem, 1998. 49: p. 441.

31. Weiss, S., Science, 1999. 283: p. 1676.

32. Yamada, T.; Goushi, K.; Xu, X.; Otomo, A., Thin Solid Films, 2008. 517: p. 1507.

33. Ito, S.; Toitani, N.; Pan, L.; Tamai, N.; Miyasaka, H., J Phys: Condens Matter, 2007. 19: p. 486208.

34. Larson, D.R.; Zipfel, W.R.; Williams, R.M.; Clark, S.W.; Bruchez, M.P.; Wise, F.W.; Webb, W.W., Science, 2003. 300: p. 1434.

35. Sadhu, S.; Patra, A., Chem Phys Chem, 2008. 9: p. 2052.

36. Salman, A.A.; Tortschanoff, A.; van der Zwan, G.; van Mourik, F.; Chergui, M., Chem Phys, 2009. 357: p. 96.

37. Lee, W.Z.; Shu, G.W.; Wang, J.S.; Shen, J.L.; Lin, C.A.; Chang, W.H.; Ruaan, R.C.; Chou, W.C.; Lu, C.H.; Lee, Y.C., Nanotechnology, 2005. 16: p. 1517.

38. Dibbell, R.S.; Watson, D.F., J Phys Chem C, 2009. 113: p. 3139. 



\section{Chapter 8}

\section{Applications of quantum dots in biosciences and medicine: Opportunities and risks}

In this chapter, applications of QDs in in-vitro labeling, in-vivo detection and photo dynamic therapy are reviewed. Potential risks related to the toxicity of $Q D$ s in biological systems are presented with the suggestions for making QDs non-toxic to humans and the environment. 



\subsection{Introduction}

It has been 40 years since the idea of manipulating matter at the molecular and atomic scales was proposed by Richard Feynman in his famous talk 'There's Plenty of Room at the Bottom' [1]. Since then, nanotechnology has evolved into a rapidly-growing applied science with a considerably high socio-economic value. The opportunities offered by nanoscale technologies and materials have high impact on a wide range of industries including medicine, electronics, and engineering [2]. By the year 2012, the nanotechnology industry is estimated to have about $\$ 1$ trillion of economic value world-wide [3]. This vast expansion and integration of nanotechnology into almost all branches of industry suggests that both human and environmental exposure to nanoscale-engineered materials will increase. However, the effects of nanomaterials on health and the environment remain largely unexplored. Lack of standard testing protocols makes it difficult to rule out potential risks of nanomaterials on human health and the environment. Therefore, it is essential that further studies explore the side effects of these nanomaterials $[4,5]$.

In this chapter, biological and medical applications of QDs are highlighted, with a special emphasis on related toxicology studies.

\subsection{Applications of quantum dots in biology and medicine}

The unique optical and electronic properties, combined with the possibility of tuning these properties, make QDs especially valuable for biomedical imaging [6, 7]. Recent progress achieved in the design and fabrication of various platforms of QD-based materials has enabled realization of many applications of such materials.

Surface engineering strategies have been widely used to render QDs suitable for biological applications. The basic requirements involve water solubility and the ability of bioconjugation. There are two general strategies for rendering QDs water soluble. The first method involves exchanging the surface-capping organic hydrophobic ligand with a water soluble ligand (Figure 8.1.a). The hydrophilic stabilizers should be able to strongly attach to the QD surface to maintain colloidal stability. Bifunctional ligands with a thiolate group at one end and a hydrophilic group at the other end, such as mercaptoacetic acid, are most commonly used in the ligand exchange reactions [8]. The second strategy for making QDs water soluble is to coat them with an amphiphilic polymer without removing the hydrophobic capping ligand (Figure 8.1.b). Several polymers have been used, such as PEG-derivatized phospholipids [9], octylamine-modified polyacrylic acid [10], and amphiphilic block copolymers [11]. 
a Bifunctional linkage

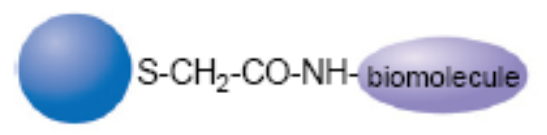

b Hydrophobic attraction

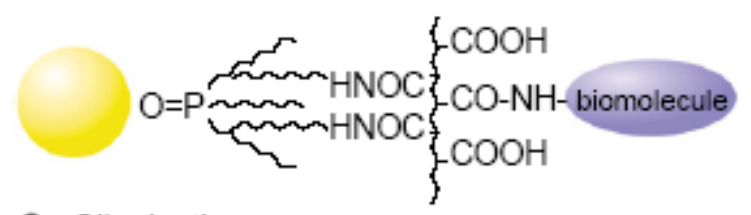

C Silanization

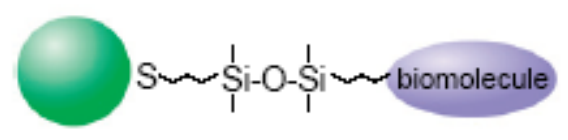

d Electrostatic attraction

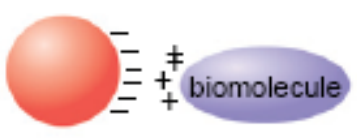

e Nanobeads

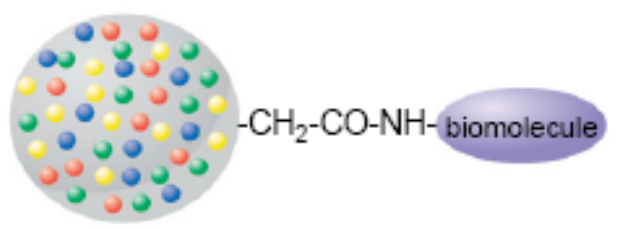

Figure 8.1. Schematic presentation of different approaches used for conjugation of QDs to biomolecules: a) Attachment of a biomolecule to QD surface by using a bifunctional ligand such as mercaptoacetic acid. b) Binding of a biomolecule to QD surface modified with acrylic acid polymer via hydrophobic interactions. c) Solubilization and bioconjugation of QDs using mercapto-silane compound. d) Linking negatively charged QDs to positively charged biomolecules via electrostatic interactions. e) Incorporation of QDs within nanobeads with functional groups on the surface and conjugation to biomolecules. This image was taken from [12].

A number of ligands that were used to make QDs water soluble have also been reported to enable conjugation of QDs to biomolecules. Figure 8.1 shows the conjugation strategies commonly used for attaching proteins to the QD surface. The different conjugation strategies suggest that the water solubilization and conjugation processes will result in modified colloidal properties of the QDs including surface charge and hydrodynamic size. Therefore, each strategy needs to be tailored depending on the nature of the target biological system [13]. For example, the resulting increase in the size of QDs may make the intracellular delivery difficult and could increase cytotoxicity [14]. In addition, it is also a challenge to control the number and orientation of biomolecules attached to QD surface [12]. These and other important technical issues regarding the surface coating chemistry still remain to be solved, especially when in vivo conditions are considered. The surface coating 
chemistry should be designed in a way that it provides minimal non-specific binding while maintaining stability, avoiding oxidation and resisting salt concentration levels within the cells.

\subsubsection{Applications of quantum dots as in vitro fluorescent labels}

One of the most common visualization techniques used in cell biology is fluorescent labeling, largely because cells are mostly transparent to visible light and their compartments are too small to be observed directly $[15,16]$. The idea is to chemically attach a fluorescent probe to the receptor molecules that specifically and selectively bind to desired cell component. First studies on using bioconjugated QDs for bio-imaging were reported by Bruchez et al. [17] and Chan and Nie [18]. These studies represented a breakthrough in applications of QDs in biological systems. Since then, there has been a growing interest in improving the cellular targeting of modified QDs. A variety of examples are highlighted below: DNA-mediated arrangement of QDs on a surface via complementary nucleotide binding is presented in Figure 8.2. QDs of two different sizes are conjugated with two different oligonucleotide sequences. The complementary sequences of these oligonucleotides are immobilized on different substrates. Upon incubation of the substrates with a mixed solution of QDs, only those modified with the corresponding complementary sequences attach to the surface [19].

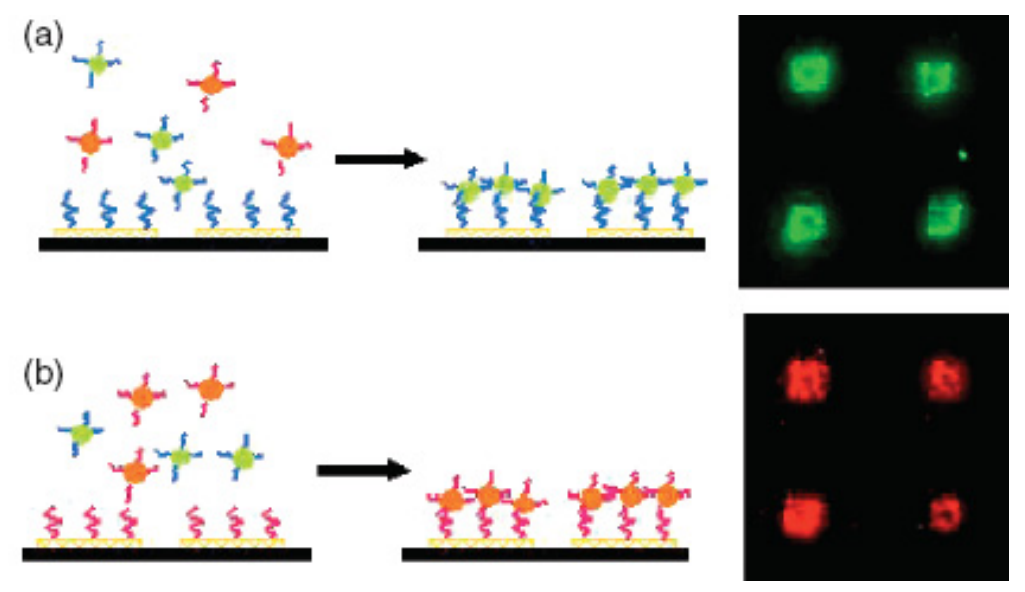

Figure 8.2. Green and red emitting QDs are modified with different oligonucleotide sequences. The substrate is modified with complementary sequences to green (a) and to red (b) QD-oligonucleotide conjugates. After incubation and several washing steps, only the QDs conjugated to oligonucleotides that have the complementary sequence to those immobilized on the surface bind. This image was taken from [19]. 
Several other research groups attached QDs directly to a biomolecule of interest. Zhang et al. reported a conjugation scheme of a ribosome-inactivating protein (trichosanthin, TCS) to mercapto-modified QDs [20] for studying the uptake, localization and distribution of TCS-QDs in cancerous cells.

The strongest known biological non-covalent interaction is between avidin and biotin molecules $\left(\mathrm{K}_{a}=10^{15}\right)$ [21]. Avidin is a tetrameric glycoprotein that is soluble in aqueous solutions and is stable over a wide range of $\mathrm{pH}$ and temperature. Biotin is a vitamin that plays a role in cell growth, the production of fatty acids and in fat and amino acid metabolism. The high affinity binding of avidin to biotin provides an excellent method for labeling and targeting. Streptavidin, isolated from Streptomyces avidinii, was used to modify QDs for labeling biotinylated F-actin fibres [10] (Figure 8.3).

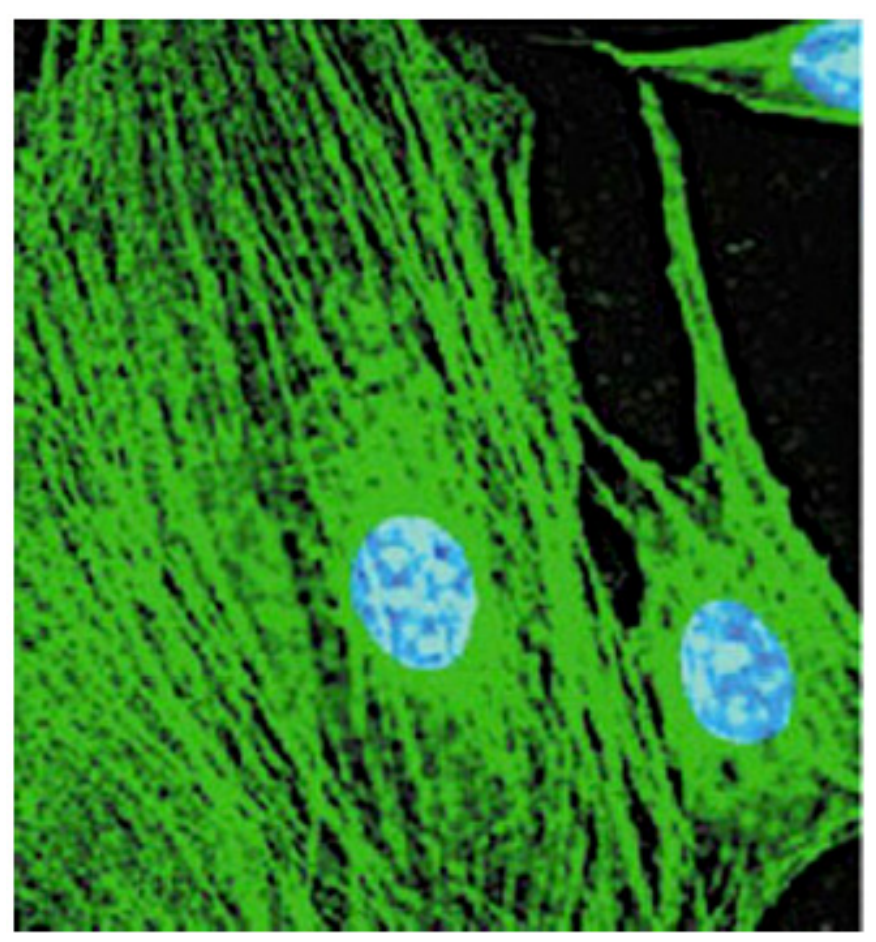

Figure 8.3. Fluorescence image of mouse $3 \mathrm{~T} 3$ fibroblasts. Actin filaments are stained with biotinylated phalloidin and streptavidin-modified QDs. The nuclei are counterstained with Hoechst blue dye. This image was taken from [10].

In another study, streptavidin-conjugated QDs were used for chromosomal detection using fluorescence in situ hybridization [22] with biotinylated single stranded DNA (ssDNA) as probes. Although the brightness and the resistance to photobleaching was a clear advantage of QDs for quantitative analysis, blinking and intermittency of QD fluorescence 
was also observed. The blinking behavior was attributed to surface chemistry and the environment of QDs [23].

As mentioned in Chapter 2, one major advantage of QDs is their broad absorption and narrow-band emission profiles, which make multiplex detection possible. Mattheakis et al. recently reported a QD system for encoding, imaging, and decoding single cells for multiplexing and other assay applications [24] (Figure 8.4). The multicolor QDs were delivered to different mammalian cells and each cell type was encoded separately with a unique and spectrally resolvable QD code.

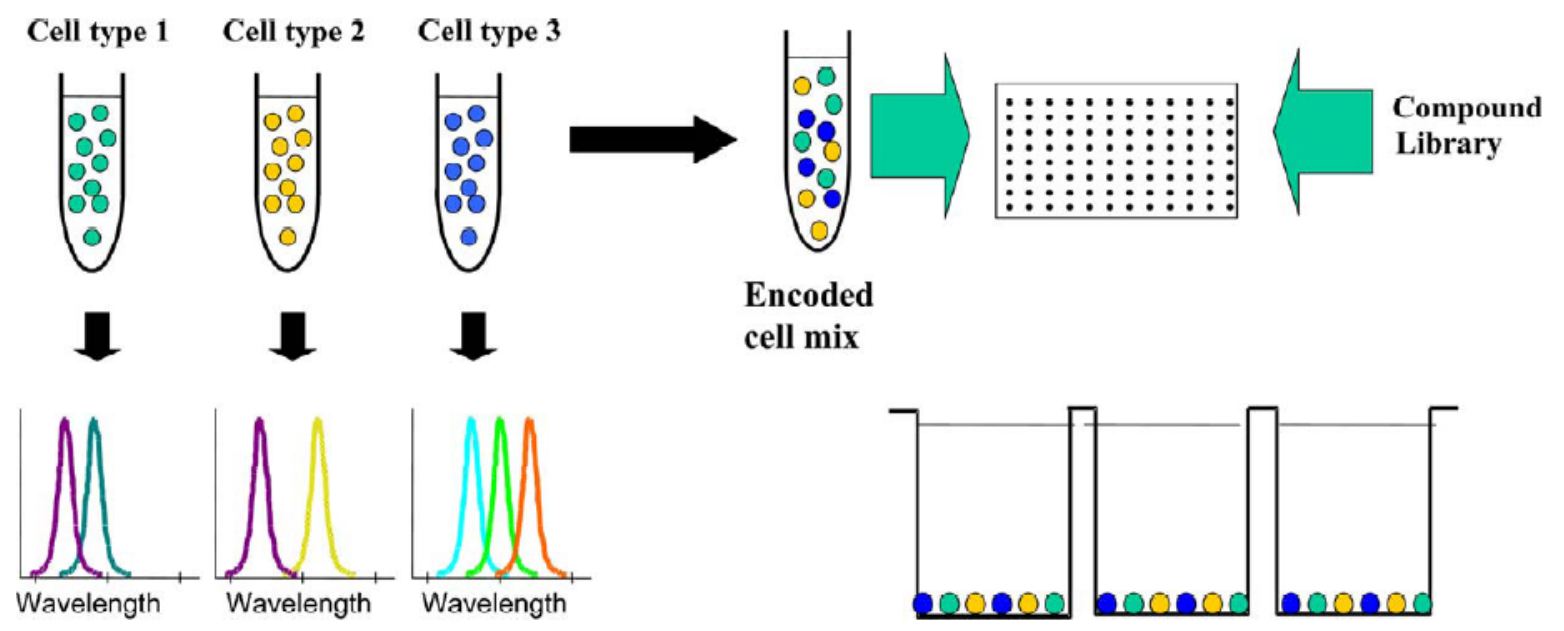

Figure 8.4. Schematic illustration of a multiplex cellular screening assay using QDs. Different cell lines are encoded separately with specific QD cell codes. The cells are mixed and aliquots from the master mix are deposited into the wells of the assay plate. The compounds are also added to the assay plate and the plate is imaged using fluorescence microscope. This image was taken from [24].

Five separate cultures of mammalian cells were encoded with 566, 582, 608, 630 and $647 \mathrm{~nm}$ QDs. The fluorescence microscopy image of the mixed cell population and the corresponding resolved spectra are shown in Figure 8.5. 

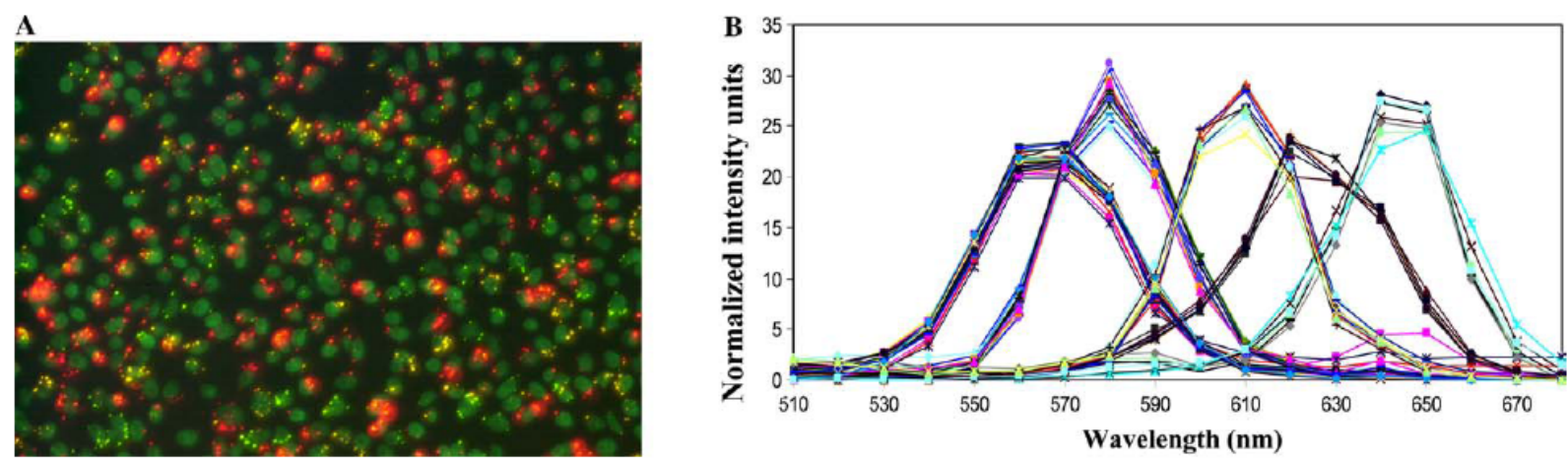

Figure 8.5. A. Fluorescence microscopy image of a mixed cell population encoded with different QDs. B. The normalized emission spectra of around 60 cells from the mixed cell population. Each emission wavelength for each QD-type is resolved. This image was taken from [24].

With the use of five different colors of QDs and the three different intensity levels for each, hundreds of unique codes can be created.

\subsubsection{Applications of quantum dots for in vivo imaging}

Using QDs for in vivo imaging is still in the early stages of development. One of the first studies of QDs as in vivo labeling agents was reported by Dubertret et al [9]. QDs encapsulated in phospholipid block copolymer micelles were injected to Xenopus embryos and were visualized using bright field and fluorescence imaging (Figure 8.6). When injected into Xenopus embryos, the QD-micelles were reported to be stable, non-toxic, and resistant to photobleaching.

Other studies of in vivo labeling using QDs involved targeting tumors [25-27], or imaging vasculature in different tissues [28]. Morgan et al. reported real time in vivo optical imaging of a tumor in mice [29]. Near infrared (NIR) emitting QDs were coated with bovine serum albumin (BSA) and were injected either subcutaneously or intravenously into mice (Figure 8.7). The QDs used in this study were proven to be useful as contrast agents as there was no significant photobleaching or degradation of the QDs after continuous excitation for 1 hour. 


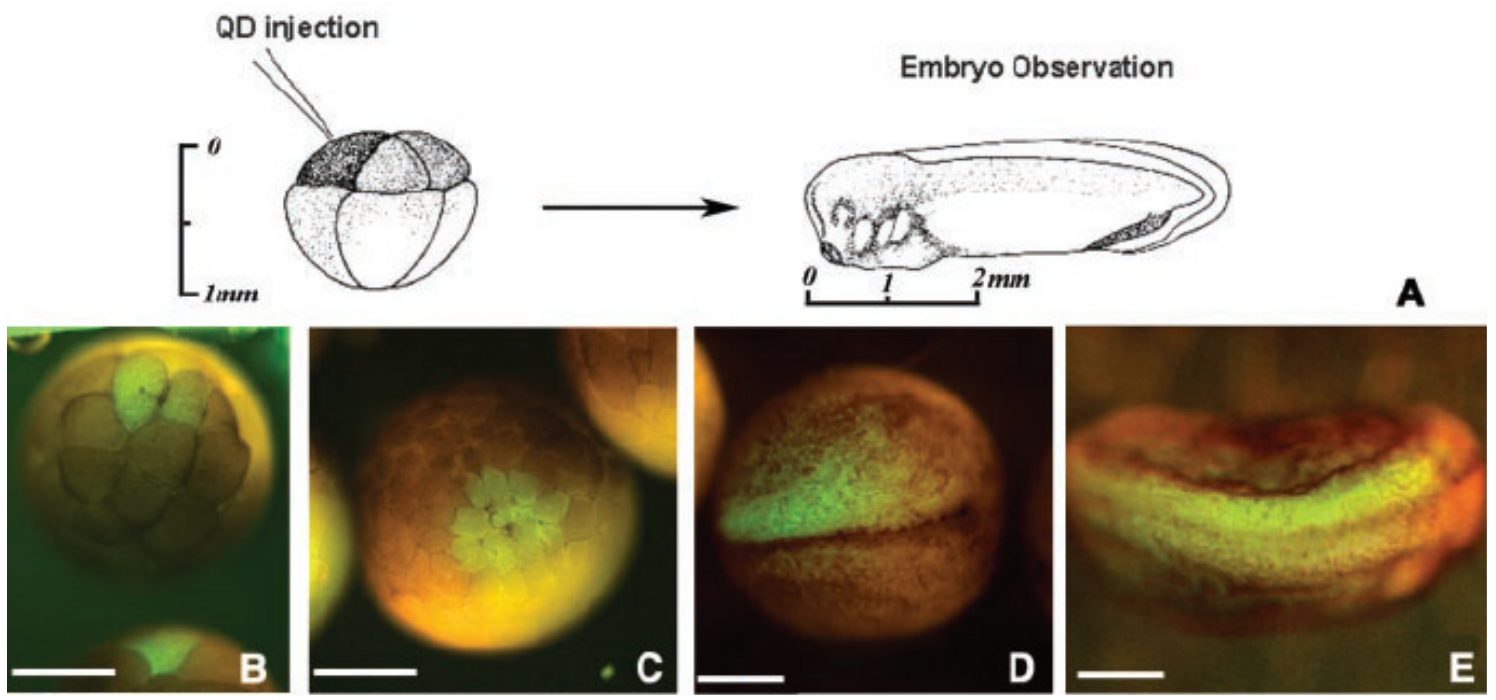

Figure 8.6. Labeling of Xenopus embryos using QDs at different stages of development. A) Injection of QDs embedded within phospholipids block copolymer micelles to an individual blastomere during early stages of development. B) Injection of one cell of eight-cell-stage embryo results in individual labeling of blastomeres. C) The same embryo as in B, after 1 hour. The daughter cells are labeled with QDs. D) and E) show the later stages of development. The QDs are visible through the pigmented layer of epidermis. Scale bars: 0.5 $\mathrm{mm}$. This image was taken from [9].
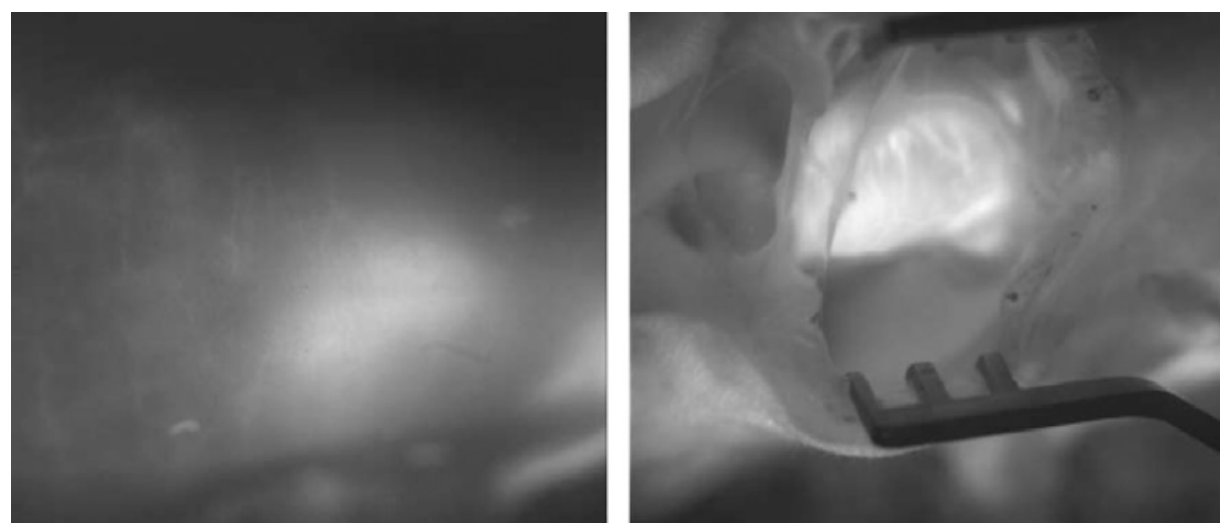

Figure 8.7. Thoracic and abdominal regions of a mouse imaged after injection of QDs before (left) and after (right) the incision. The image was taken from [29].

In another study, QDs were modified with a number of marker proteins for specific targeting to lung, blood vessels or lymphatic vessels in tumors [25] in mice. The schematic presentation of QD targeting is shown in Figure 8.8. 

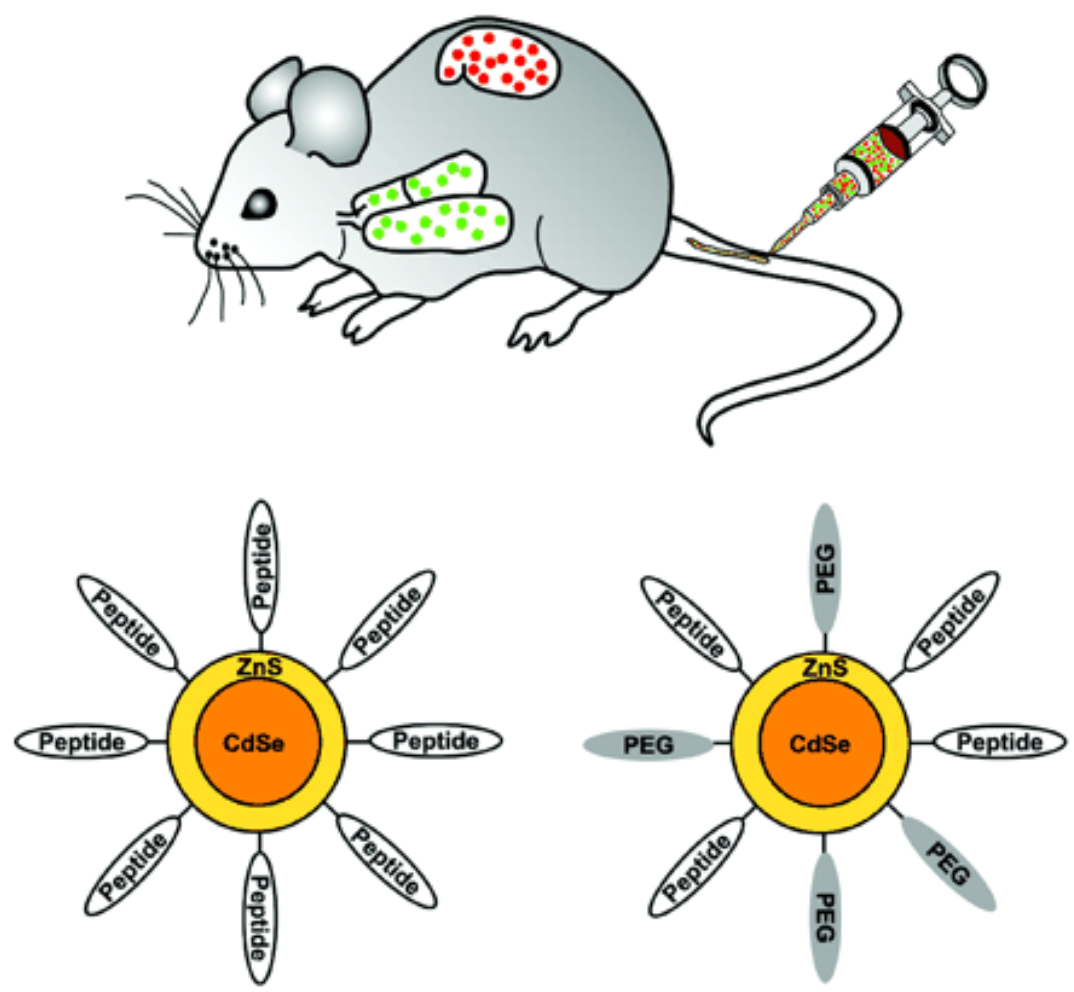

Figure 8.8. The upper scheme shows the intravenous injection of QDs. The lower scheme shows the design of the surface coating of QDs used for targeting. The QDs were coated with either peptides only, or, peptides and PEG. PEG helps maintaining the colloidal stability in aqueous environment and minimizes nonspecific binding. This image was taken from [25].

The injected QDs were observed to accumulate in the targeted tissues. The Figure 8.9 demonstrates the resulting fluorescence images.
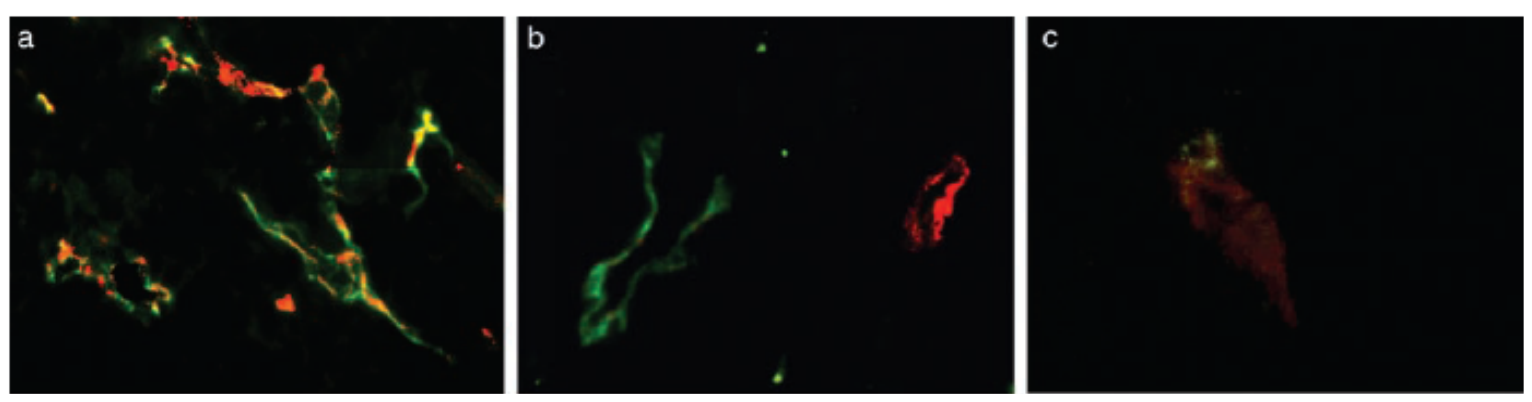

Figure 8.9. Selective binding of QDs to targeted tissues. The blood vessels are visualized coinjecting tomato lectin (green). a) Colocalization of QDs with lectin in the blood vessels in the tumor tissue. b) QDs with a different surface coating accumulated in the blood vessel but not colocalized with the blood vessel marker. c) Red and green QDs with different targets injected into the same tumor. This image was taken from [25]. 
Attaching PEG to the QD surface in addition to targeting proteins prevents the nonselective accumulation of QDs in the tissues. When compared to organic dyes, tissue penetration of QDs was reduced, which was likely a result of the relatively large size of the PEG-modified QDs. Nonetheless, these results encourage the construction of more complex nanostructures with simultaneous capabilities for recognition, sensing and drug delivery.

At present, there is relatively little work published on QDs as fluorescent markers for whole body imaging. One of the most important difficulties is the potential toxicity of the QDs, which will be discussed in detail in the following sections. Other challenges for in vivo imaging using QDs include absorbance and scattering by the tissues and autofluorescence [12]. The absorption and scattering by the tissue is much lower in the near-infrared region (NIR, $700-1000 \mathrm{~nm}$ ) [28], therefore QDs emitting at NIR would be better labels for in vivo applications.

\subsubsection{Applications of quantum dots in photodynamic therapy of cancer}

Photodynamic therapy (PDT) is regarded as a new modality in the treatment of cancer, though it was first introduced in the 1980s [30, 31]. The basic idea in PDT is to combine a photosensitizing agent and light. Both light and the photosensitizer are harmless by themselves; however, their combination in the presence of oxygen can result in selective tumor destruction. The concept of PDT is shown in Figure 8.10.
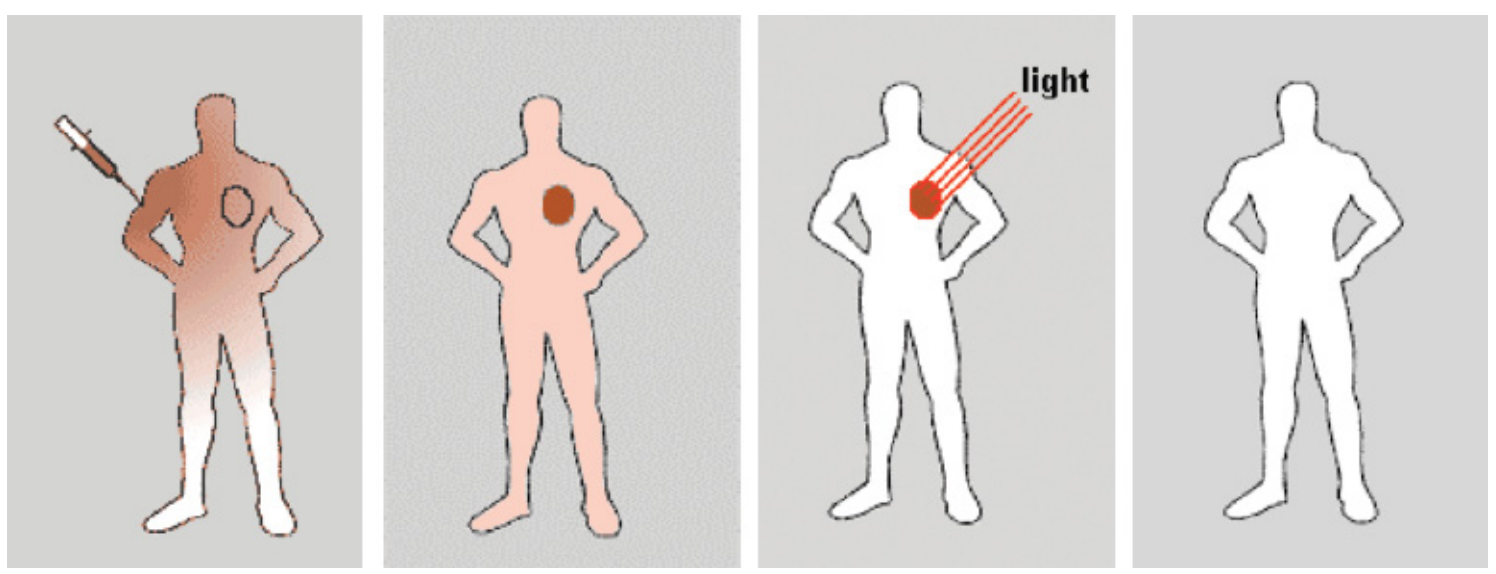

Figure 8.10. a) The photosensitizing drug is administrated intravenously to the patient. b)

The sensitizer is localized in the tissue bearing the tumor. c) The tumor area is irradiated with light of appropriate wavelength for a predetermined time. d) After several sessions, the tumor disappears. This image was taken from [21]. 
In PDT the singlet oxygen that is produced by the sensitizer destroys the neighboring tissue. With proper localization of the sensitizer within the tumor tissue, the singlet oxygen can be confined to the targeted area. The efficiency of singlet oxygen generation determines the effectiveness of the PDT. The most commonly used photo sensitizers include porphyrins, chlorins, and bacteriochlorins [21]. Recently, Samia et al. reported two strategies for the use of QDs as PDT agents [32]. The first approach involved using QDs directly as photosensitizers (Figure 8.11). In the second approach QDs were used to excite a conventional photosensitizer, phthalocyanine ( $\mathrm{Pc} 4)$.

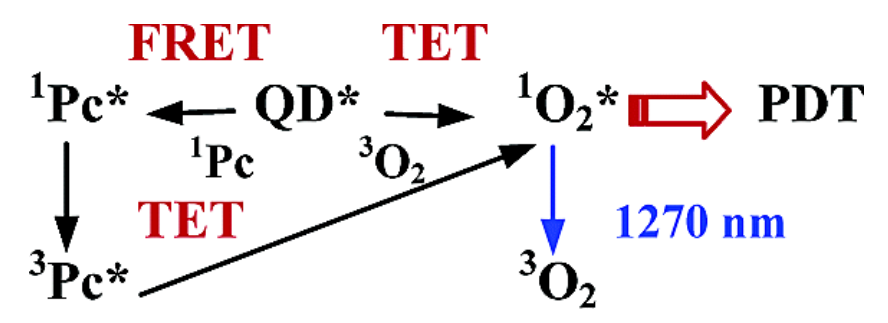

Figure 8.11. Schematic illustration of singlet oxygen generation through QD-excitation. In the first case (right) the QDs are used directly as photosensitizers. Singlet oxygen is generated via a triplet energy transfer (TET) process. In the second case (left), the QD is used as an energy transfer agent to excite a photosensitive Pc4 molecule via fluorescence resonance energy transfer (FRET), which then generates the singlet oxygen via the triplet energy transfer process. This image was taken from [32].

The basic requirements for QDs to be used in PDT for photo-sensitizer enhancement are, (i) the QD should have an emission spectrum that matches the absorption spectrum of the sensitizer; (ii) the luminescence efficiency of QDs should be high; (iii) the QD should be easily linked to the sensitizer; (iv) and the QD should be non-toxic, water soluble and stable in biological environment [21].

The proposed mechanisms of PDT induced by QDs are shown in Figure 8.12. 


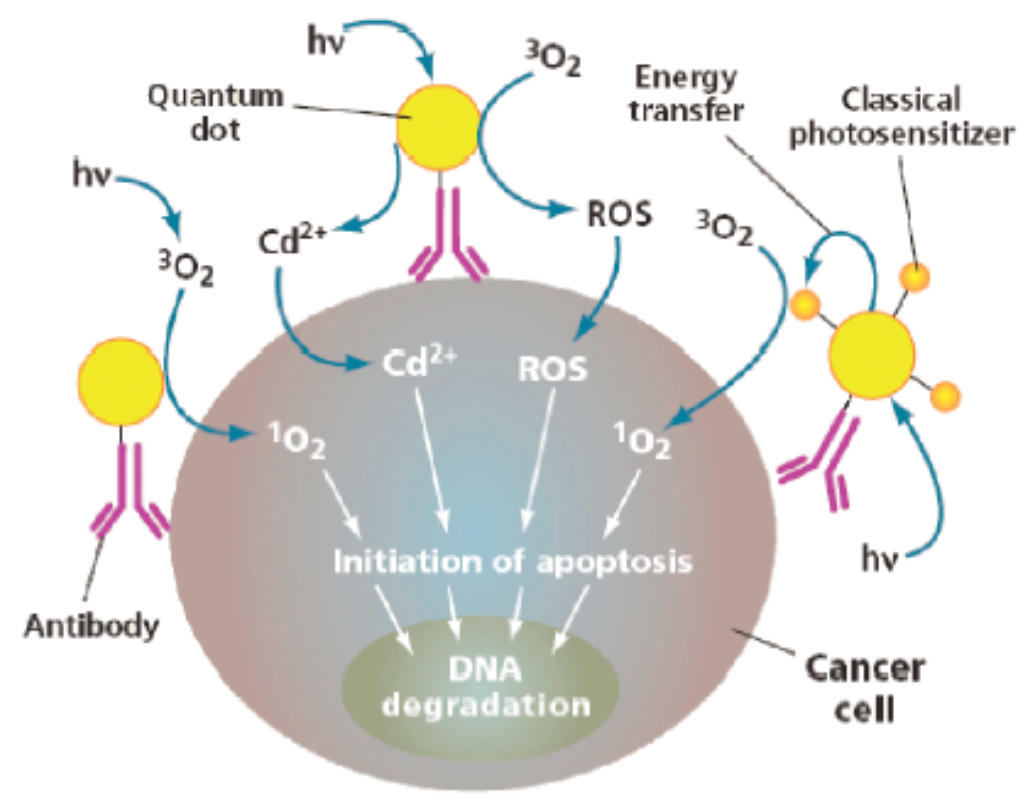

Figure 8.12. The PDT mechanisms induced by the QDs. QDs or QD-photo-sensitizer complexes labeled with antibodies are targeted to tumor tissue. Excited QDs may directly induce the formation of reactive oxygen species (ROS), or, they might transfer their energy to the photo-sensitizers. Free cadmium ions or singlet oxygen species induce apoptosis (programmed cell death). This image was taken from [21].

Figure 8.12 shows the possible mechanisms of PDT that the QD/sensitizer conjugates can induce. Using QDs in combination with photo sensitizers provides flexibility in using variable excitation wavelengths to activate the sensitizer molecule. QDs are also known to generate other active oxygen and nitrogen species. For instance presence of CdSe/ZnS QDs resulted in increased nitric oxide generation during the photolysis of water [33]. In addition, it was also proposed that QDs exposed to UV radiation release $\mathrm{Cd}^{2+}$ and $\mathrm{Se}^{2+}$ ions that might generate oxidative stress in living cells [34].

So far, the above mentioned findings show that QDs have been successfully applied to biological systems for labeling, imaging and therapeutic purposes. Then the question arises: how safe is it to use QDs in biological systems?

\subsection{QD toxicity}

Exposure of cells to cytotoxic compounds may result in: i) decrease in cell viability, where the cells stop actively growing and dividing; ii) cell necrosis, where the cells loose the membrane integrity and die as a result of cell lysis; iii) cell apoptosis, where the cells activate 
a genetic code for programmed cell death [35]. A compound which causes at least one of the three cases is referred to as being cytotoxic.

Some in vitro studies have suggested that certain types of QDs may be cytotoxic [8, $11,34,36,37]$. The extent of cytotoxicity has been found to depend on a number of factors such as size, capping material, dose of QDs, surface chemistry, and processing parameters $[36,38]$.

A number of mechanisms have been suggested to be responsible for the QD cytotoxicity. These mechanisms include desorption of free cadmium (core degradation) to the surrounding tissue, free radical formation, and interaction of QDs with cellular organelles [34]. Derfus et al. studied QD cytotoxicity using a hepatocyte culture. Exposure of CdSe QDs to an oxidative environment caused decomposition of the QD core and detachment of cadmium ions. High levels of $\mathrm{Cd}^{2+}$ were found both in the air-oxidized sample (126 ppm) and UV-exposed sample (82 ppm), whereas the non-oxidized CdSe solution contained only 6 ppm of $\mathrm{Cd}^{2+}$. The cytotoxicity rates observed for oxidized and non-oxidized CdSe QDs were $95-98 \%$ and $0 \%$, respectively. The same group studied also the influence of the surface coating of the QDs on their cytotoxicity. They coated the CdSe cores with ZnS shells and modified the surface by attaching bovine serum albumin (BSA). The CdSe/ZnS QDs had $66 \%$ cell viability, whereas this number increased to $98 \%$ for $\mathrm{CdSe} / \mathrm{ZnS}$ QDs coated with BSA, which shows that successive addition of an inorganic layer and organic ligands minimizes the cytotoxicity of QDs.

Lovric et al. reported that CdTe QDs with mercaptopropionic acid (MPA) and cysteamine coatings were toxic to rat cell cultures in the $10 \mu \mathrm{g} / \mathrm{mL}$ concentration range, however this range dropped down to $1 \mu \mathrm{g} / \mathrm{mL}$ when uncoated QDs were used [36]. The size of the QDs was also found to influence the cytotoxicity. At equal concentrations, cytotoxicity was found to be more pronounced for smaller QDs $(2.2 \pm 0.1 \mathrm{~nm})$ than bigger QDs $(5.2 \pm 0.1$ $\mathrm{nm})$. The size of QDs was also observed to affect the distribution of QDs within the cell, such that smaller QDs were localized in the nuclear compartment whereas the larger QDs were localized in the cytosol.

Hoshino et al [39] reported a cytotoxicity study where the capping materials of QDs, mercaptoundecanoic acid (MUA) and cysteamine, were tested in the absence of core QDs. MUA was reported to cause severe cytotoxicity in the rat cells at $100 \mu \mathrm{g} / \mathrm{mL}$ concentration after 12 hours of exposure. Cysteamine of the same concentration was tested for the same duration and was found to be weakly toxic. Hoshino et al. attributed the cytotoxicity to the capping material rather than to the QD itself. In a similar study reported by Shiohara et al, it 
was observed that MUA-coated $\mathrm{CdSe} / \mathrm{ZnS}$ QDs were cytotoxic to HeLa cells and primary human hepatocytes at $100 \mu \mathrm{g} / \mathrm{mL}$ concentration [38].

There have been a number of in vitro and in vivo studies of QDs reporting lack of evidence for QD-induced cytotoxicity [9, 40-43]. However, some of the reports suggest that QDs may affect cell growth and viability at certain concentrations. For instance, Dubertret et al. reported that QDs with PEG surface coatings resulted in cell abnormalities when injected into Xenopus blastomeres at concentrations of $5 \times 10^{9}$ QDs/cell, whereas those that were injected with $2 \times 10^{9}$ concentrations displayed a normal phenotype [9]. The dose dependency was also shown in a study by Hoshino et al. where the cell viability decreased at QD concentrations above $0.1 \mathrm{mg} / \mathrm{mL}$ [39]. After 10 days of incubation, the fluorescence intensity was observed to concentrate in the endosomes, suggesting intracellular QD degradation. Interestingly, in vivo studies with the same QDs showed no evidence for toxicity when injected to mice intravenously.

Ballou et al. intravenously injected QDs that were coated with an amphiphilic polymer and conjugated with PEG into mice [40]. The concentration of QDs was chosen to be 20 picomol of QD per gram of animal weight. There were no signs of necrosis at the sites of tissue deposition and the mice were viable until the necropsy. In addition, no signs of in vivo QD degradation were observed in TEM studies performed after necropsy. The in vivo QD stability was attributed to the presence of the amphiphilic coating.

Overall, the reports mentioned here suggest that QD toxicity depends on the combination of many factors including QD size, charge, and concentration, coating material and oxidative, photolytic and colloidal stability. The nature of the functional surface coating and QD core stability are also significant factors influencing cytotoxicity.

\subsection{Conclusions}

With the recent advances in nano-fabrication technologies it is very likely that the use of QD products will increase. It is necessary to elucidate the potential adverse effects of these materials to the human health and the environment. Given the potential benefits offered by the QD technology, clarifying the mechanisms and sources of QD toxicity will help avoid problems that could be encountered due to misapplication of this technology. Only with this knowledge it will be possible to achieve a QD technology that is compatible with the social and ecologic systems. 


\subsection{References}

1. Feynman, R.P., Plenty of Room at the Bottom 1956, Presentation to American Physical Society.

2. Hardman, R., Environ Health Pers, 2006. 114: p. 165.

3. Service, R.F., Science, 2004. 304: p. 1732.

4. Colvin, V.L., Nat Biotechnol, 2003. 21: p. 1166.

5. Oberdorster, G.; Oberdorster, E.; Oberdorster, J., Environ Health Perspect, 2005. 113: p. 823.

6. Alivisatos, P., Nat Biotechnol, 2004. 22: p. 47.

7. Chan, W.C.W.; Maxwell, D.J.; Gao, X.; Bailey, R.E.; Han, M.; Nie, S., Curr Opin Biotechnol 2002. 13: p. 40.

8. Medintz, I.L.; Uyeda, H.T.; Goldman, E.R.; Mattoussi, H., Nat Mater, 2005. 4: p. 435.

9. Dubertret, B.; Skourides, P.; Norris, D.J.; Noireaux, V.; Brivanlou, A.H.; Libchaber, A., Science, 2002. 298: p. 1759.

10. Wu, X.; Liu, H.; Liu, J.; Haley, K.N.; Treadway, J.A.; Larson, J.P.; Ge, N.; Peale, F.; Bruchez, M.P., Nat Biotechnol, 2002. 21: p. 41.

11. Kirchner, C.; Liedl, T.; Kudera, S.; Pellegrino, T.; Javier, A.M.; Gaub, H.E.; Stölzle, S.; Fertig, N.; Parak, W.J., Nano Lett, 2004. 5: p. 331.

12. Jamieson, T.; Bakhshi, R.; Petrova, D.; Pocock, D.; Imani, M.; Seifalian, A.M., Biomaterials, 2007. 28: p. 4717.

13. Luccardini, C.; Tribet, C.; Vial, F.; Marchi-Artzner, V.; Dahan, M., Langmuir, 2006. 22: p. 2304.

14. Weng, J.F.; Ren, J.C., Curr Med Chem, 2006. 13: p. 897.

15. Enenkel, C.; Lehmann, A.; Kloetzel, P.M., Mol Biol Rep, 1999. 26: p. 131.

16. Gao, X.; Chan, W.C.; Nie, S., J Biomed Opt, 2002. 7: p. 532.

17. Bruchez, M.; Moronne, M.; Gin, P.; W eiss, S.; Alivisatos, A.P., Science, 1998. 281: p. 2013.

18. Chan, W.C.W.; Nie, S.M., Science, 1998. 281: p. 2016.

19. Parak, W.J.; Gerion, D.; Pellegrino, T.; Zanchet, D.; Micheel, C.; Williams, S.C.; Boudreau, R.; Le Gros, M.A.; Larabell, C.A.; Alivisatos, A.P. , Nanotechnology, 2003. 14: p. R15.

20. Zhang, C.Y.; Ma, H.; Nie, S.M.; Ding, Y.; Jin, L.; Chen, D.Y., Analyst, 2000. 125: p. 1029.

21. Chen, W., J Nanosci Nanotechnol, 2008. 8: p. 1019. 
22. Xiao, Y.; Barker, P.E., Nucleic Acids Res, 2004. 32: p. e28.

23. Shimizu, K.T.; Neuhauser, R.G.; Leatherdale, C.A.; Empedocles, S.A.; Woo, W.K.; Bawendi, M.G., Pyhs Rev B, 2001. 63: p. 205316.

24. Mattheakis, L.C.; Dias, J.M.; Choi, Y.J.; Gong, J.; Bruchez, M.P.; Liu, J.Q.; Wang, E., Anal Biochem, 2004. 327: p. 200.

25. Akerman, M.E.; Chan, W.C.W.; Laakkonen, P.; Bhatia, S.N.; Ruoslahti, E., PNAS, 2002. 99: p. 12617.

26. Gao, X.H.; Cui, Y.Y.; Levenson, R.M.; Chung, L.W.K.; Nie, S.M., Nat Biotechnol, 2004. 22: p. 969.

27. Chen, L.M.; Zurita, A.J.; Ardelt, P.U.; Giordano, R.J.; Arap, W.; Pasqualini, R., Chem Biol, 2004. 11: p. 1081.

28. Lim, Y.T.; Kim, S.; Nakayama, A.; Stott, N.E.; Bawendi, M.G.; Frangioni, J.V., Mol Imaging, 2003. 2: p. 50.

29. Morgan, N.Y.; English, S.; Chen, W.; Chernomordik, V.; Russo, A.; Smith, P.D.; Gandjbakhche, A. , Acad Radiol, 2005. 12: p. 313.

30. Dougherty, T.J.; Gomer, C.J.; Henderson, B.W.; Jori, G.; Kessel, D.; Korbelik, M.; Moan, J.; Peng, Q., J Nation Cancer Inst, 1998. 90: p. 889.

31. De Rosa, F.S.; Bentley, M.V., Pharm Res, 2000. 17: p. 1447.

32. Samia, A.C.S.; Chen, X.B.; Burda, C., J Am Chem Soc, 2003. 125: p. 15736.

33. Neuman, D.; Ostrowski, A.D.; Mikhailovsky, A.A.; Absalonson, R.O.; Strouse, G.F.; Ford, P.C., J Am Chem Soc, 2008. 130: p. 168.

34. Derfus, A.M.; Chan, W.C.W.; Bhatia, S.N., Nano Lett, 2004. 4: p. 11.

35. Tsay, J.M.; Michalet, X., Chem Biol, 2005. 12: p. 1159.

36. Lovric, J.; Bazzi, H.S.; Cuie, Y.; Fortin, G.R.A.; Winnik, F.M.; Maysinger, D., J Mol Med, 2005. 83: p. 377.

37. Hsieh, S.C.; Wang, F.F.; Hung, S.C.; Chen, Y.; Wang, Y.J., J Biomed Mater Res Part B-Appl Biomater, 2006. 79B: p. 95.

38. Shiohara, A.; Hoshino, A.; Hanaki, K.; Suzuki, K.; Yamamoto, K., Microbiol Immunol, 2004. 48: p. 669.

39. Hoshino, A.; Fujioka, K.; Oku, T.; Suga, M.; Sasaki, Y.F.; Ohta, T.; Yasuhara, M.; Suzuki, K.; Yamamoto, K., Nano Lett, 2004. 4: p. 2163.

40. Ballou, B.; Lagerholm, B.C.; Ernst, L.A.; Bruchez, M.P.; Waggoner, A.S., Bioconjug Chem, 2004. 15: p. 79. 
41. Hoshino, A.; Hanaki, K.; Suzuki, K.; Yamamoto, K., Biochem Biophys Res Commun, 2004. 314: p. 46.

42. Jaiswal, J.K.; Mattoussi, H.; Mauro, J.M.; Simon, S.M., Nat Biotechnol, 2003. 21: p. 47.

43. Voura, E.B.; Jaiswal, J.K.; Mattoussi, H.; Simon, S.M., Nat Med, 2004. 10: p. 993. 


\section{Summary}

Quantum dots, QDs, receive growing attention from many research disciplines owing to their advantages as fluorescent probes including their nanoscale size (similar to biomolecules), high quantum yield and molar extinction coefficients, versatility in surface modification, broad excitation spectra (for multicolor imaging) and narrow band emission and tunable optical properties. Fabricating QD/polymer hybrid nanostructures enables realization of many potential applications as optoelectronic devices, biological sensors, and photonic structures because encaging QDs within polymer matrices not only enables the control over optical and spectroscopic properties of QDs but also introduces a strong resistance to chemical and photodegradation. The research described in this thesis aims at synthesis and characterization of $\mathrm{CdSe} / \mathrm{ZnS}$ core/shell QDs, synthesis and characterization of temperature-responsive polymer matrices made of poly( $N$-isopropylacryl amide), PNIPAM, as carriers of QDs, and fabrication of QD/PNIPAM assemblies with potential applications as sensing devices to be used in bio-nanotechnology.

Chapter 1 was a general introduction to this thesis. Chapter 2 provided a basic background in the optical properties of QDs and reviewed the recent developments on combining stimuli responsive polymers with QDs via different approaches, including layerby-layer deposition, macromolecular grafting and in situ QD synthesis within polymeric matrices.

Chapter 3 described the basics of some of the microscopy and spectroscopy techniques used for the characterization of QDs down to the single molecule level. In addition to atomic force microscopy and confocal optical microscopy, combined microscopy techniques for studying QDs and QD-bearing systems were explained. Spectroscopy techniques that were mentioned in this chapter included fluorescence correlation spectroscopy and time correlated single photon counting. The microscopy and spectroscopy techniques mentioned are useful tools for characterization of QDs and QD/polymer hybrid structures in terms of their colloidal and optical properties at single molecule level. Combining different microscopy and spectroscopy techniques will enable simultaneous characterization of different properties such as morphology, optical and colloidal stability, etc...

In Chapter 4 the morphology and the nanomechanical properties of individual PNIPAM microgels as probed by atomic force microscopy, AFM, at different experimental 
conditions were discussed. The particles were imaged in air and in water at temperatures below and above the polymer volume-volume phase transition temperature, VPTT. The measured height and width values of the PNIPAM particles adsorbed at the silicon surface revealed that the particles undergo large changes in volume and characteristic dimensions as a function of experimental conditions. Decreased solvency of the particles at T $>$ VPTT as well as the changes in the interactions between the particles with the substrate were mainly responsible for the observed morphological changes. As probed by AFM-based nanoindentaion experiments, the related changes in the crosslink densities at the nanoparticles surface resulted form a combination of the chemical crosslinks and introduction of large amount of physical crosslinks above the lower critical solution temperature, LCTS, of PNIPAM. The results presented will serve as a reference for the studies of surface mechanical properties of hybrid polymer/nanoparticle materials.

In Chapter 5, a thermo-responsive metal/polymer/quantum dot platform based on PNIPAM brushes grafted from gold substrate using an iniferter-type polymerization, and QDs covalently attached to the PNIPAM chain ends was demonstrated. The EDC/NHS chemistry is shown to be efficient in coupling of $\mathrm{COOH}$ functionalized QDs to amineterminated PNIPAM brushes. Luminescence imaging and luminescence spectra measurements showed that upon increasing the temperature above the LCST of PNIPAM the QD emission was quenched. When decreasing the temperature below the LCTS, recovery of the luminescence could be observed. The results obtained were consistent with temperature modulated thickness of the PNIPAM brushes and quenching of the nanoparticles luminescence by the gold substrate. The Au/PNIPAM/QD system reported here may be explored in sensing and surface thermometry. The iniferter-type polymerization would also allow one to control the film thickness and improve the sensitivity and time response of the system.

Chapter 6 described an efficient method for the fabrication of water soluble, temperature-responsive QD/PNIPAM assemblies. A maleic anhydride-based polymer was used as backbone for grafting PNIPAM chains of three different molar masses. The QDs coated with the polymers were successfully transferred to water. The hybrid nanoparticles exhibited LCST behavior at temperature similar to those of free polymers. These nano-sized, temperature responsive hybrid particles hold a great promise towards realization of sensor devices with a broad range of applications.

In Chapter 7 the colloidal and optical properties of QD/PNIPAM hybrid materials at temperatures below and above the LCST of PNIPAM were characterized. Taken together, the 
FCS and TCSPC data provided strong evidence on the temperature-induced chain collapse of PNIPAM at T $>$ LCST. The proven colloidal and optical stability of QD/PNIPAM hybrids at T $>$ LCST of PNIPAM suggested that surface engineering of QDs with thermo-responsive PNIPAM chains enable realization of hybrid assemblies with switchable optical and colloidal properties as a function of temperature. These materials are also suitable for in vivo applications, where the temperature is readily higher than the LCST of PNIPAM.

In Chapter 8 applications of QDs in bioscience and medicine were reviewed with a special emphasis on the QD cytotoxicity. With the recent advances in nano-fabrication technologies it is very likely that the use of QD products will increase. Therefore, it is necessary to elucidate the potential adverse effects of these materials not only for the concerns about the human health and environmental protection, but also for the industry to make use of these promising materials in larger scales.

Given the potential benefits offered by QD technology, clarifying the mechanisms and sources of QD toxicity will help avoid problems that could be encountered due to misapplication of this technology. Only with this knowledge it will be possible to achieve a QD technology that is compatible with the social and ecologic systems, and that has the public support. 



\section{Samenvatting}

Quantum dots (QDs) krijgen steeds meer aandacht vanuit veel onderzoeksdisciplines wegens hun voordelen als fluorescente bakens, waaronder de nanoschaal grootte (overeenkomstig met de grootte van biomoleculen), de hoge quantum efficiëntie, hoge molaire uitdovingscoëfficienten, veelzijdigheid op het gebied van oppervlakte modificatie, brede excitatie spectra (voor meerkleuren imaging) en een nauwe emissieband en instelbare optische eigenschappen. Het fabriceren van $\mathrm{QD} /$ polymeer hybride nanosctructuren maakt de realisatie mogelijk van veel potentiële applicaties zoals opto-electronische apparaten, biologische sensoren, en fotonische structuren, omdat het insluiten van QDs in polymeer matrices niet enkel de controle over de optische en spectroscopische eigenschappen van de QDs mogelijk maakt, maar ook een sterke weerstand tegen chemische en fotonische degradatie mogelijk maakt. Het onderzoek dat is beschreven in dit proefschrift heeft als eerste doel de synthese en karakterisatie van $\mathrm{CdSe} / \mathrm{ZnS}$ core/shell QDs, en van temperatuurresponsieve polymeer matrices gemaakt van poly( $N$-isopropylacryl amide), PNIPAM, als dragers van QDs. Het tweede doel is de fabricatie van QD/PNIPAM structuren die potentieel gebruikt kunnen worden als meetinstrumen in de bio-nanotechnologie.

Hoofdstuk 1 is een algemere introductie van dit proefschrift. Hoofdstuk 2 geeft achtergrand informatie over de optische eigenschappen van QDs. Daarnaast worden de recente ontwikkelingen met betrekking tot het combineren van stimulusgevoelige polymeren met QDs via verschillende methodes, waaronder laag-voor-laag depositie, macromoleculaire grafting en in situ QD synthese in polymeer matrices bechouwd.

Hoofdstuk 3 beschrijft de basis van enkele microscopie en spectroscopie technieken die worden gebruikt voor de karakterisatie van QDs tot op het niveau van een enkel molecuul. Naast atomic force microscopie en confocale optische microscopie, worden gecombineerde microscopie technieken, die gebruikt zijn voor het besturderen van QDs en systemen die QDs bevatten uitgelegd. Onder de ultrasnelle spectroscopie technieken welke in dit hoofdstuk genoemd worden vallen onder andere time correlated single photon counting en fluorescence correlation spectroscopie. De microscopie en spectroscopie technieken die worden besproken zijn een nuttig gereedschap voor de karakterisatie van QDs en QD/polymeer hybride structuren op het gebied van hun colloïdale en optische eigenschappen op het niveau van een enkel molecuul. Het combineren van verschillende microscopie en 
spectroscopie technieken zal het simultaan karakteriseren van verschillende eigenschappen zoals morfologie, optische en colloïdale stabiliteit, etc. mogelijk maken.

In Hoofdstuk 4 worden de morfologie en de nanomechanische eigenschappen beschreven van individuele PNIPAM microgels, zoals onderzocht met de AFM onder verschillende experimentele condities toestanden. De deeltjes worden afgebeeld in lucht en in water bij temperaturen zowel onder als boven de polymeer VPTT. De gemeten hoogte en breedte waardes van de PNIPAM deeltjes welke geadsorbeerd zijn op het silicium oppervlak, onthullen dat de deeltjes een grote verandering in volume en karakteristieke dimensies ondergaan, welke afhankelijk is van de experimentele toestanden. Zowel een afgenomen oplosbaarheid van de deeltjes bij T $>$ VPTT als de veranderingen in de interacties tussen de deeltjes met het substraat zijn hoofdzakelijk verantwoordelijk voor de waargenomen morfologische veranderingen. De gerelateerde veranderingen in de crosslink dichtheden aan het oppervlak van de nanodeeltjes, zoals gemeten met op AFM gebaseerde nanoindentatie experimenten, komen voort uit een combinatie van de chemische crosslinks en vele geïntroduceerde fysische crosslink boven de LCTS van PNIPAM. De resultaten die worden gepresenteerd dienen als een referentie voor het bestuderen van mechanische eigenschappen van hybride polymeer/nanodeeltjes materialen.

In Hoofdstuk 5 wordt een thermoresponsief metaal/polymeer/QD platform gebaseerd op PNIPAM borsteltjes grafted op een goud substraat met gebruik van een iniferter-type polymerisatie, met QDs covalent gebonden aan het einde van de PNIPAM ketens, gedemonstreerd. Er wordt aangetoond dat de EDC/NHS chemie efficient is met betrekking tot het verbinden van $\mathrm{COOH}$ gefunctionaliseerde QDs aan amine-getermineerde PNIPAM borsteltjes. Luminescentie imaging en metingen van luminescentie spectra laten zien dat de QD emissie gequenched wordt als de temperatuur boven de LCST van PNIPAM komt. Als de temperatuur wordt verlaagd tot onder de LCTS, is herstel van de luminescentie waarneembaar. De verkregen resultaten zijn consistent met de temperatuur gemoduleerde dikte van de PNIPAM borsteltjes en uitdoving van de luminescentie van de nanodeeltjes door het gouden subtraat. Het Au/PNIPAM/QD systeem waar hier melding van wordt gemaakt biedt mogelijkheden voor oppervlakte thermometrie. De iniferter-type polymerisatie stelt men in staat om de dikte van de film, en hiermee de gevoeligheid en tijdsrespons van het systeem, te beïnvloeden.

Hoofdstuk 6 beschrijft een efficiënte methode voor de fabricatie van, in water oplosbare en temperatuur gevoelige, QD/PNIPAM structuren. Een maleic anhydridegebaseerd polymeer wordt gebruikt als backbone voor het graften van PNIPAM ketens met 
drie verschillende molaire massas. De met polymeren gecoated QDs zijn succesvol overgebracht in water. De hybride nanodeeltjes tonen LCST gedrag bij temperaturen vergelijkbaar met die van vrije polymeren. Deze nanogrootte, temperatuur responsieve hybride deeltjes zijn veelbelovend voor de realisatie van sensors met een grote verscheidenheid aan applicaties.

In Hoofdstuk 7 worden de colloïdale en optische eigenschappen van QD/PNIPAM hybride materialen gekarakteriseerd bij temperaturen boven en onder de LCST van PNIPAM. Samen leveren de FCS en TCSPC een sterk bewijs voor de temperatuur geïnduceerde ineenstorting van PNIPAM bij T>LCST. Verschillende graden van QD confinement met polymeer backbones, welke een verschillend aantal PNIPAM ketens van verschillende lengte bevatten, resulteren in hybride materialen met verschillende mate van responsiviteit met betrekking tot temperatuur. Deze materialen zijn tevens geschikt voor in vivo toepassingen, waar de temperatuur doorgaans hoger is dan de LCST van PNIPAM.

In Hoofdstuk 8 worden toepassingen van QDs in de biowetenschap en geneeskunde belicht met een nadruk op de cytotoxiciteit van QDs. Met de recente ontwikkelingen in nanofabricage technologieën is het zeer waarschijnlijk dat het gebruik van QD producten toe zal nemen. Daarom is het noodzakelijk om een licht te schijnen op de mogelijk nadelige effecten van deze materialen. Niet enkel wegens de zorgen met betrekking tot gezondheid en milieubescherming, maar tevens voor de industrie, opdat zij op grotere schaal gebruik kan maken van deze veelbelovende materialen.

Gegeven de potentiële voordelen welke QD technologie brengt, zal het ophelderen van de mechanismen en de oorzaken van QD toxiciteit helpen om problemen te voorkomen welke tegengekomen kunnen worden bij de verkeerde toepassing van deze technologie. Enkel met deze kennis zal het mogelijk zijn om een QD technologie te bereiken welke verenigbaar is met de sociale en ecologische systemen, en de steun van het publiek heeft. 



\section{Pêşgotîn bi Kurtayî}

Niqtayên Kuantumê, (NK) probên fuloresan bi dîmenên nanometerîn. Ji ber xisûseten optîkî qu dikarin werin sazkirin, niqtayên kuantumê li lekolînên zanîyarî alakayêk mezin dikşînin. Ji ber ku niqtayên kuantumê di zanîstê bîyolojî were baş karkirin rûyên wan ger werîn bi polîmeran modîfe kirin. Bi alîkarîyên polîmeran mirov dikarê xisûseten optîkî ye niqtayên kuantumê kontol bike, li alîyên din mirov dikarê niqtayên kuantumê ji xirabbûnên kîmyayî biparêze. Armanca vê têzê niqtayên kuantumê bi polîmerek (N-izopropilakrilamid), PNIPAM, ku hesasê germahî yê, yekbike û bi vê yêkê çêkirina cîhazên hîbrît bi sensor ku xwedîyê dîmenên nanometerîn.

Beşa 1, li vê têzê bi babêtê giştî dirîne. Beşa 2, xisûseten optîkî yê niqtayên kuantumê li ser bingêha teorîk û tetbiqên ku di lîteratûrda hatîn nîşandan ihtîva dike. Beşa 3, behsa hin metodên mikroskopî û spektroskopî ku ji bo karekterîzasyonê niqtayên kuantumê dike. Beşa 4, behsa lekolîna bi alîkarîyê mikroskopek atomî li ser perçêyen PNIPAM ku hesasê germahîyêne û dîmêne van 500 nanometerê dike. Beşa 5, behsa çekirin û tetbîqkırına sensorêkî germahiyê ku ji metal, polîmerên hesasê germahîyê û ji niqtayên kuantumê hatîye avakirin dike. Beşa 6 , behsa sentez û tatbîqa block co-polîmer dike ku ji niqtayên kuantumêre îmkana helîna van li awê dide û van hesasê germahîye dike. Beşa 7, behsa karekterîzasyona optîk bi detayî yen sentezên ku li beşa 6 da hatin kirin dike. Beşa 8 , behsa potansîyale bandûra tênişt yen niqtayên kuantumê li ser sîstemên bîyolojî dike. Dîsa li vir behsa çend metodên ku van bi bîyo ahengdar dike tekirin. 



\section{Acknowledgements}

There are certain events in life that change the entire direction of the rest of the 'road'. If I was asked to name, coming to Enschede to pursue my $\mathrm{Ph} \mathrm{D}$ degree would definitely be one of those. By giving me the opportunity to work on this project, Prof. G. Julius Vancso definitely contributed the most to the realization of this 'new-beginning' in my life. Dear Julius, thank you very much for accepting me as a member of MTP. I have really enjoyed all the scientific and intellectual discussions we had, which had occasionally been quite harsh. I have learned a lot from your expertise. Prof. Jennifer L. Herek, as my promoter, also helped me a lot with her expertise in this project. Dear Jennifer, I really enjoyed all the scientific and social activities we had with the OS group. Unfortunately I had the opportunity to work with you only towards the end of my project. Thank you for your great contributions to this project within such a short period of time.

I would like to thank Dr. Nikodem Tomczak, the master Yoda, my daily supervisor. Dear Nikodem, I really appreciate your efforts to supervise me from the other side of the world. I really enjoyed our e-discussions. Working with you in Singapore for a couple of months was a great opportunity. Dr. Dominik Janczewski also helped me a lot during my stay in Singapore. Thank you very much Dominik. I would like to thank Prof. Ming-Yong Han (IMRE, Singapore) for the collaborations we had.

This work would not be possible without the financial support of $\mathrm{MESA}^{+}$Institute for Nanotechnology. Working in such an interdisciplinary project, I had the opportunity to collaborate with other groups at University of Twente. I would like to thank Prof. Vinod Subramaniam (BPE) for kindly agreeing to collaborate. During this collaboration I had the opportunity to work with Dr. Christian Blum and Dr. Yanina Cesa. Dear Christian and Yanina, I would like to thank you for our fruitful discussions as well as your technical assistance with the measurements.

The work presented in this thesis could not be realized without the help of many colleagues and friends. I'd like to thank Dr. Aliakbar Jafarpour for his contributions to the seventh Chapter of this thesis. I'd also like to thank Eric Theodore Cartman, the coolest person I've seen (after Vali Zakaee), for all his support. Many thanks to the rest of Optical 
Sciences group, especially to the female members Leila, Di and Jincy, who are the living proofs of the fact that physics is not a 'man' business. I really appreciate help of Alexander in proofreading of this thesis and translating the summary into Dutch. Many thanks to Dr. Herman Offerhaus, Martin, Dan, Bob, Kasper, Erik, Erwin and Robert for creating a nice working environment at the $10^{\text {th }}$ floor of Hogekamp. Jeroen, you were our savior in the lab. Frans, I really appreciate your dedication to doing sports (even at minus 10 degrees). Thank you guys for all the technical support in the lab. Karen, thank you very much for your help with the administrative and financial issues. I promise that I'll keep the train tickets for reimbursement.

During my $\mathrm{Ph} \mathrm{D}$, I really enjoyed the nice working atmosphere in Langezijds, which was made possible by the members of MTP. I enjoyed discovering Netherlands and drinking sessions with Eugenia, Denis and Eddy when I first arrived here. Guys, all of you are scattered around the world now and apparently I'll be the last one to leave Enschede. I would like to thank Janet (my dear friend with the mother attitude) for all the moments we spent together. Otel odalarinda sigara icip cildinin gozeneklerini kapamama ragmen bana katlanabildigin icin tesekkurler $(-)$. Ayrica Kris gibi sahane bi hatuna ablalik yaptigin icin de sagol ;). Mine (welcome to Enschede!), Edit, Joost (the joker), Qi (papa), Yujie (mama), Jing (mamma mia), Gabriella (oh lala), Wilma, Anika, XiaoFeng, Mathijs, and all the students of MTP, thank you for being great colleagues and friends! Maisha and Gervin, thank you very much for your contributions to my work in your Bachelor's projects. I'd also like to thank the staff members of MTP; Peter, Mark and Clemens. Clemens, I think Denis was right when he was saying that you would repair a spaceship with a screwdriver and a tape. Genevieve, thank you for having such great spirit all the time. I always appreciated your high mood.

Looking back to when I started my $\mathrm{Ph} \mathrm{D}$, I realize that I'm really missing many colleagues/friends who used to work at MTP. Szczepan, Holger, Thomas, Davide, Monique, In Yee, Marina \& Miguel (M\&M), Melba, Cynthia, Nina, Ewa and many others. It was really great to work with you.

During the 2 months I spent in Singapore I made very nice friends. I'd like to thank Ani, Sagar, Pur, Aiden, Nicky, Nguyen, Adit, Tom, Soubhik and all the 'SIC' residents, who made those two months unforgettable for me. 
Speaking of residents, I should also mention the members of 'the residents' Edit, Berk, Cem and Pinar for the nice time we spend together with the company of port with jazz, or palinka with movie. Guys, we should design that table sometime!

Jacob, the grandpa, thank you very much for all the lunch meetings we had, where you had to listen to me complaining for hours. I always enjoyed your company and appreciated your advices. I'd also like to thank Bilge, Jealemy, Stefano (why am see yeay) Francesca, Albert, Kim, Pelagia, Erhan, Arzu gizim, Zeynep, Aytac, Banu, Oktay, Duygu, Engin, Hasan, Selim, Feridun, Gregory, Chaitanya, Mukund, Can, Elif, Siggi, XingYi and many other friends that I can not recall now for making Enschede a fun place. Ankara 100. Yil atmosferini Utrecht'e tasiyan Rasim ve Ozlem’e de ayrica tesekkurler.

I'd like to thank again Edit Kutnyanszky and Alexander van Rhijn for accepting to be my paranimfen.

Without the constant and unconditional support of my wonderful parents Rabiye and Dursun, my sister Ozge and my brother Cem, I would not be the person who I am now. Annecigim, babacigim, baciconam ve abbasiconam, size ne kadar tesekkur etsem yine de eksik kalir bi’yerlerde bi’seyler. Bana verdikleriniz icin, inandiginiz ve guvendiginiz icin cok tesekkur ederim. Sizi cok seviyorum. Iyi ki varsiniz. Spas!

And finally, Vypiem za Rodinu!

I wish all the best for all of you.

Oya, 



\section{About the Author}

Oya Tagit was born in 21st May, 1981 in Bursa, Turkey. She obtained her bachelor degree of Biology from the Middle East Technical University (METU) in the year of 2003. She continued her master education in the same university and joined the group of Biomaterials and Tissue Engineering headed by Prof. Dr. Vasif Hasirci at METU. She received her master degree of Biotechnology in June 2005, with the research project entitled "Development and analysis of controlled release polymeric rods containing vancomycin". In October 2005, she started her PhD under the supervision of Prof. Dr. G. Julius Vancso and Prof. Dr. Jennifer L. Herek in the Material Science and Technology of Polymers (MTP) and Optical Sciences (OS) groups at the University of Twente, on the development and characterization of stimuli-responsive polymer/quantum dot hybrid platforms with potential applications in bio-nanotechnology. The results of this research work are described in this thesis. 